MAGAZIN FÜR STUDIERENDE UND SOZIOLOGIEINTERESSIERTE

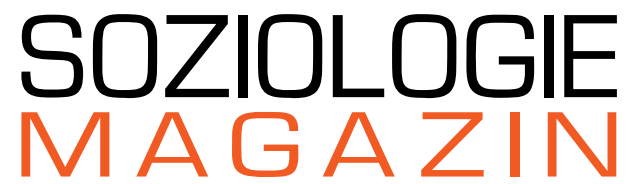

Publizieren statt archivieren
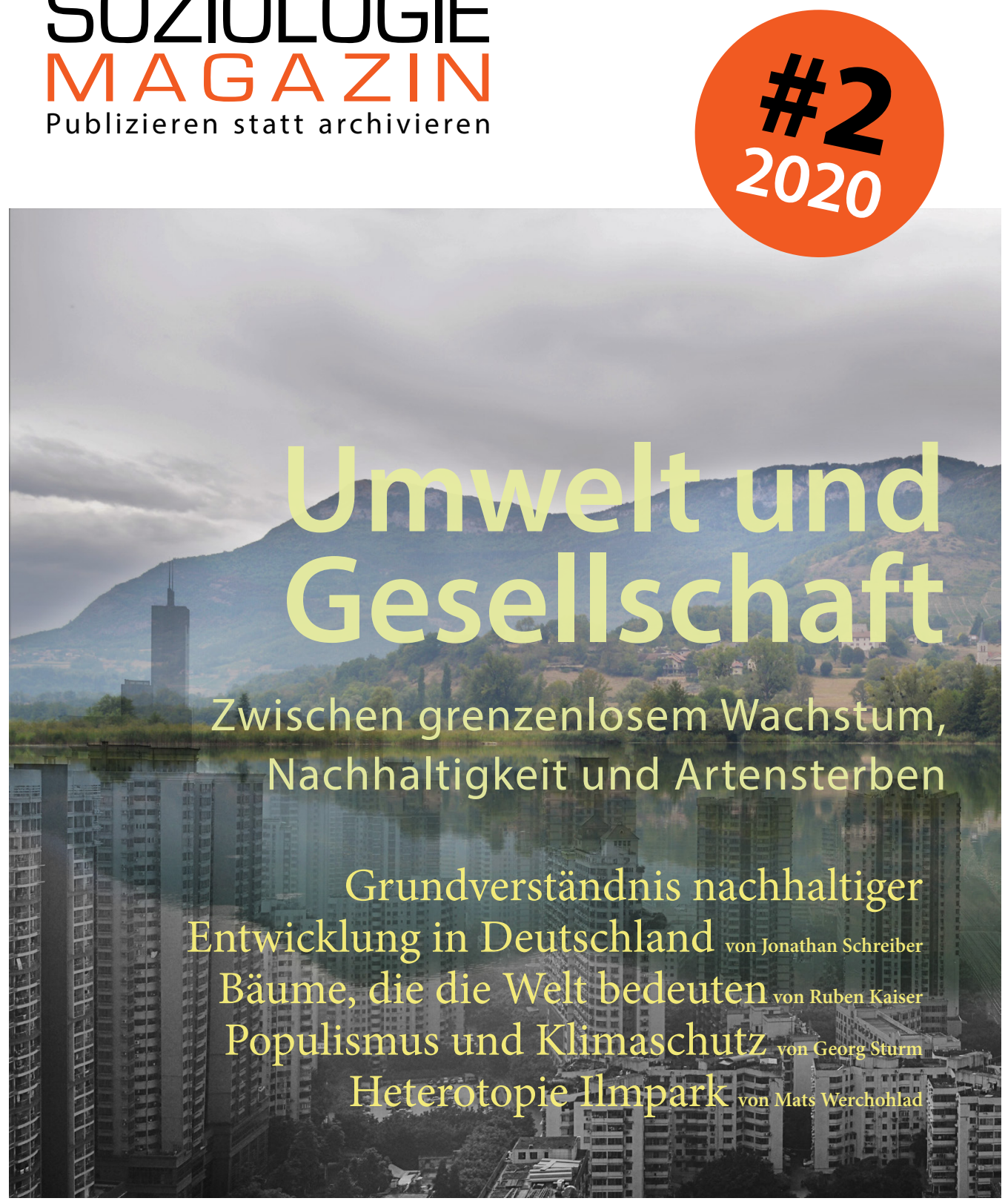

Außerdem: Expert*inneninterviews | Ausgewählte Fachliteratur | Konferenz- und Tagungstermine 2021 


\section{Zum Verhältnis von}

Mensch, Umwelt und Natur

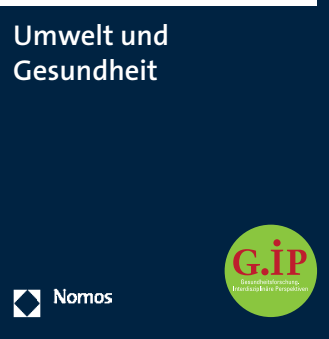

\section{Umwelt und Gesundheit}

Herausgegeben von PD Dr. Jens Soentgen,

Prof. Dr. Ulrich M. Gassner, Dr. Julia von Hayek und Prof. Dr. Alexandra Manzei

2020, 384 S., brosch., 79,-€

ISBN 978-3-8487-5511-0

(Gesundheitsforschung. Interdisziplinäre Perspektiven, $B d$. 2)

Über 20\% der jährlichen Todesfälle weltweit werden mit der Umwelt in Verbindung gebracht. Zugleich wandelt sich die Umwelt durch Klimawandel, Bevölkerungswachstum und Globalisierung. ExpertInnen aus zahlreichen Disziplinen diskutieren hierzu die neuesten Forschungsergebnisse im zweiten Band der interdisziplinären Schriftenreihe.

\section{Die Paradoxie der Ökologie}

Mit einem Vorwort von Armin Nassehi

Von Dr. Magdalena Göbl

2019, 289 S., brosch., 54,-€

ISBN 978-3-8487-5979-8

(Umweltsoziologie, Bd. 7)

Die Arbeit liefert eine systemtheoretisch-empirische Auseinandersetzung mit der Fragestellung, ob und wie sich eine moderne Gesellschaft aufökologische Herausforderungen einstellen kann und wie diese Fragen gesellschaftlich diskutiert werden, beziehungsweise diskutiert werden können. 


\section{Editorial}

\section{Umwelt und Gesellschaft. Zwischen grenzenlosem Wachstum, Nachhaltigkeit und Artensterben}

Die Umweltsoziologie ist im deutschsprachigen Raum eine weniger institutionalisierte Forschungsrichtung - vor allem ist sie aber ein interdisziplinäres Unterfangen. Für die Umweltsoziologie bedeutet dies zum einen Vermittlungsarbeit, schon bei der Formulierung von Problemen, der Entwicklung von Fragen, der Konzeption und Durchführung von Projekten. Zum anderen kommen ihr im Zusammenspiel mit den anderen Disziplinen wie den Naturwissenschaften die Aufgaben zu, Prozesse zu spiegeln, Haltungen zu befragen, vermeintliche Wahrheiten skeptisch zu begegnen, scheinbar Faktisches nicht unhinterfragt zu lassen.

Die öffentliche Aufmerksamkeit, die auf dem Thema Klimaschutz seit den ersten weltweiten Klimaprotesten 2019 und Greta Thunbergs „Skolstrejk för klimatet“ liegt, rückt auch die umweltsoziologische Forschung ins öffentliche Blickfeld. Die Bedeutung der Umweltsoziologie in der anwendungsnahen Forschung und in außeruniversitären Kontexten wächst beständig. Hieraus ergeben sich neben Fragen insbesondere auch Chancen für das vergleichsweise junge Forschungsfeld, welches sich in den 1990er Jahren auch im deutschsprachigen Raum etablierte.

Welche Rolle der Umweltsoziologie innerhalb eines interdisziplinären Feldes zukommt, mit welchen Herausforderungen Umweltsoziolog*innen in der Zusammenarbeit mit anderen Disziplinen umgehen müssen und inwiefern die Umweltsoziologie eine politische Wissenschaft ist, darüber hat Cathrin Mund für dieses Heft mit Umweltsoziologe Matthias Großgesprochen. Wenngleich das Entstehen der Umweltsoziologie mit den Umweltdiskursen der 1970er eng verbunden ist, warnt Groß davor, sich mit aktuellen Diskursen der Klimabewegung gemein zu machen oder sich in ihren Dienst zu stellen. Vielmehr sieht er für die Soziologie die Möglichkeit, im Sinne einer kritischen Wissenschaft für Begrifflichkeiten zu sensibilisieren, sich mit Phänomenen wie einer neuen Wissenschaftsbegeisterung auseinanderzusetzen und Argumentationen von Klima- und Umweltbewegungen auf den Prüfstand zu stellen. 
Wie Anna-Katharina Brenner, PhDKandidatin der Doktoratsschule Transition to Sustainability, innerhalb eines interund transdisziplinäre Forschungsfeldes im Bereich der nachhaltigen Entwicklung arbeitet und Themen entwickelt, dazu hat Andreas Schulz die Nachwuchswissenschaftlerin befragt. Neben Einblicken in ihre Forschung, die sich an den Schnittstellen von Politischer Ökologie, Kritischer Stadtforschung und Sozialer Ökologie positioniert, gibt Brenner zu bedenken, dass technologische Neuerungen nicht ausreichen werden, um eine ökologisch nachhaltige Zukunft zu realisieren.

Auf Basis einer repräsentativen Onlinebefragung nähert sich Jonathan Schreiber in seinem Beitrag „Grundverständnis nachhaltiger Entwicklung in Deutschland. Ökologische Modernisierung oder Postwachstum "der Frage an, welche Einstellungen zu ökologischer Modernisierung und Postwachstum in der deutschen Gesellschaft vorherrschen.

Mit einem Symbol der deutschen Klimabewegung, dem Hambacher Forst, setzt sich Ruben Kaiser auseinander: In seinem Beitrag „Bäume, die die Welt bedeuten "zeigt er, wie Protestbewegungen wissenschaftliche Erkenntnisse zum Klimawandel in klimapolitisches Wissen überführen und über soziale Praktiken ein neues Umweltbewusstsein herstellen. Am Beispiel der Waldspaziergänge im Hambacher Forst expliziert er, wie „niedrigschwellige integrative Versammlungsformate "der Erar- beitung von politischem und zivilgesellschaftlichem Wissen dienen und zur Formierung von Klimaprotesten beitragen.

Wie wiederum autoritär-populistische Parteien das Thema Klimaschutz für sich nutzen, um populistisches Protestpotential zu erzeugen, untersucht Georg Sturm auf Basis einer Analyse von AfD-Pressemitteilungen. Er zeigt in "Populismus und Klimaschutz", wie die rechtspopulistische Partei bei den Themen Klimaschutz und Klimawandel ökonomische Verlusts- und Abstiegsängste von Wähler*innen anspricht und zugleich Gefühle von kultureller und politischer Entfremdung adressiert.

In unserem Perspektiventeil zeigt Mats Werchohlad mit seinem Essay „Heterotopie Ilmpark", wie der Ilmpark in Weimar zu einem Forschungs-, Erlebnis- und Verhandlungsraum für die kollektive Suche nach einer neuen Naturästhetik werden soll. Der Autor versteht den Park als Abbild einer konfliktreichen Gegenwart, bedingt durch widerstrebende Akteur ${ }^{\star}$ inneninteressen und klimatische Veränderungen, und bietet der Soziologie an, sich aus ebenjener Perspektive mit dem Konzept des Raumes auseinanderzusetzen.

Einen Überblick über zentrale und aktuelle Werke der umweltsoziologischen Forschung hat Luisa Bischoff zusammengetragen. Tonka Radisch hat eine Reihe digitaler und physischer Tagungen und Veranstaltungen recherchiert. 
Während diese Ausgabe entstand, hat der soziologiemagazin e.V. seinen ersten Sammelband veröffentlicht. Die beiden Herausgeber*innen und Redakteur*innen Andreas Schulz und Tamara Schwertel haben hierfür neben Forschungs-, Reflexions- und Perspektivenbeiträgen aus der Redaktion auch Beiträge aus einem erweiterten Bekannt*innenkreis für das Buchprojekt „Der lange Sommer der Flucht - 2015 und die Jahre danach" gewinnen können. Der Band erschien im August im Verlag Barbara Budrich und vereint Perspektiven aus Soziologie, Kommunikationswissenschaft, Kultur- und Sozialanthropologie sowie der Sozialpsychologie. Geplant ist weiterhin eine zweite Ausgabe der Soziologischen Fragmente. Diese schließt thematisch an unsere im April gestartete Blogreihe Soziologische Impulse während COVID-19 an.

Wir danken allen Beitragenden für Ihre Texte, Ihre Impulse und das konstruktive Wirken an dieser Ausgabe. Allen Leser ${ }^{*}$ innen wünschen wir eine anregende Lektüre und bedanken uns herzlich für das Interesse - bleibt uns gewogen, bleibt kritisch.

Stellvertretend für die Redaktion des Soziologiemagazins, September 2020

\section{Cathrin Mund und Andreas Schulz}

Das Editorial wurde von Tanja Strukelj lektoriert.

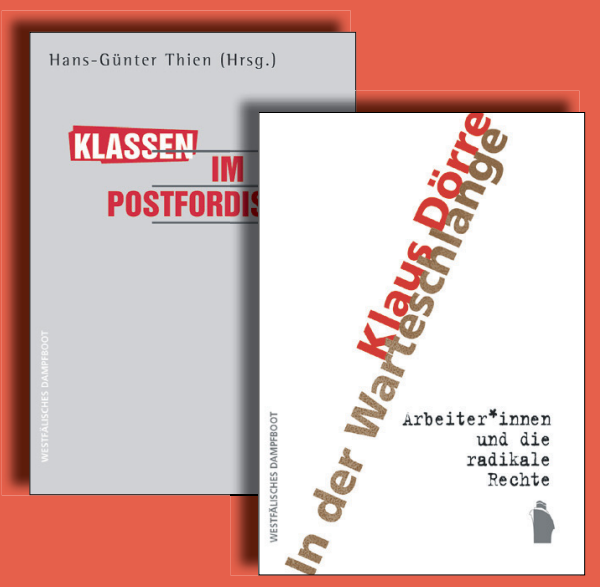

Klaus Dörre

erscheint in Kürze

\section{In der Warteschlange}

Arbeiter*innen und die radikale Rechte

2020 - ca. 320 Seiten - ca. 30,00 € - ISBN 978-3-89691-048-6

Hans-Günter Thien (Hrsg.)

3. Auflage

\section{Klassen im Postfordismus}

2020 - 381 Seiten - 35,00€ - ISBN 978-3-89691-781-2

Der ... Sammelband bietet einen gerafften Überblick über die aktuelle Debatte über Klassen vor allem im deutschsprachigen Raum, der ältere Beiträge, eher konventionelle Ansätze und innovative Vorstöße - sowohl in konzeptioneller wie empirischer Hinsicht - vereint. Dieter Boris - $Z$. 


\section{Das neue Leben nach der Krise}

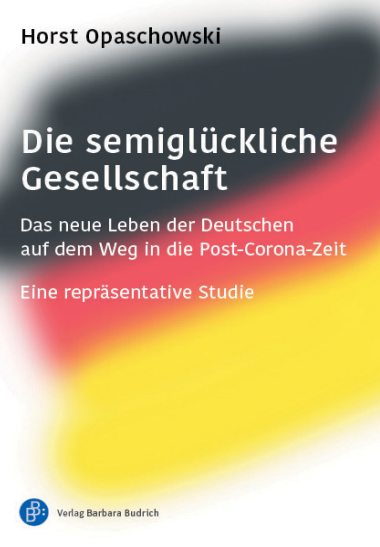

\section{Horst Opaschowski}

Die semiglückliche Gesellschaft. Das neue Leben der Deutschen auf dem Weg in die Post-Corona-Zeit. Eine repräsentative Studie $166 \mathrm{~S}$. | Gebunden mit Schutzumschlag 19,90 EUR (D) | 20,50 EUR (A)

ISBN 978-3-8474-2466-6

Prof. Dr. Horst Opaschowski ist Zukunftswissenschaftler und Berater für Wirtschaft und Politik. Er gilt als Begründer der Zukunftsforschung in Deutschland.

Wie sehen die Deutschen angesichts der Corona-Pandemie in die Zukunft? Diese erste repräsentative Studie über das neue Leben der Deutschen vor und während der Corona-Krise zeigt: Viele Menschen wurden ärmer, aber nicht unglücklicher. Ihr Wohlstandsdenken veränderte sich, und Gesundheit wurde so wertvoll wie Geld. Zeitwohlstand und Beziehungsreichtum kamen als neue Lebensqualitäten hinzu, und auch der Staat strahlte soziale Wärme aus. Die Zuversicht wächst also wieder - auch in unsicheren Zeiten.

Die repräsentative Deutschlandstudie umfasst den Zeitraum von der Prä-Corona-Zeit im Januar 2020 über die Corona-Krise im März 2020 bis zu den Corona-Lockerungen ab Juli 2020. 


\section{\# 2 / 2020}

Editorial

Umwelt und Gesellschaft. Zwischen grenzenlosem Wachstum, Nachhaltigkeit und Artensterben. von Cathrin Mund \& Andreas Schulz

\section{Interviews}

„Wer wie die Umweltsoziologie in interdisziplinären Kontexten arbeitet, lernt, sich nicht an eine soziologische Theorie oder einen methodischen Zugang zu klammern.“

Ein Experteninterview mit Matthias Groß | geführt von Cathrin Mund

„Es braucht einen tiefgreifenden sozial-ökologischen gesellschaftlichen Wandel“

Ein Expertininterview mit Anna-Katharina Brenner | geführt von Andreas Schulz

\section{Schwerpunkt}

Grundverständnis nachhaltiger Entwicklung in Deutschland

Ökologische Modernisierung oder Postwachstum | von Jonathan Schreiber

Bäume, die die Welt bedeuten

Der Hambacher Forst als Symbol der deutschen Klimabewegung | von Ruben Kaiser

Populismus und Klimaschutz

Der AfD-Klimadiskurs | von Georg Sturm

\section{Perspektiven}

Heterotopie Ilmpark.

Raum zur Suche nach einer neuen Umweltästhetik | von Mats Werchohlad

Literatur zum Thema

\section{Aus der Redaktion}

Tagungen und Termine

Redaktionsteam und Danksagung.

Impressum 

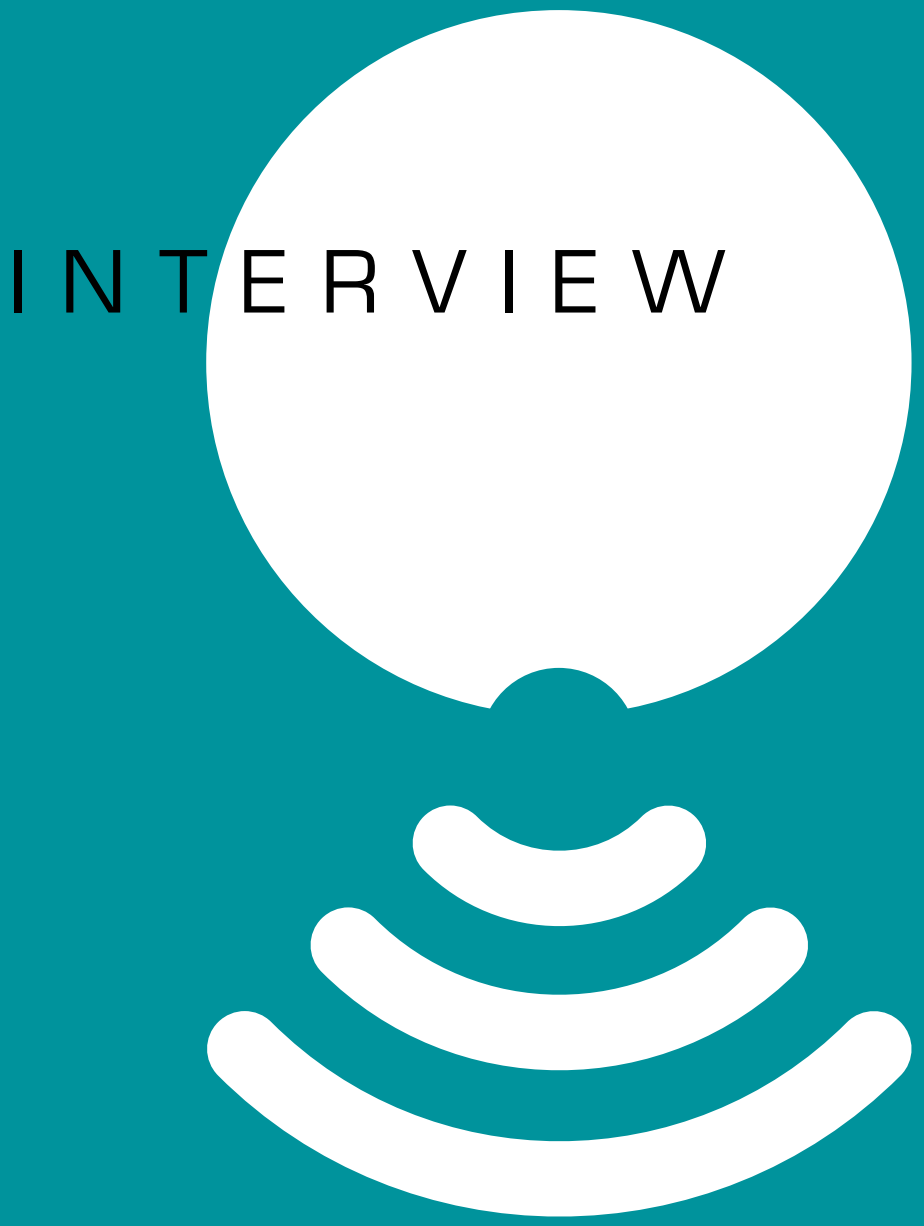


\section{"Wer wie die Umweltsoziologie in interdisziplinären Kontexten arbeitet, lernt, sich nicht an eine soziologische Theorie oder einen methodischen Zugang zu klammern."}

\section{Ein Experteninterview mit Matthias Groß}

von Cathrin Mund

SozMag: Natur, Umwelt, Klima - sehr geehrter Matthias Groß, lassen Sie uns mit einer Verständnisfrage einsteigen. Mit welchem Begriff von Umwelt arbeiten Sie? Welche Verständnisse von Umwelt herrschen aktuell in der soziologischen Forschung vor?

Groß: Nun ja, da muss man vielleicht ein bisschen ausholen und erläutern, wo denn die Umweltsoziologie und grundlegende soziologische Verständnisse vom Umgang mit Außersozialem, also Natur, Ökologie oder allgemein den materiellen Dingen um uns herum herkommen. Der Begriff Environmental Sociology wurde, wenn auch nur vereinzelt, zuerst in der Architekturtheorie in den 1960er Jahren benutzt. Dabei ging es um die Bedeutung der Wohnumwelt und die Wirkung dieser auf das menschliche Befinden. Vor dem Hintergrund der Umweltbewegung war dann die nordamerikanische Environmental Sociology Mitte der 1970er Jahre mit nicht weniger gestartet als dem Plan, die Soziologie allgemein einem Paradigmenwechsel zu unterziehen. Die Soziologie sollte grundlegend umgebaut werden, weg von einer reinen Sozialwissenschaft. Das war damals durchaus schon im Sinne heutiger Diskussionen um Posthumanismus oder 
den Neuen Materialismus zu verstehen gewesen. Durch die Umweltbewegung wurde das Bewusstsein über die Abhängigkeit gesellschaftlicher Entwicklungen von ökologischen Prozessen auch in Teilen der Soziologie immer deutlicher, was dann zu einer eigenen Subdisziplin führte. Die Debatten darüber, wie man eine solche Disziplin nennen könnte, in der neben sozialen auch ökologische Variablen Einzug in Modelle und Analysen der Soziologie finden dürfen, schließt an Ihre Frage nach den Verständnissen von Umwelten an. Umwelt oder Environment bezeichnete in der Soziologie lange Zeit das, was mit der Umweltbewegung als die vom Menschen veränderte Umwelt, also die die Gesellschaft umgebende und einbettende materielle lebende Welt aufgefasst wurde. Es gab spätestens seit den 1980er Jahren hitzige Diskussionen darüber, ob man nicht eher von "Ökologischer Soziologie“ oder "Natursoziologie“ oder eben auch mit Verweis auf die Identität der Disziplin eher von der „Soziologie der Umwelt“ oder der „Soziologie der Natur" sprechen sollte. Da der Umweltbegriff im Alltag oft synonym mit Natur oder auch Ökologie verwendet wird, hat man sich dann auf Environmental Sociology bzw. im Deutschen auf „Umweltsoziologie“ geeinigt. Es gibt zwar heute immer wieder Einzelstimmen, die fordern, man solle den Begriff erweitern, um Begriffe wie Nachhaltigkeit, Resilienz oder den Begriff des Klimas. Im internationalen Kontext habe ich das allerdings noch nicht gehört. Im Gegenteil, die Kämpfe um die Begrifflichkeit haben sich gelegt.

SozMag: Sie sprechen die synonyme Verwendung des Begriffes Umwelt an. Welche Möglichkeiten eröffnet solch ein weiter Begriff für die umweltsoziologische Forschung?

Groß: Der Begriff der Umwelt, in all seiner Breite, lässt ihn zum einen schnell unscharf werden, zum anderen verweist er aber recht eindeutig auf etwas außerhalb der sozialen Welt, mit dem Gesellschaft in kausalen Wechselwirkungen steht. In Deutschland war die Wahl eines weiten Begriffes durchaus im Sinne einer neuen Soziologie, die eben nicht nur Soziologie der Umwelt sein wollte. Sondern mehr als das, eben eine „umweltliche“ oder „umweltbedingte“ Soziologie. Im Deutschen kommt das, anders als im Englischen mit dem Wort „environmental“, leider nicht so elegant rüber. Es ist dabei auch interessant zu bemerken, dass die Sektion Umweltsoziologie der Deutschen Gesellschaft für Soziologie (DGS) bei ihrer Gründung 1996 durch prominente Vertreter wie Ulrich Beck und Karl-Werner Brand zuerst Sektion „Soziologie und Ökologie“ getauft wurde. Damit wollte man genau diesem Aspekt des Paradigmenwechsels innerhalb der Soziologie gerecht werden. Nun ja, man ist dann nach etwa zehn Jahren, nicht zuletzt auch, um sich an das international etablierte Label Environmental Sociology anzulehnen, auf „Umweltsoziologie“ umgeschwenkt. 


\section{Matthias Groß}

Matthias Groß studierte Soziologie in Bielefeld und Arcata (Kalifornien, USA) und promovierte 2001 am Institut für Wissenschafts- und Technikforschung (IWT) der Universität Bielefeld. 2008 erfolgte die Habilitation in Soziologie an der Martin-Luther-Universität Halle. Er war DAAD-Fellow an der University of Wisconsin in Madison und lehrte unter anderem an Universitäten in Chicago, Halle, Karlsruhe und Leipzig. Seit 2013 ist er Professor für Umweltsoziologie an der

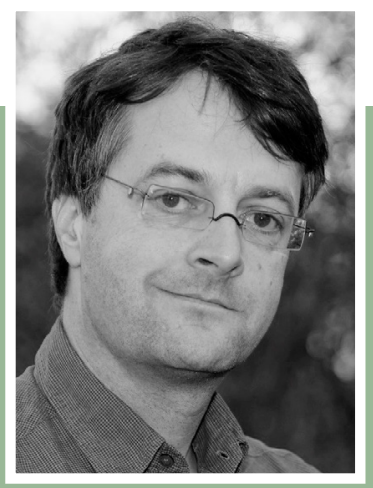
Friedrich-Schiller-Universität Jena sowie dem Helmholtz-Zentrum für Umweltforschung (UFZ) in Leipzig, wo er das Department Stadt- und Umweltsoziologie leitet. Er ist Mitbegründer und Herausgeber der Zeitschrift Nature + Culture sowie Coordinating Editor von Restoration Ecology.

Website: www.ufz.de/index.php?de=38287

Dass die Verständnisse von Umwelt in der soziologischen Forschung heute sehr weit gefasst sind, ermöglicht eine gute Anschlussfähigkeit - auch an andere Disziplinen - sowie die Unabhängigkeit von Trendbegriffen wie etwa Nachhaltigkeit und in gewisser Weise auch Natur und Ökologie. Nachteil ist die Tatsache, dass man je nach Forschungsfeld erst einmal Definitionsarbeit leisten muss. Aber das sind wir ja in unserer Zunft gewohnt. Von daher kann die Umweltsoziologie mit dieser Breite an Umweltverständnissen sehr gut leben, so wie auch die Kultursoziologie mit einem Potpourri an Kulturbegriffen leben kann oder auch die Technik- oder Wissenssoziologie verschiedene Verständnisse ihres Gegenstandes vorweisen können.
SozMag: Die Umweltsoziologie ist in Deutschland vergleichsweise jung: Erst im Laufe der 1990er Jahre entstanden Arbeiten, die sich mit den Herausforderungen der Naturthematik befassten und breitere soziologische Diskussionen einleiteten. Wie sind Sie zur Umweltsoziologie gekommen?

Groß: Das stimmt. Die Umweltsoziologie ist, fast konträr zur Entwicklung in Nordamerika, Skandinavien, den Niederlanden, Großbritannien, vielen asiatischen Ländern, ja im Grunde dem Rest der Welt, bis heute an deutschen Universitäten nur schwach institutionalisiert. Und das, obwohl es bereits in den späten 1970er Jahren einige Vertreter ${ }^{*}$ innen gab, die man einer Umweltsoziologie hätte zurechnen können, 
wie zum Beispiel verschiedene Autor*innen, die zur Anti-AKW-Bewegung oder frühen Öko-Bewegung gearbeitet haben. Auch die beiden Buchklassiker von 1986, Ulrich Becks Risikogesellschaft und Niklas Luhmanns Ökologische Kommunikation boten in gewisser Weise Steilvorlagen - und interessanterweise wird Beck international heute oft als Umweltsoziologe gesehen. Richtig los ging es aber in Deutschland erst in den 1990er Jahren.

Wie bin ich zur Umweltsoziologie gekommen? Nun, ich komme eigentlich aus der Wissenschafts- und Technikforschung. Interessant war dann, dass sich bestimme Stränge der Wissenschaftssoziologie, der Science and Technology Studies (STS), aber auch die Techniksoziologie in mancherlei Hinsicht analog zur Umweltsoziologie mit der Bedeutung der materiellen Umwelt in und für die Analyse gesellschaftlicher Prozesse befasst hat. Man denke nur an Hans Lindes Buch Sachdominanz in Sozialstrukturen, bereits von 1972, also lange vor Akteur-Netzwerk-Theorie und Co. Da ich als Jugendlicher und junger Student, wie viele in meiner Generation, stark von der Ökologiethematik beeinflusst und bewegt war, war es kein Wunder, dass ich die "natürliche" Umwelt auch soziologisch begreifen wollte. Aber erst durch ein Auslandsjahr in Kalifornien 1995/96 erfuhr ich von der Existenz einer Environmental Sociology und ich merkte, dass das, was da diskutiert wurde, zumindest konzeptuell von der Wissenschafts- und
Technikforschung nicht weit entfernt ist. Dass es auch eine aktive Community von Umweltsoziolog*innen in Deutschland gab, davon hörte ich erst ein paar Jahre später. Das muss so um 2000 herum gewesen sein.

SozMag: An der Friedrich-Schiller-Universität Jena sind Sie seit 2013 Professor für Umweltsoziologie. Am Helmholtz-Zentrum für Umweltforschung (UFZ) in Leipzig leiten Sie das Department Stadt- und Umweltsoziologie. Skizzieren Sie uns bitte einmal Ihre aktuellen Aufgaben-und Forschungsbereiche.

Groß: Meine Professur ist eine gemeinsame Berufung zwischen einer Universität und einem außeruniversitären Forschungszentrum der Helmholtz-Gemeinschaft. Neben der Lehre und der Betreuung von Studierenden und Qualifikationsarbeiten am Institut für Soziologie der FSU Jena ist mein Hauptarbeitsplatz das UFZ in Leipzig. Das UFZ ist mit über 1.000 Mitarbeitenden eine weltweit einzigartige Einrichtung im Bereich der interdisziplinären Umweltforschung. Bereits bei seiner Gründung 1992 ging es vor dem Hintergrund der industriellen Umweltverschmutzung und der Bergbaufolgelandschaften in der Region darum, nachhaltige Wege im Umgang mit natürlichen Lebensgrundlagen aufzuzeigen. Das Department „Stadt- und Umweltsoziologie“ ist eines von fünf Einheiten des Themenbereichs „Umwelt und Gesellschaft“. Die Sozialwissenschaften stellen hier eine sichtbare Gruppe dar, aber unsere Arbeit 
findet immer in Kooperation mit den Naturund Ingenieurswissenschaften statt, denn das UFZ ist zu 95 Prozent ein naturwissenschaftliches Zentrum. Forschungsfragen werden in Kooperation mit Vertreterinnen anderer Disziplinen erarbeitet. Ohne diese Kooperation könnten wir nicht existieren. Da wir Teil der Helmholtz-Gemeinschaft sind, ist unsere Forschung eingebettet in die sogenannte Programmorientierte Forschungsförderung (POF) von insgesamt 19 Helmholtz-Zentren. Bei der POF handelt es sich um zentrenübergreifende Forschungsprogramme, die alle fünf, ab 2021 alle sieben Jahre neu geschrieben werden. Wir können uns also anders als an Universitäten unsere Themen nicht so frei aussuchen, da sie im Programm vorgegeben sind. Es bleiben aber noch genügend Spielräume.

SozMag: Können Sie etwas zu Ihren aktuellen Schwerpunkten sagen?

Groß: Unsere aktuellen Schwerpunkte erstrecken sich auf Lebensqualität in Städten im 21. Jahrhundert vor dem Hintergrund des Klimawandels und allgemein des globalen Wandels. Damit verbundene Themen richten sich auf Flächeninanspruchnahme und Ressourcennutzung für eine nachhaltige Gesellschaftsentwicklung. Soziologisch wichtig sind dabei die kulturellen Grundlagen und Paradoxien aktueller Transformationsprozesse wie der Energiewende. Neben vielen Facetten der umweltbezogenen
Stadtforschung hat unser Department eine lange Tradition in der Erforschung von Extremereignissen; ein Themenfeld, das gerade heute an Aktualität kaum zu übertreffen ist. Das heißt, wie wirken sich Überschwemmungen, Dürreperioden und Hitzewellen auf Gesellschaften aus und welche sozialen, technologischen und kulturellen Anpassungspotentiale gibt es hierfür? Wir überlegen aktuell, inwieweit die Corona-Krise konzeptuell hier hineinpasst und was für Lerneffekte im Vergleich $\mathrm{zu}$ anderen Krisensituationen, die wir im Umweltbereich intensiv untersucht haben, abgeleitet werden können. Eine wichtige Frage ist hier, inwieweit nachhaltige Gesellschaftsstrukturen, so wie sie bis jetzt gefördert wurden, Pandemien begünstigen. Die Debatten zu Ansteckungsrisiken in öffentlichen Verkehrsmitteln, die in urbanen Räumen bereits jetzt zu einer Zunahme in der individuellen PKW-Nutzung geführt haben, sind vielleicht erst der Anfang. Fragen nach individuellem Konsum, wie zum Beispiel von Einwegverpackungen im Zusammenhang mit neuen Hygiene-Vorstellungen, weisen auf bisher ungeahnte neue Spannungen zwischen verschiedenen Zielvorstellungen hin. Damit hängen dann soziologisch zentrale Themen der Governance, der Gerechtigkeit, von Alltagspraktiken oder klassische Fragen der Technikakzeptanz und der sozialen Konflikte zusammen. Momentan sind wir mit verschiedenen europäischen Projektpartner ${ }^{\star}$ innen dabei, eine Methodik zur 
Erforschung und Bestimmung gesellschaftlicher Rahmenbedingungen für Technologieimplementierungen am Beispiel der $\mathrm{CO} 2$-Speicherung zu entwickeln. Ein politisch heikles Feld, aber das macht es soziologisch natürlich umso interessanter.

SozMag: Mit welchen Themen haben Sie sich zuvor befasst?

Groß: Ich bin seit 2005 am UFZ und habe die ersten Jahre in der Altlastensanierung gearbeitet, dann kam ein Wechsel hin zur Energieforschung und seit den letzten Jahren wird das Thema Chemikalien in der Umwelt zunehmend wichtiger. Als roter Faden zieht sich durch meine Forschungen das Thema experimenteller Praktiken in Wissenschaft und Gesellschaft. Dazu gehören Umweltinnovationen und Technikentwicklungen im Kontext von gesellschaftlichen Prozessen sowie viele damit verbundene Unsicherheiten und paradoxe Entwicklungen. Die Corona-Krise hat uns vor Augen geführt, dass Entscheidungen unter Bedingungen des Nichtwissens eher den Normalfall darstellen. Umweltsoziologisch ist das aber ein alter Hut. Man muss lernen, mit unvermeidbaren Wissenslücken umzugehen, anstatt sie hinter irgendeiner politisch gewünschten Sicherheitsrhetorik zu verstecken.

SozMag: Inwiefern kann die Soziologie einen Umgang mit Wissenslücken vermitteln?

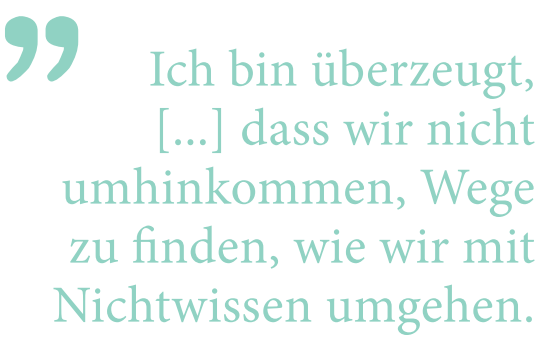

Groß: Die Soziologie kann zum einen Hinweise dafür liefern, dass Nichtwissen in Politik und Öffentlichkeit nicht per se als Dummheit oder Unverantwortlichkeit missverstanden werden. Ganz konkret geht es mir darum, die Wichtigkeit von Transparenz in der öffentlichen oder politischen Kommunikation zu vermitteln. Das heißt, zu erklären, dass bestimmte Dinge unter diesen oder jenen Bedingungen nicht gewusst werden können, oder zu vermitteln, dass beispielsweise Daten fehlen, um eine sinnvolle Risikoabschätzung zu machen. Dass diese Abschätzungen politisch gewollt dennoch vorgenommen werden, oft auf Basis weniger oder bloß geschätzter Daten, ist meines Erachtens unverantwortlich. Ich bin überzeugt - gerade aufgrund von Big Data und Co. -, dass wir nicht umhinkommen, Wege zu finden, wie wir mit Nichtwissen umgehen. Die Soziologie sehe ich dafür als wichtigen Wegbereiter.

SozMag: Die umweltsoziologische Forschung ist ein interdisziplinäres Unterfangen. Die Ansätze der Umweltsoziologie schließen an unterschiedliche Disziplinen wie die Geografie, Wirtschafts- und Naturwissenschaften an. 
Groß: Die Umweltsoziologie hat sich zumindest in Deutschland als interdisziplinäres Unterfangen entwickelt, was nicht unwesentlich damit zusammenhängt, dass umweltsoziologische Forschung für lange Zeit an außeruniversitären Forschungseinrichtungen vorangetrieben wurde. Das wiederum hängt damit zusammen, dass der Forschungsbereich an Universitäten in Deutschland bisher wenig institutionalisiert ist. Es gibt bis auf meine Stelle in Deutschland keinen Lehrstuhl, der in der Denomination das Wort „Umweltsoziologie" trägt. Es werden in den kommenden Jahren aber glücklicherweise noch einige Professuren mit der Denomination „Umweltsoziologie" dazukommen. Bisher ist die umweltsoziologische Forschung und Lehre an Universitäten in Deutschland allerdings - mit Ausnahme der Uni Hamburg und dem Klimazentrum um Anita Engels - Einzelpersonen zuzurechnen. Durch die methodischen, aber vor allem theoretischen Versuche der Umweltsoziologie, der außersozialen Umwelt Gehör zu verschaffen, hat die Disziplin immerhin keine große Scheu gehabt, zusammen mit den Natur- und Ingenieurswissenschaften komplexe Umweltprobleme zu bearbeiten. Was die verschiedenen Standorte der umweltsoziologischen Forschung aktuell stark verbindet, sind die Themen Klimawandel und, insbesondere in Deutschland, die Energiewende.
SozMag: Welche Herausforderungen ergeben sich für die Umweltsoziologie aus der Interdisziplinarität? Welche Chancen birgt sie?

Groß: Unumgänglich sorgt die Zusammenarbeit unterschiedlicher Disziplinen für Missverständnisse. Ein häufiges Phänomen ist, dass die Soziologie im Besonderen und die Gesellschaftswissenschaften im Allgemeinen von naturwissenschaftlicher Seite gerne als Mittel zur gesellschaftlichen Akzeptanzbeschaffung missverstanden werden. Da steckt in der Regel keine böse Absicht dahinter, weshalb ich auch gerne immer wieder gegen diese Windmühle anrenne. Vieles amüsiert mich regelrecht. Etwa wenn Ingenieur*innen mit bestem Wissen und Gewissen eine Technologie entwickeln, von der sie sich zum Beispiel große Effizienzsteigerungen erhoffen, die für eine nachhaltige Gesellschaft durchaus wichtig sein könnten. Dann kommen sie zu uns und fragen, wie man das nun gesellschaftlich implementieren könnte, wie man psychologische, politische oder kulturelle Widerstände abflachen oder gar ausmerzen könnte. Ich drücke das hier etwas überspitzt aus, ganz so naiv sind die Naturwissenschaften sicher nicht und in vielen transdisziplinären Forschungsverbünden geht man Probleme erfolgreich gemeinsam an. Aber Missverständnisse über das, was die Soziologie kann und soll, die gibt es immer wieder. 


\section{Die Auseinandersetzungen mit den Naturwissenschaften führen auf Seiten der Soziologie zu neuen Einsichten und ermöglichen, die eigene Disziplin, ihre Potentiale und
Grenzen besser verstehen zu lernen.}

Gleichzeitig sehe ich die Chancen, die in der Interdisziplinarität liegen: Die Auseinandersetzungen mit den Naturwissenschaften führen auf Seiten der Soziologie $\mathrm{zu}$ neuen Einsichten und ermöglichen, die eigene Disziplin, ihre Potentiale und Grenzen besser verstehen zu lernen. Es ist schade, dass diese Perspektivwechsel in der universitären Lehre nicht besser verankert werden. Es ist im Curriculum der Soziologie kaum denkbar, dass Studierende zum Beispiel Seminare in Geologie belegen. Allein die, wie auch immer, vage Kenntnis der Methoden anderer Disziplinen kann schon vor soziologischen Schnellschüssen und Fehlzuschreibungen schützen.

Wer in interdisziplinären Kontexten wie der Umweltsoziologie arbeitet, lernt darüber hinaus schnell, sich nicht an eine soziologische Theorie oder einen methodischen Zugang zu klammern. Man geht die Sache vom Problem aus an. Man muss sehen, wie man zur Lösung relevante Daten erarbeiten kann und wie diese sinnvoll konzeptuell gerahmt werden können. Das heißt, man muss sich manchmal in Methoden oder zumindest in Versatzstücke aus Theorien einarbeiten, auch wenn sie einem nicht immer liegen mögen. Auch hier finde ich schade, dass heute in der universitären Ausbildung in unserem Fach oft noch theoretischer Einseitigkeit gefrönt wird - und die Methodenkluft zwischen sogenannten "Qualis" und "Quantis" scheint mir auch nicht kleiner zu werden.

Die Chancen der Interdisziplinarität habe ich bereits in meiner frühen Tätigkeit gleich nach meiner Promotion in einem Nachwuchsgruppenprojekt zu „Realexperimenten" zusammen mit einem Naturwissenschaftler an der Uni Bielefeld erfahren. Am UFZ war ich anfangs in der Altlastensanierung tätig - das heißt, es ging um große Sanierungsprogramme des industriell verunreinigten Bodens. Zur Entwicklung von standortspezifischen Nachhaltigkeitsindikatoren haben wir beispielsweise Befragungen durchgeführt, um herauszufinden, welche konkreten Probleme es in der Region und den zu sanierenden Industriestandorten gibt. Darauf aufbauend haben wir dann zusammen mit Computermodellierer*innen ein Entscheidungsunterstützungstool für den Bereich Altlastensanierung entwickelt. Erst auf den zweiten Blick hatte das etwas mit dem zu tun, was man aus der universitären Forschung und Lehre kennt; wir haben 
vielmehr mit Versatzstücken aus der Soziologie, insbesondere mit verschiedenen Befragungsmethoden gearbeitet. Aber gerade darüber bin ich schließlich zur Soziologie des Nichtwissens gekommen. Man wusste nicht, welche Stoffe im Boden sind, und dennoch mussten die Verantwortlichen ständig Entscheidungen treffen - bei denen sie wussten, dass sie vieles nicht wussten. Diese Form der Zusammenarbeit mit Fachfremden hat letztendlich meine eigene Forschung immer beflügelt.

SozMag: Mit Greta Thunbergs Schulstreik und globalen Bewegungen wie Fridays for Future oder Extinction Rebellion sind die Themen Klima- und Umweltschutz und die Fragen danach, in welchem Verhältnis wir zur Natur leben (wollen), ins gesamtgesellschaftliche Bewusstsein gerückt. Gleichzeitig ist der Ton rauer geworden. Der Druck auf Politiker*innen, die Umwelt zu schützen, wächst, sogenannte Klimaleugner ${ }^{*}$ innen nutzen die Themen für ihre eigene politische Agenda. Inwiefern kann die Umweltsoziologie zu diesen Diskursen beitragen?

Groß: Die Umweltsoziologie ist in gewisser Weise ohne die Umweltdiskurse seit den 1970er Jahren nicht denkbar. Von daher vermutet man natürlich eine enge Verbundenheit mit der Umweltbewegung - und personell gesehen ist und war das sicherlich auch oft so. Eine Parteiergreifung wird allerdings meines Erachtens immer schwieriger. Im Namen der Natur werden Windräder gebaut, im Namen der Natur wird aber auch dagegen protestiert. In meinen Augen ist es falsch, sich schon im Vorhinein auf eine Perspektive zu einigen oder sich auf eine Seite zu schlagen. Kritische Soziologie bedeutet für mich nicht, sich Zielen sozialer Akteur*innen anzuschließen, sondern eine distanzierte Perspektive einzunehmen und radikal gegen den Strich zu denken.

Ich glaube, dass es der Umweltsoziologie in erster Linie darum gehen sollte, in diesem Sinne kritische und empirisch fundierte Analysen zu betreiben. Wenn Fridays for Future-Advokat*innen sagen, „science is real" und "Hört auf die Wissenschaft" Aussagen, die auf einen plötzlichen Wandel zu einer wie auch immer gearteten Wissenschaftsbegeisterung hindeuten -, dann sollte uns das aufhorchen lassen. Soziologisch wird oft übersehen, dass

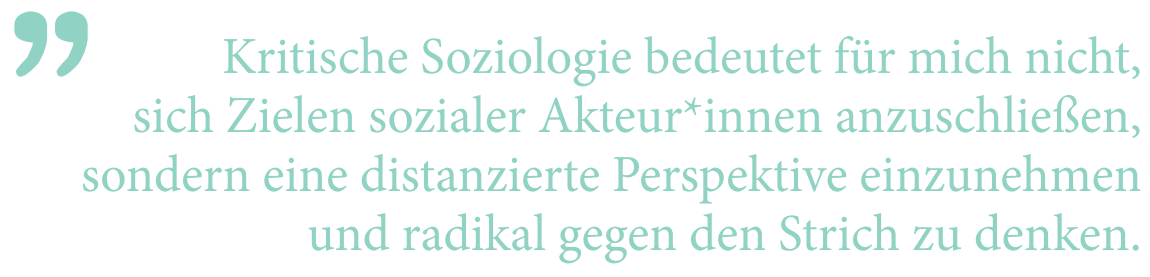


Fridays for Future bestenfalls jene Stränge der Wissenschaften im Blick hat, die medial laut hörbar sagen, was die Positionen von Greta Thunberg und ihren Anhänger ${ }^{*}$ innen stützen. Es kann also nicht Aufgabe der Soziologie sein, Argumentverstärker für Fridays for Future zu werden, und die, die sich der Bewegung nicht anschließen wollen, als Leugner*innen oder Skeptiker*innen zu brandmarken. Mit solchen aus dem öffentlichen Diskurs aufgegriffenen Kampfbegriffen untergräbt die Soziologie zum einen ihre eigene wissenschaftliche Position. Zum anderen sollte es darum gehen, diese Begriffe innerhalb des Diskurses zu verorten und die ihnen zugrundeliegenden Prozesse und Entwicklungen aufzuzeigen. Schaut man sich die Begriffe einmal genauer an, dann klingt der Erste der „Leugner*innen“ nach religiös Abgefallenen, die das Evangelium verneinen oder nicht akzeptieren wollen. Also die Ungläubigen oder Ketzer*innen, die der wahren Religion nicht folgen. Da sollte man in soziologischen Beschreibungen vorsichtig mit sein. Aber auch die Rede von den Skeptikerinnen ist soziologisch bedenklich - nicht zuletzt, da Robert Merton vor dem Hintergrund der politisch gesteuerten Nazi-Wissenschaft sowie der sowjetischen Agrarwissenschaft seit den 1930er Jahren innerhalb der von ihm formulierten Normen die freie Wissenschaft als „organisierten Skeptizismus“ charakterisiert hat.
Eine kritische Soziologie könnte den öffentlichen Diskurs befruchten, indem sie aufzeigt, was für quasi-religiöse und oft auch anti-demokratische Argumentationsmuster Fridays for Future und andere Gruppierungen vorlegen. Ich habe allerdings den Eindruck, das traut man sich bisher nicht so recht.

SozMag: Über die Umwelt zu sprechen, ist politischer als je zuvor. Verstehen Sie die Umweltsoziologie auch als eine politische Wissenschaft?

Groß: Die Umweltsoziologie ist gelegentlich auch eine politische Wissenschaft, was ich aber zunehmend kritisch betrachte. Ich glaube, vor zehn Jahren hätte ich Ihre Frage noch anders beantwortet. Mittlerweile sehe ich große Probleme, dass sich nicht nur die Umweltsoziologie, sondern auch viele andere Stränge unserer Disziplin viel $\mathrm{zu}$ oft vor politische Karren spannen und sich instrumentalisieren lassen - und nicht immer scheint man das zu merken. Zumindest sehe ich darin eine Gefahr, denn allzu großes Überzeugungstäter*innentum schadet der Wissenschaftlichkeit auf Dauer. Ich finde, dass sich insbesondere die Umweltthematik mit all ihren naturwissenschaftlichen und gesellschaftlichen Unsicherheiten, paradoxen Effekten und dem oft schnellen Wandel des Wissens über Naturzustände soziologisch gesehen am wenigsten eignet, moralisch, politisch oder ethisch eindeutig Stellung zu beziehen. Als 
Privatperson kann ich das tun, aber nicht als empirisch und analytisch arbeitende ${ }^{\star} r$ Soziolog ${ }^{\star}$ in. Das trenne ich mittlerweile, so gut es geht, sehr streng, was sicherlich auch mit meiner alltäglichen Zusammenarbeit mit den Naturwissenschaften zu tun hat.

SozMag: Warum plädieren Sie für eine solche Trennung? Welche Gefahr(en) sehen Sie für politische bzw. sich politisierende Wissenschaften?

Groß: Diese Trennung und kritische Distanz zu tagespolitischen Umweltdiskursen verbaut einem nicht die Sicht auf überraschende und kontraintuitive Forschungsergebnisse. Mal eben auf die Wissenschaft hören, wie es unsere Fridays for Future-Protagonist ${ }^{\star}$ innen fordern, das ist - zumindest soziologisch gesehen - Kokolores. Wenn man sich auf der moralisch überlegenen Seite wähnt und schon im Vorhinein weiß, wer (naturwissenschaftlich) Recht hat, wer die Guten und wer die Bösen sind, wie genau eine gute, nachhaltige oder richtige Gesellschaftsentwicklung aussehen muss, dann ist es auch absehbar, was die soziologischen Ergebnisse sind. Im Grunde braucht es so keine Forschung, wenn immer schon vorher feststeht, dass irgendein Schreckgespenst an einer Misere schuld ist oder dass nur dieses oder jenes Verhalten besonders ökologisch, gut oder nachhaltig ist. Die Umweltsoziologie sollte die Analyse sozial-ökologischer Prozesse, also die gesellschaftliche Entwicklung in ökologischen, technischen und sozialen Kontexten, mit sauber erarbeiteten empirischen Daten vorantreiben und nicht vorab politische Aktivitäten fördern oder verhindern. Wir haben genug damit zu tun, die mit ökologischen Problemen verbundenen Bedingungen menschlichen Fühlens, Denkens und Handelns sowie deren Folgen und oft nicht intendierte Nebenfolgen aufzuzeigen und zu verstehen.

\section{LITERATUR}

Beck, Ulrich (1986): Risikogesellschaft - Auf dem Weg in eine andere Moderne. Frankfurt am Main: Suhrkamp.

Brand, Karl-Werner (2014): Umweltsoziologie. Entwicklungslinien, Basiskonzepte und Erklärungsmodelle. Weinheim: Beltz-Juventa.

Catton, William R./Dunlap, Riley E. (1978): Environmental Sociology: A New Paradigm. In: The American Sociologist Jg. 13/1, S. 41-49.

Linde, Hans (1972): Sachdominanz in Sozialstrukturen. Tübingen: Mohr Siebeck.

Luhmann, Niklas (1986): Ökologische Kommunikation. Opladen: Westdeutscher Verlag.

Merton, Robert K. (1973 [1942]): The Normative Structure of Science. In: Merton, Robert K.: The Sociology of Science: Theoretical and Empirical Investigations. Chicago: University of Chicago Press, S. 267-280. 


\section{WEITERFÜHRENDE LITERATUR}

Becker, Egon/Jahn, Thomas (Hrsg.) (2006): Soziale Ökologie: Grundzüge einer Wissenschaft von den gesellschaftlichen Naturverhältnissen. Frankfurt am Main: Campus.

Bleicher, Alena/Groß, Matthias (2010): Sustainability Assessment and the Revitalization of Contaminated Sites: Operationalizing Sustainable Development for Local Problems. In: The International Journal of Sustainable Development \& World Ecology Jg. 17/1, S. 57-66.

Block, Katharina (2016): Von der Umwelt zur Welt: Der Weltbegriff in der Umweltsoziologie. Bielefeld: transcript.

Davidson, Debra J./Groß, Matthias (Hrsg.) (2018): The Oxford Handbook of Energy and Society. Oxford: Oxford University Press.

Göbl, Magdalena (2019): Die Paradoxie der Ökologie. Baden-Baden: Nomos.

Groß, Matthias (2010): Ignorance and Surprise: Science, Society, and Ecological Design. Cambridge/MA: MIT Press.

Groß, Matthias (Hrsg.) (2011): Handbuch Umweltsoziologie. Wiesbaden: Springer VS.

Groß, Matthias (2014): Experimentelles Nichtwissen: Umweltinnovationen und die Grenzen sozial-ökologischer Resilienz. Bielefeld: transcript.

Groß, Matthias (2018): Energetische Soziologie. In: Holstenkamp, Lars/Radtke, Jörg (Hrsg.): Handbuch Energiewende und Partizipation. Wiesbaden: Springer VS, S. 177-194.

Kropp, Cordula/Sonnberger, Marco (2021, im Erscheinen): Einführung in die Umweltsoziologie. Baden-Baden: Nomos.

Das Interview führte Cathrin Mund. Es wurde von Tanja Strukelj lektoriert.

\section{In Zeiten von Dauerkrise und Untergangslust: Wie ist eine wünschens- werte Zukunft überhaupt noch vorstellbar?}

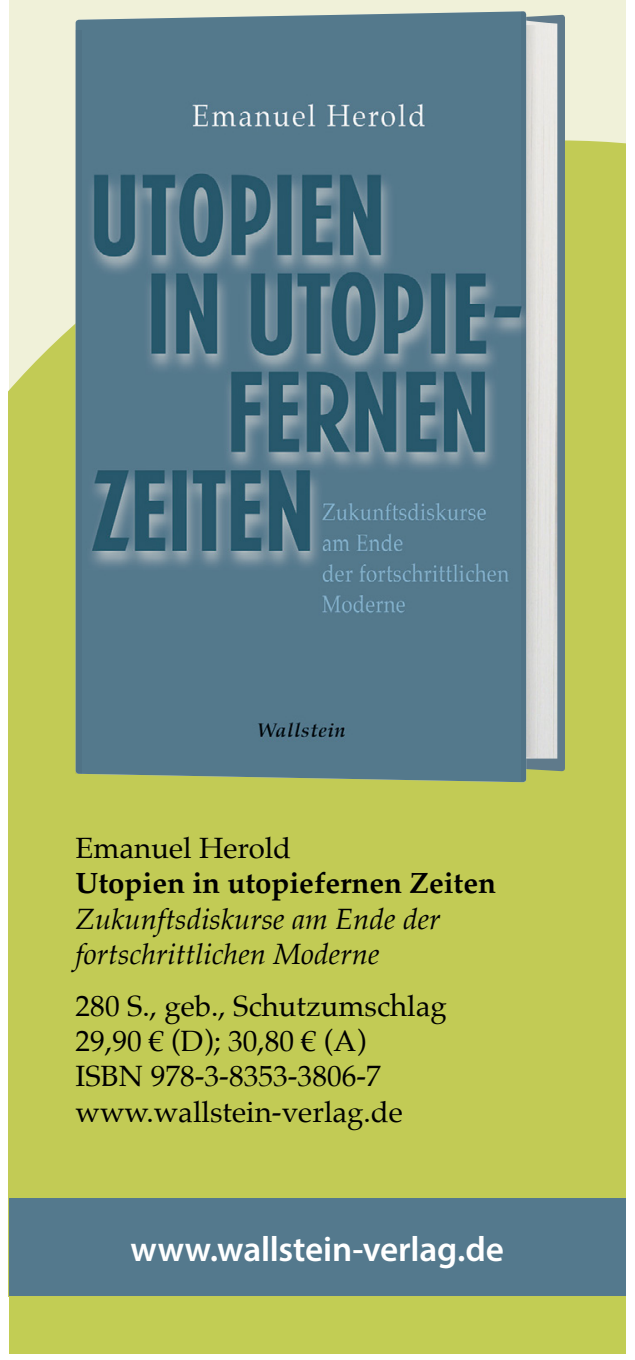




\section{„Es braucht einen tiefgreifenden sozial-ökologischen gesellschaftlichen Wandel“}

\section{Ein Expertininterview mit Anna-Katharina Brenner}

von Andreas Schulz

SozMag: Du bist studierte Sozialwissenschaftlerin und hast nach deinem Soziologiestudium im Master Soziale Ökologie studiert. Wie bist du zu dem Themenfeld gekommen, was interessiert dich an dem Themenkomplex Umwelt und Gesellschaft besonders und kannst du dein explizites Forschungsinteresse skizzieren?

Brenner: Ich bin begeisterte Soziologin, dennoch haben mir während meines Studiums immer wieder Konzepte gefehlt, die für mich die Bedingungen gesellschaftlichen Lebens greifbarer machen. Fündig bin ich in der Sozialen Ökologie geworden, in der die gegenseitige Bedingtheit von Gesellschaft und Umwelt im Zentrum aller Fragen steht. Grundlegende Konzepte der Wiener Schule der Sozialen Ökologie sind dabei die Kolonisierung natürlicher Systeme, also deren gesellschaftliche Aneignung, um sie nutzbar machen zu können, sowie das Konzept des gesellschaftlichen Stoffwechsels bei Haberl und Kolleg*innen (2016). Der gesellschaftliche Stoffwechsel beschreibt die kontinuierliche Versorgung der Gesellschaft mit Energie und biophysischen Materialien, die das gesellschaftliche Leben, so wie wir es kennen, überhaupt erst möglich machen. Dabei entstehen auch Abfallstoffe und Emissionen, die wieder an die Umwelt und den Menschen abgegeben werden, wie zum Beispiel Luftverschmutzung oder CO2-Emissionen. Die Intensität des gesellschaftlichen Stoffwechsels ist abhängig von dem dominierenden Gesellschaftssystem. Im gegenwärtigen globalen Kapitalismus hat die Ressourcennutzung 


\section{[W]ir brauchen einen radikalen Wandel in der Art, wie wir Ressourcen nutzen, aber auch in der Art, wie wir sie verteilen.}

solche Ausmaße angenommen, dass sie die planetaren Grenzen übersteigt und die Klimakrise vorantreibt (vgl. Brand/ Wissen 2017). Dabei sind die natürlichen Ressourcen sowie ihre Vor- und Nachteile nicht gleich in den Gesellschaften verteilt. Es hat sich gezeigt, dass neben Bevölkerungs- und Wirtschaftswachstum vor allem wohlhabende Haushalte weltweit den größten negativen Einfluss auf die Umwelt haben (vgl. Wiedmann et al. 2020). Das heißt, wir brauchen einen radikalen Wandel in der Art, wie wir Ressourcen nutzen, aber auch in der Art, wie wir sie verteilen. Die Kernfrage dabei ist: Wie können wir unseren Ressourcenverbrauch einschränken und somit die Folgen der Klimakrise zumindest abschwächen, ohne bestehende Ungleichheiten zu vertiefen oder neue Ungleichheiten zu produzieren (vgl. O’Neill et al. 2018)?

SozMag: Welchen Stellenwert hat Interdisziplinarität in der Sozialen Ökologie?

Brenner: Einen riesengroßen. Die Soziale Ökologie ist ohne interdisziplinäres Arbeiten und Denken überhaupt nicht möglich. Beispielsweise basieren die Grundzüge der Wiener Schule der Sozialen Ökologie auf systemtheoretischen Ansätzen (Luhmann), die später um betriebswirtschaftliche und naturwissenschaftliche Ansätze, wie die Material- und Energieflussrechnungen, erweitert worden sind. Das heißt, dass die Soziale Ökologie interdisziplinär ausgerichtet ist, um reale, problemzentrierte Fragen rund um das Verhältnis von Gesellschaft und Natur zu beantworten. Interdisziplinäre Kommunikation spielt dabei eine sehr große Rolle. Um so zu arbeiten, ist es absolut notwendig, deine eigene disziplinäre Vergangenheit zu reflektieren und dir immer wieder ins Bewusstsein zu rufen, dass Begrifflichkeiten, die ich verwende, in anderen Disziplinen andere Bedeutungen haben können. Das heißt, interdisziplinäres Arbeiten ist ein ständiger Aushandlungsprozess zwischen verschiedenen Perspektiven: Wie meine ich das? Wie verstehst du das? Auf was können wir uns einigen, um eine spezifische Fragestellung zu bearbeiten? In der Realität haben die meisten Forscher*innen im interdisziplinären Arbeiten allerdings trotzdem entweder einen sozialwissenschaftlichen oder einen naturwissenschaftlichen Schwerpunkt. Bei uns bedeutet das, dass sich die eine Gruppe eher der Industrial Ecology angehörig fühlt, die sich vor allem mit der Quantifizierung bestehender Material- und Energieflüssen und Beständen auseinandersetzt. Diese Herangehensweise 


\section{Anna-Katharina Brenner}

Anna-Katharina Brenner ist Dissertantin der Doktoratsschule „Transition to Sustainability", die auf inter- und transdisziplinäre Forschung im Feld der nachhaltigen Entwicklung spezialisiert ist (Universität für Bodenkultur in Wien). Sie hat 2019 ihren Master in Human- und Sozialökologie in Wien abgeschlossen. In ihrer Masterarbeit hat sie sich mit Umweltgerechtigkeit in Österreich beschäftigt. Nach einem kurzen Abstecher ans Institut für Soziologie der Universität Wien, bei dem sie ihren Fokus

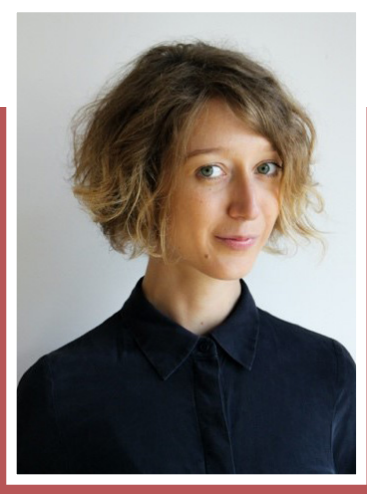
auf kritische Stadtforschung entwickelte, ist sie nun zurück am Institut für Soziale Ökologie als wissenschaftliche Mitarbeiterin. Ihre Forschungstätigkeiten umfassen folgende Gebiete: Gesellschaftliche Naturverhältnisse, Sozialer Metabolismus, Umweltgerechtigkeit, Urbane Politische Ökologie und Kritische Stadtforschung.

gibt Einblicke in die Intensität des gesellschaftlichen Einflusses auf die natürliche Umwelt und hat den Vorteil, dass die Zahlen einfacher politisch kommunizierbar sind als beispielsweise ideologisch-normative Forderungen. Die andere Gruppe fühlt sich eher der Politischen Ökologie zugehörig, die wiederum die Entstehung und Verteilung von Ressourcennutzung in den Blick nimmt. Ich selbst möchte mich weder ausschließlich der einen noch der anderen Disziplin zuordnen. Ich habe auch in meiner Masterarbeit zu Umweltgerechtigkeit sozialmetabolisch quantitativ gearbeitet und bin dabei doch der grundlegend sozialwissenschaftlichen Frage nach der in der Gesellschaft ungleichen Verteilung von Luftverschmutzung nachgegangen. Ich sehe mich da eher in der Mitte, ich finde Zahlen als politisches Kommunikationsmittel wichtig, dennoch steht für mich die Frage im Zentrum: Durch welche gesellschaftlichen Prozesse entstehen aktuelle dominante sozial-ökologische Prozesse und werden dabei durch Pfadabhängigkeiten immer wieder reproduziert. Und welche Möglichkeiten gibt es, diese Prozesse zu transformieren und dabei neben einer low-carbon future auch die Richtung einer sozial-ökologisch gerechteren Gesellschaft einzuschlagen.

SozMag: Auf welchen konzeptionellen Hintergründen basiert deine derzeitige Forschung und Arbeit am Institut für Soziale Ökologie an der Universität für Bodenkultur Wien (BOKU)? 
Brenner: Ich bin angestellt in dem ERC-Projekt "Understanding the Role of Material Stock Patterns for the Transformation to a Sustainable Society (MAT STOCKS)“. Im Zentrum des Projekts steht der, Stock-Flow-Service Nexus' (Haberl et al. 2017). Die Idee dabei ist, dass Menschen auf sozial-ökologische Services angewiesen sind, um am Alltagsleben teilhaben zu können. Diese Services sind zum Beispiel, ein Dach über den Kopf zu haben, wenn es dunkel ist, das Licht anzumachen, eine angenehme Raumtemperatur zu haben, mobil sein zu können, aber auch Zugang zu Naherholung und frische, schadstofffreie Luft zu atmen usw. Um diese sozial-ökologischen Services wahrnehmen zu können, braucht es die biophysische Bereitstellung von Beständen (stocks) wie zum Beispiel Häuser, Infrastrukturen; also eine Form der gebauten Umwelt, die in ihrem Zusammenspiel die räumlich spezifische biophysische Struktur von Gesellschaften bilden. Es braucht aber auch Materialund Energieflüsse, zum einen, um diese Bestände überhaupt erst aufzubauen, zum anderen, um sie dann zu nutzen. Wir können also erst das Licht in unserer Wohnung anschalten, wenn das Haus und die nötigen Leitungen sowie eine Glühbirne und ein Lichtschalter gebaut bzw. installiert worden sind (stocks) und dann Strom durch diese Leitungen fließt (flows) (vgl. Kalt et al. 2019). Ein großer Teil des Projekts widmet sich den Berechnungen globaler Materialbestände (stocks). In meinem
Forschungsschwerpunkt beschäftige ich mich mit Städten und ihrem Umland. Meine Aufgabe innerhalb des Projektes ist es, für den städtischen Raum für Wien und Umland mögliche Transformationspfade für bestehende, nicht-nachhaltige StockFlow-Service Nexus in Richtung nachhaltigere zu identifizieren und auf ihre Anwendbarkeit zu untersuchen.

So hat Wien sich historisch durch viele kluge Maßnahmen zu einem Paradebeispiel für städtische Nachhaltigkeit mit einer hohen Lebensqualität entwickelt. Dennoch können wir sehen, dass die Stadt durch tief verwurzelte Pfadabhängigkeiten daran gehindert wird, progressivere Maßnahmen zum Schutz des Klimas- und diesbezüglich auch zum Schutz der Menschen umzusetzen. Ein Beispiel dafür ist der Autoverkehr. In den letzten Jahrzehnten hat die Stadt Wien viele Maßnahmen umgesetzt, um vor allem den öffentlichen Verkehr, aber auch das Zufußgehen oder Fahrradfahren attraktiver zu machen. Gleichzeitig sind Maßnahmen umgesetzt worden, wie zum Beispiel Parkraumbewirtschaftung, die das Autofahren unattraktiver gemacht haben. Das hat dazu geführt, dass der Umweltverbund, das sind öffentlicher Verkehr, Fußgänger*innen und Fahrradfahrer* innen, stärker geworden ist. Dennoch werden immer noch 56 Prozent aller zurückgelegten Personenkilometer in Wien mit dem Auto zurückgelegt (vgl. Gassner et al. 2020), was auf eine langanhaltende Autoabhängigkeit des Mobilitätsystem in 
Wien hinweist. Vor allem meine Kollegin Doris Virág leistet da im Bezug zur Berechnung des Stock-Flow-Service Nexus großartige Arbeit, indem sie den Materialbedarf von Mobilitätsinfrastrukturen und deren Instandhaltung mit Energieverbrauch und Emissionsintensität diverser Mobilitätsformen in Wien verknüpft. Nicht wenig überraschend benötigt Automobilität die meisten Infrastrukturen und somit Materialflüsse für Instandhaltung sowie Energieflüsse für die Fortbewegung. Und dann komme ich ins Spiel. Wenn wir es jetzt schwarz auf weiß haben, dass Mobilität mit dem Auto nicht nachhaltig ist, lassen sich Fragen stellen, etwa welche Stadtplanungskonzepte es gibt, die diese Autoabhängigkeit aufbrechen können und welche gesellschaftlichen Strukturen mögliche Transformationsversuche zum Teil aktiv verhindern - weil sie eben vor allem in Pfadabhängigkeiten verhaftet bleiben. Dabei hat sich gezeigt, dass die gebaute Umwelt ein zentrales Element darstellt. Wie Raum gestaltet ist, hat einen maßgeblichen Einfluss auf unsere Mobilitätsmuster; vice versa beeinflussen dominante Mobilitätsmuster die räumliche Struktur von Städten und Umland.

Die gebaute Umwelt ist aber auch für andere sozial-ökologische Services zentral: Sie bedingt zum Beispiel die Bereitstellung von Grünräumen in Städten, die wiederum mit einer Vielzahl an weiteren Services einhergeht, wie etwa frischer Luft, Kühlungseffekten und einer Stabilisierung des
Mikroklimas. Grünräume dienen zudem auch als Naherholungsorte, stärken gesellschaftlichen Zusammenhalt, sind Orte der sozialen Interaktion oder der Entspannung, kurz gesagt, sie tragen zur Verbesserung der urbanen Lebensqualität bei. Auch historisch gesehen ist die Bereitstellung von urbanen Grünflächen oft dazu benutzt worden, um soziale Missstände räumlich auszugleichen (vgl. Loughran 2020). Früher wurden Grünflächen erschaffen, um die miserable Wohnverhältnisse auszugleichen, vor allem während der Industrialisierung, heute wird die Erschaffung neuer (Grün) räume und Umgestaltung bestehender (Grün)räume als eine Lösung der Klimakrise gesehen. Dennoch hat sich gezeigt, dass gerade die Bereitstellung von Grünflächen in Städten mit der Entstehung neuer Ungleichheiten einhergehen kann. Stichwort: ,Green Gentrification' (Gould/Lewis 2017). Das heißt, dass durch die Erschaffung von Grünräumen die umliegenden Mietpreise so steigen, dass der direkte Zugang zu den sozial-ökologischen Services durch die Grünräume einer kleinen wohlhabenden Gruppe vorbehalten ist. Kurzum, in meiner Dissertation treibt mich vor allem die Frage nach der gesellschaftlichen Produktion von (städtischem) Raum inklusive seiner sozial-ökologischen Prozesse um. Vor allem die kritische Stadtforschung bietet für meine Fragestellung fruchtbare Anschlusspunkte. Wichtig ist für mich der Historische Institutionalismus, vor allem in Bezug auf Stadtplanungsgeschichte (vgl. 
Sorensen 2015), genauso wie Erkenntnisse zu Transformationspotentialen in Alltagspolitiken (z.B. Zografos et al. 2020). Dabei beziehe ich mich unter anderem auch auf neomarxistische Arbeiten wie die von David Harvey (2009)mit seinen Konzepten vom ,spatial fix - die Erschaffung neuer Räume, um Krisen des Kapitalismus zu überwinden. Die Ergebnisse zu den dadurch entstehenden "geographisch ungleichen Entwicklungen" bieten mir interessante Einblicke in die zugrundeliegenden polit-ökonomischen Strukturen der sozialen Produktion von Raum.

Doch um zu verstehen, wie diese ungleiche Verteilung von Vor- und Nachteilen im städtischen Raum und darüber hinaus, durch bestimmte Interessens- und Machtstrukturen erschaffen und auch erhalten wird, sind für mich auch Arbeiten der Urbanen Politischen Ökologie (vgl. Heynen et al. 2006; Swyngedouw/Heynen 2003; Swyngedouw 2006) sowie die wissenschaftlichen Aufarbeitung der EnvironmentalJustice-Bewegung (z.B. de Schutter et al. 2017; Martinez-Alier et al. 2014) bereichernd.

SozMag: Wie sieht eine Forschungsfrage bzw. Projekt in deinem Bereich aus?

Brenner: Ganz konkret beschäftige ich mich gerade mit der Frage, wie der Service der Mobilität in einer Stadt wie Wien ressourcenschonender gestaltet werden könnte, ohne Menschen die Möglichkeit zu nehmen, am Alltagsleben teilhaben zu können. So ist es meines Erachtens keine gute Idee, das Autofahren durch restriktive Maßnahmen wie die höhere Besteuerung von Benzin einzuschränken zu wollen, ohne durch den Ausbau des öffentlichen Verkehrs ein Ausgleichsangebot zu erschaffen. Daraus können neue Ungleichheiten wie zum Beispiel ,fuel poverty' entstehen. Um zur Arbeit zu kommen, um Lebensmittel einzukaufen und Freizeitaktivitäten nachgehen zu können, Freund ${ }^{\star}$ innen zu treffen, Kindergärten und Schulen zu besuchen, also öffentliche Einrichtungen zu nutzen, bedarf es der Möglichkeit, von einem Ort zum anderen zu kommen. Das ist in unserem Sinne des Stock-Flow-Service Nexus der Mobilitätsservice. Stadtplanung spielt dabei eine große Rolle, denn je dichter die Mobilitätsziele räumlich verteilt sind, desto geringer ist das Mobilitätsaufkommen. Es gibt da unterschiedliche Instrumente. Aktuell viel diskutiert werden die Superblocks, ein Stadtplanungsmodell, das bereits in Barcelona angewendet wird. Superblocks sind $400^{\star} 400 \mathrm{~m}$ große planerische Einheiten, vergleichbar mit Stadtvierteln, Kieze oder auf Wienerisch ,Grätzeln. Innerhalb dieser Einheit wird die Mobilität mit dem Auto durch Umbauten und verkehrsplanerische Maßnahmen eingeschränkt und aktive Mobilitätsformen wie Zufußgehen und Fahrradfahren gefördert. An den Außenkanten der Superblocks zirkulieren ein gut ausgebautes öffentliches Verkehrsnetz und Autos. Innerhalb der Superblocks 
werden Infrastrukturen, die für die Autos erschaffen worden sind, zurückgebaut und die neu gewonnen Flächen im besten Fall attraktiv für die öffentliche Nutzung gestaltet. Die Gestaltung dieser Räume kann im Idealfall, unter der Einbeziehung der Anwohner*innen, demokratisch organisiert werden. Begrünung spielt da vor allem in dicht bebauten städtischen Räumen eine große Rolle. Im Idealfall stehen wichtige öffentliche Leistungen, wie medizinische Versorgung, Kindergärten, Schulen usw., aber auch neu erschaffene Naherholungsorte, wie eine Bank unter einem Baum sowie Einkaufsmöglichkeiten der Grundversorgung, in kurzer Distanz zu Verfügung (siehe zu dem Superblockkonzept Rueda 2019). Es ist ein interessantes Konzept, das die Gestaltung von städtischem Raum grundlegend verändern und dabei vielleicht autoabhängige Strukturen („,ardependency" siehe dazu zum Beispiel: Mattioli et al. 2020; Newman et al. 2016) aufbrechen könnte. In unserer Forschung bauen wir dabei auf eine erste explorative Erhebung auf, die die Anwendbarkeit von Superblocks auf einer stadtplanerischen und verkehrsplanerischen Ebene untersucht hat (projekte.ffg.at/projekt/3039713). Uns beschäftigt dabei einerseits die quantifizierbaren sozial-ökologischen Vorteile, die durch die Umsetzung von Superblocks in bestimmten Gegenden Wiens zu erwarten wären, andererseits die Frage, wie realistisch die Umgestaltung von Raum ist. Dabei fokussieren wir uns bei der Analyse auf vergangene und aktuelle Stadtentwicklungsziele, aber vor allem auch auf Alltagspolitiken. Insbesondere bestehende Pfadabhängigkeiten, die das bestehende carbon-intensive System unterstützen und progressivere Maßnahmen zum Schutz des Klimas verhindern, sehe ich hier als ein großes Hindernis. Gregory C. Unruh (2000) hat diesbezüglich den Begriff des „Carbon Lock-in“ geprägt. Es braucht ein ,unlock' von beispielsweise bestehenden lokalen Politiken, um die Barrieren des Carbon-lock-in zu überwinden. Dabei sehen wir in Alltagspolitiken Transformationspotential. Alltagspolitiken können einerseits starrhalsig jegliche Veränderungen ablehnen, andererseits sind sie aber auch umkämpfte Gebiete, in denen verhärtete Strukturen ausgeweitet werden können. Dadurch ergeben sich vielleicht Handlungsspielräume, um progressivere Stadtplanungsinterventionen wie die Superblocks in einer Stadt wie Wien umzusetzen.

SozMag: Wie wird es dann in dem Projekt und in deinem PhD weitergehen? Welche Relevanz hat dein Forschungsfeld im gegenwärtigen sozialpolitischen Umweltdiskurs?

Brenner: Das ist und bleibt ein spannender Aushandlungsprozess zwischen den verschiedenen Schwerpunkten der sozial-ökologischen interdisziplinären Forschung, in der sich in den kommenden Jahren noch viel tun wird. In meinen 


\section{9 Ich glaube, wir werden uns von dem Gedanken verabschieden müssen, dass technologische Innovationen die Lösung des Problems sein können.}

kommenden Papers wird es um verschiedene Transformationstiefen gehen. Superblocks sind in ihrem Einfluss ja auf ihren Standort begrenzt, das heißt, dass ihre Eingriffstiefe beschränkt ist. Neue Stadtentwicklungsprojekte und aktuelle Mobilitätsinfrastrukturmaßnahmen bieten sich an, um tiefere Transformationsebenen zu untersuchen. Meiner Meinung nach wird interdisziplinäre Forschung im Bereich Umwelt und Gesellschaft noch lange Zeit sehr relevant bleiben, da tiefergreifendere Versuche, die sozial und ökologische Klimakrise in den Griff zu bekommen, bisher kaum sichtbar sind - während die negativen Konsequenzen immer spürbarer werden. Ich glaube, wir werden uns von dem Gedanken verabschieden müssen, dass technologische Innovationen die Lösung des Problems sein können. Wenn überhaupt, können sie nur einen Beitrag leisten. Das heißt, es braucht einen tiefgreifenden sozial-ökologischen gesellschaftlichen Wandel. Und Wissenschaft kann da einen Beitrag leisten.

SozMag: Vielen Dank für den spannenden Einblick in deine Arbeit! Wir wünschen dir gutes Gelingen bei deinen Forschungsprojekten!

\section{LITERATUR}

Brand, Ulrich/Wissen, Markus (2017): Imperiale Lebensweise: Zur Ausbeutung von Mensch und Natur im globalen Kapitalismus. München: Oekom.

de Schutter, Liesbeth/Wieland, Hans/Gözet, Burcu/ Giljum, Stefan (2017): Environmental Inequality in Europe-Towards an environmental justice framework for Austria in an EU contect. Institute for Ecological Economics, Wirtschaftsuniversität Wien.

Gassner, Andreas/Lederer, Jakob/Fellner, Johann (2020): Material stock development of the transport sector in the city of Vienna. In: Journal of Industrial Ecology, Artikelnummer 13024, https://doi.org/10.1111/jiec.13024.

Gould, Kenneth Alan/Lewis, Tammy L. (2017): Green gentrification: Urban sustainability and the struggle for environmental justice. Online verfügbar unter http://site. ebrary.com/id/11236880 (11.09.2020).

Haberl, Helmut/Fischer-Kowalski, Marina/Krausmann, Fridolin/Winiwarter, Verena (Hrsg.) (2016): Social ecology: Society-nature relations across time and space. Wiesbaden: Springer.

Haberl, Helmut/Wiedenhofer, Dominik/Erb, Karl-Heinz/ Görg, Christoph/Krausmann, Fridolin (2017): The Material Stock-Flow-Service Nexus: A New Approach for Tackling the Decoupling Conundrum. In: Sustainability Jg. 9/7, Artikelnummer 1049, https://doi.org/10.3390/su9071049.

Harvey, David (2009): Notes towards a theory of uneven geographical development. In: ders. (Hrsg.): Spaces of Global Capitalism: A Theory of Uneven Geographical Development. London/New York: Verso, S. 69-116.

Heynen, Nicolas/Kaika, Maria/Swyngedouw, Erik(Hrsg.) (2006): In the Nature of Cities: Urban political ecology and the politics of urban metabolism. New York: Routledge. 
Kalt, Gerhard/Wiedenhofer, Dominik/Görg, Christoph/ Haberl, Helmut (2019): Conceptualizing energy services: A review of energy and well-being along the Energy Service Cascade. In: Energy Research \& Social Science Jg. 53, S. 47-58, online verfügbar unter: https://zenodo.org/ record/3520538\#.XbbZbOhKiUk, https://doi.org/10.1016/j. erss.2019.02.026.

Loughran, Kevin (2020). Urban parks and urban problems: An historical perspective on green space development as a cultural fix. In: Urban Studies Jg. 57/11, S. 2321-2338, https://doi.org/10.1177/0042098018763555.

Martinez-Alier, Joan/Anguelovski, Isabelle/Bond, Patrick/Del Bene, Daniela/Demaria, Frederico/Gerber, Julien-Francois/Greyl, Lucie/Haas, Willi/Healy, Hali/ Marin-Burgos, Victoria/Ojo, Godwin/Porto, Marcelo/Rijnhout, Leida/Rodrguez-Labajos, Beatriz/Spangenberger, Joachim/Temper, Leah/Warlenius, Rikard/Yanez, Ivonne (2014): Between activism and science: Grassroots concepts for sustainability coined by Environmental Justice Organizations. In: Journal of Political Ecology Jg. 21, S. 19-60.

Mattioli, Giulio/Roberts, Cameron/Steinberger, Julia K./Brown, Andrew (2020): The political economy of car dependence: A systems of provision approach. In: Energy Research \& Social Science Jg. 66, Artikelnummer 101486, https://doi.org/10.1016/j.erss.2020.101486.

Newman, Peter/Kosonen, Leo/Kenworthy, Jeff (2016): Theory of urban fabrics: Planning the walking, transit/public transport and automobile/motor car cities for reduced car dependency. In: Town Planning Review Jg. 87/4, S. 429-458, https://doi.org/10.3828/tpr.2016.28.

O'Neill, Daniel W./Fanning, Andrew L./Lamb, William F./Steinberger, Julia K. (2018): A good life for all within planetary boundaries. In: Nature Sustainability Jg. 1/2, S. 88-95. https://doi.org/10.1038/s41893-018-0021-4.

Rueda, Salvador (2019): Superblocks for the Design of New Cities and Renovation of Existing Ones: Barcelona's Case. In: Nieuwenhuijsen, Mark/Khreis, Haneen (Hrsg.): Integrating Human Health into Urban and Transport Planning: A Framework. Wiesbaden: Springer, S. 135-153, https://doi.org/10.1007/978-3-319-74983-9_8.

Sorensen, Andre (2015): Taking path dependence seriously: An historical institutionalist research agenda in planning history. In: Planning Perspectives Jg. 30/22, https://doi.or g/10.1080/02665433.2013.874299.
Swyngedouw, Erik (2006): Metabolic urbanization: The making of cyborg cities. In: Heynen, Nicolas/Kaika, Maria/ Swyngedouw, Erik (Hrsg.): In the Nature of Cities: Urban political ecology and the Politics of urban metabolism. New York: Routledge, S. 105-121.

Unruh, Gregory C. (2000): Understanding carbon lock-in. Energy Policy Jg. 28/12, S. 817-830, https://doi.org/10.1016/ S0301-4215(00)00070-7.

Wiedmann, Thomas/Lenzen, Manfred/Keyßer, Lorenz T./Steinberger, Julia K. (2020): Scientists' warning on affluence. In: Nature Communications Jg. 11/1, Artikelnummer 3107, https://doi.org/10.1038/s41467-020-16941-y.

Zografos, Christos/Klause, Kai A./Connolly, James J.T./Anguelovski, Isabelle (2020): The everyday politics of urban transformational adaptation: Struggles for authority and the Barcelona superblock project. In: Cities Jg. 99, Artikelnummer 102613, https://doi.org/10.1016/j. cities.2020.102613.

\section{Das Interview wurde von Andreas Schulz vor- und nachbereitet und von Tonka Radisch und Cathrin Mund lektoriert.}


SCHWERPUNKT 


\title{
Grundverständnis
}

\section{nachhaltiger Entwicklung in Deutschland}

\author{
Ökologische Modernisierung oder Postwachstum
}

von Jonathan Schreiber

\begin{abstract}
In den Sozialwissenschaften konkurrieren nach Henkel (2016: 7) unterschiedliche „Grundverständnisse nachhaltiger Entwicklung" miteinander. Während in der ökologischen Modernisierung ein „Wachstum der Grenzen“ (Huber 1982: 10) durch technologische und soziale Veränderungen propagiert wird, gehen Postwachstumskonzepte von bereits erreichten Grenzen aus. Die Einstellungen der Gesellschaft gegenüber ökologischer Modernisierung und Postwachstum sind von besonderer Relevanz (vgl. Drews et al. 2018). Dennoch widmen sich nur wenige Untersuchungen gesellschaftlichen Einstellungen gegenüber diesen Grundverständnissen nachhaltiger Entwicklung. Die vorliegende Studie will zur Schließung dieser Lücke beitragen. Mit Hilfe einer repräsentativen Online-Befragung für deutschsprachige und in Deutschland lebende Internetnutzer ${ }^{\star}$ innen $(n=1212)$ wurde untersucht, inwiefern ökologische Modernisierung und Postwachstum in der deutschen Bevölkerung auf Zustimmung treffen.
\end{abstract}

Schlagwörter

Nachhaltige Entwicklung; ökologische Modernisierung; Postwachstum 


\section{Einstellungen zu Grund- verständnissen nachhaltiger Entwicklung}

Many new concepts like "green growth" [...] or "degrowth" have emerged recently, but public understandings of these are little known. (Drews et al. 2018: 271)

Das Leitbild einer nachhaltigen Entwicklung könnte der Schlüssel zur Bewältigung zahlreicher gegenwärtiger und zukünftiger Biodiversitätsverlust und menschliche Eingriffe in den Stickstoffkreislauf sein (vgl. Kropp 2019: 4; Rockström et al. 2009). Bedingt durch die Etymologie der Begriffe, Nachhaltigkeit' und , nachhaltige Entwicklung' (vgl. Grober 2010: 15; Rödel 2013: 117ff.) sowie ihre häufige und teils vage Verwendung in der Öffentlichkeit (vgl. Fischer et al. 2017: 621) kann jedoch kaum von einem allgemeingültigen Verständnis nachhaltiger Entwicklung ausgegangen werden (vgl. Lorenz 2014: 11). In den Sozialwissenschaften konkurrieren unterschiedliche „Grundverständnisse

\section{9}

Wie ist die deutsche Bevölkerung gegenüber ökologischer Modernisierung und Postwachstum eingestellt?

nachhaltiger Entwicklung" miteinander (Henkel 2016: 7). Dabei werden insbesondere das Grundverständnis der ökologischen Modernisierung und des Postwachstums diskutiert (vgl. Drews et al. 2018: 271). Während in der ökologischen Modernisierung ein Wachstum der Grenzen durch technologische und soziale Veränderungen propagiert wird, gehen Postwachstumskonzepte von bereits erreichten "Grenzen des Wachstums" aus (Huber 1982: 10).

Ebenso wie das Eingangszitat zeigt ein Blick in die Fachliteratur, dass sich nur ein geringer Anteil der Fachliteratur der Untersuchung gesellschaftlicher Einstellungen gegenüber diesen Grundverständnissen nachhaltiger Entwicklung widmet (vgl. Drews et al. 2018: 271). Insbesondere die Einstellungen der deutschen Bevölkerung zu ökologischer Modernisierung und Postwachstum sind daher weitestgehend unbekannt. Diese sind jedoch von Relevanz für den öffentlichen Diskurs (vgl. Neugebauer 2004: 10; vgl. United Nations Economic and Social Council 2014: 1; vgl. Henkel 2016: 19). Zum einen hängt die Legitimität politischer Entscheidungen von dem Ausmaß ab, in dem sie die Öffentlichkeit widerspiegeln. Zum anderen kann die Umsetzbarkeit politischer Maßnahmen an gesellschaftliche Einstellungen gebunden sein (vgl. Drews et al. 2018: 265f.). Daher fragt der vorliegende Text: Wie ist die deutsche Bevölkerung gegenüber ökologischer Modernisierung und Postwachstum eingestellt? 
Die gegenwärtige internationale und deutsche politische Ausrichtung im Hinblick auf nachhaltige Entwicklung zeigt ein Festhalten an Kernelementen einer ökologischen Modernisierung (vgl. CDU et al. 2018: 137; Brand/Niedermoser 2016: 227; Solheim 2017: v; Machin 2019: 208). Empirische Einzelbefunde weisen darauf hin, dass das Grundverständnis der ökologischen Modernisierung das bevorzugte gesellschaftliche Leitbild sein könnte (vgl. Huber 2011: 149; vgl. Drews et al. 2018: 267), während Inhalte des Postwachstums lediglich bei einer Minderheit auf Zustimmung treffen (vgl. Ančić/Domazet 2015: 470). Da eine positive Einstellung zu einem Thema auch eine erhöhte Zustimmung zu diesem bedeutet, stellt die vorliegende Forschungsarbeit die folgende Hypothese auf: Das Grundverständnis der ökologischen Modernisierung trifft in der deutschen Bevölkerung auf mehr Zustimmung als das Grundverständnis des Postwachstums.

Allgemein folgen Forschungsfrage und Hypothese dabei der umweltsoziologischen Einstellungsforschung im Verständnis der Umweltbewusstseinsforschung (vgl. Diekmann/Preisendörfer 2001: 105; vgl. Huber 2011: 81f.). Jüngere umweltsoziologische Ansätze mit einem Fokus auf soziale Praktiken werden somit nicht berücksichtig (vgl. Brand 2011; Shove 2010: 1279). Die hier eingenommene Distanzierung von praxistheoretischen Ansätzen (vgl. Hillebrandt: 2013) spiegelt sich letztlich auch im methodologischen Design der Studie wider (vgl. Jonas et al. 2017: 254). Mittels einer standardisierten Online-Befragung $(\mathrm{n}=1.212)$ soll hier der genannten Forschungsfrage und der Hypothese nachgegangen werden. Die im Fokus stehenden Daten stellen dabei einen Auszug aus einer selbst konstruierten und durchgeführten Befragung zu Einstellungen und Konsumverhalten dar, welche im Rahmen meiner Bachelorarbeit entstand. Dabei wurde die Stichprobenziehung in Kooperation mit der Statista GmbH realisiert.

Nach der vorangehenden Einführung in die Forschungsthematik soll der theoretische Hintergrund (Abschnitt 2) der Studie aufgezeigt werden. Daraufhin wird im Zuge einer Beschreibung der Methodik (Abschnitt 3) zunächst die Erhebungsmethode spezifiziert und die Operationalisierung von zentralen Variablen dargestellt. Anschließend an eine Darstellung der Ergebnisse (Abschnitt 4) wird der vorliegende Text durch Diskussion und Ausblick (Abschnitt 5) abgeschlossen.

\section{Theoretischer Hintergrund}

Nach einer allgemeinen Einführung in das Konzept der nachhaltigen Entwicklung (2.1), sollen im Folgenden die zwei Grundverständnisse nachhaltiger Entwicklung - ökologische Modernisierung (2.2) und Postwachstum (2.3) - nähere 
Betrachtung finden. Anschließend erfolgt eine Darstellung erster empirischer Befunde (2.4).

\subsection{Nachhaltige Entwicklung}

Das „ökologische Leitbild“ (Huber 2011: 155) einer nachhaltigen Entwicklung erhält seit den 1980er Jahren erhöhte Aufmerksamkeit. Endgültig im internationalen Diskurs angekommen war das Konzept wohl mit der Veröffentlichung des „Brundtland-Berichts“ (vgl. WCED 1987). Bis heute ist die dort festgehaltene Definition nachhaltiger Entwicklung als eine „Entwicklung, die die Bedürfnisse der Gegenwart befriedigt, ohne zu riskieren, daß künftige Generationen ihre eigenen Bedürfnisse nicht befriedigen können" (Hauff 1987: 46; Originaldefinition WCED 1987: 51) besonders gebräuchlich und soll auch für den vorliegenden Text gelten. Binnen kurzer Zeit nahm das Thema der nachhaltigen Entwicklung weltweit eine zentrale Position ein (vgl. UNCED 1992). Die Beschäftigung mit nachhaltiger Entwicklung bleibt dabei nicht auf den politischen Diskurs beschränkt (vgl. Huber 1995: 32). Durch das Inkrafttreten der 17 Ziele für nachhaltige Entwicklung (bekannt als die Sustainable Development Goals) und zahlreiche internationale, nationale und lokale Initiativen ist nachhaltige Entwicklung heute bereichsübergreifend eine Antwort auf zentrale Herausforderungen der Menschheit wie etwa Klimawandel, Biodiversitätsverlust, Armut, Hunger und Ungleichheit (vgl. United Nations General Assembly 2015).

Gegenwärtig werden zwei Grundverständnisse nachhaltiger Entwicklung besonderes prominent und intensiv diskutiert. Sie bieten rivalisierende Antworten auf die Frage nach der Umsetzung einer ausgeglichenen Bilanz von „Ressourcenverbrauch und Ressourcenregeneration“, welche der „Brundtland-Bericht" aufwirft (Henkel 2016: 5). Für die ökologische Modernisierung steht dabei eine effizientere und konsistentere Nutzung der Ressourcen im Vordergrund, wohingegen im Postwachstum bedarfsorientiert am Umfang des Ressourcenverbrauchs angesetzt wird (vgl. ebd.: 6f.).

\section{2 Ökologische Modernisierung}

Bereits 1975 wurde in der Bundesrepublik Deutschland der Debatte über den Fortschritt durch technologische Innovationen neuer Anschub gegeben (vgl. Hauff/ Scharpf 1975). Dabei etablierte sich mit dem Ansatz der ökologischen Modernisierung die Entwicklungsvorstellung der fundamentalen Umstrukturierung durch problemlösende, soziale und technologische Innovationen (vgl. Jänicke 1993: 17). Hinsichtlich der Frage, wie diese im Detail umzusetzen seien, brachten sich eine Vielzahl internationaler Autor ${ }^{*}$ innen 
in die Diskussion ein (vgl. Mol et al. 2014: 36). Zu diesem Diskurs können auch Konzepte wie "Green Economy“ (vgl. UNEP 2011:2) und „Green Growth“ (vgl. OECD 2011: 9) gezählt werden, die hier daher unter die ökologische Modernisierung subsummiert werden.

Die zentrale Annahme der ökologischen Modernisierung ist, „dass Ökonomie und Ökologie, Industrie und Natur, nicht zwangsläufig Gegensätze zu sein brauchen“ (Huber 2011: 143). Das Ziel ökologischer Modernisierung ist demnach ökologisch angepasstes Wirtschaftswachstum (vgl. Diekmann/Preisendörfer 2001:29). Allgemein sollen Produktionsprozesse und Produkte naturnah und Stoffstrom-optimiert transformiert werden (Konsistenz) und Ressourcen produktiver und in geringerem Umfang Einsatz finden (vgl. Fritz et al. 1995: 13). Die notwendige Einsparung von Ressourcen und Umweltbelastungen soll in der ökologischen Modernisierung insbesondere durch problemlösende technologische Innovationen möglich werden (vgl. Howes et al. 2010: 17; Huber 2011: 173ff.; Kangas 2019: 63). Diese sind ihrerseits durch „ökonomische und rechtlich-administrative Rahmenbedingungen und Methoden des Umweltmanagements" bedingt (Huber 2011: 143). Zur Schaffung der innovationsfördernden Rahmenbedingungen und zur ökologischen Modernisierung allgemein gesellen sich notwendige politische, soziale und kulturelle Voraussetzungen (vgl. Simonis 1984: 207; Prittwitz 1993: 7; vgl. Huber 2011: 143). Seit dem Ende der 1980er Jahre hat aus Sicht der ökologischen Modernisierung eine Transformation der Wirtschaft durch Einbezug von ökologischer Rationalität in Produktion und Konsum begonnen (vgl. Mol 2002: 94). Dieser Prozess ist nach Auffassung der ökologischen Modernisierung vorteilhaft für Wirtschaftswachstum und Umwelt (vgl. Kangas 2019: 63).

\subsection{Postwachstum}

Die Wurzeln der "Postwachstumskonzepte “ (Henkel 2016: 6) liegen insbesondere in wachstumskritischen Forschungsbeiträgen, allen voran in der Studie „Die Grenzen des Wachstums. Bericht des Club of Rome zur Lage der Menschheit“ (vgl. Meadows et al. 1972). Durch die in diesem Bericht angestoßenen Diskussionen trat der Begriff „Décroissance“, französisch für „Degrowth" beziehungsweise Postwachstum, zum ersten Mal in Debatten auf (vgl. Kallis et al. 2016: 17). Seitdem dauert der Diskurs an und es wird sogar von einer Postwachstums-Bewegung gesprochen (vgl. Eversberg/Schmelzer 2015: 29; vgl. Kallis et al. 2016: 19). Einerseits identifizieren Postwachstumskonzepte Kritikpunkte an den bestehenden Verhältnissen (vgl. Kallis et al. 2016: 20), genauer an Wachstum, Kapitalismus und dem Bruttoinlandsprodukt (BIP). Andererseits streben sie an, identifizierte 
Kritikpunkte durch die Etablierung einer Postwachstumsgesellschaft zu lösen.

Allen Perspektiven des Postwachstums ist Wachstumskritik gemein (vgl. Kallis et al. 2016: 20; vgl. Lorenz 2016: 9). Demnach lebt ein Teil der Menschheit über ihre ökologischen Verhältnisse und überstrapaziert so die Umwelt und sich selbst (vgl. Latouche 2009: 7f.). Da externalisierte Kosten wie Umweltschäden und menschliche Belastungen der Annahme nach so hoch liegen, dass sie bei ihrer Internalisierung das Wachstum aufheben würden, sei Wachstum unwirtschaftlich (vgl. Kallis et al. 2016: 23). Wachstum sei ungerecht, da Gewinne des Wachstums den Mächtigen zukommen, während marginalisierte Bevölkerungsgruppen die Kosten tragen müssen (vgl. Latouche 2009: 7f; vgl. Kallis et al. 2016: 24). Zudem wird angenommen, dass Wachstumsstrategien im Sinne der ökologischen Modernisierung keine Entlastung für die Umwelt und das Klima bringen, da Effizienzgewinne durch sogenannte Rebound-Effekte kompensiert werden (vgl. Paech 2012: 17). Damit ist gemeint, dass etwaige Einsparungen durch damit einhergehende erhöhte Produktion oder Konsumption aufgehoben werden können. Die Kritik am Wachstum ist eng verbunden mit jener am Kapitalismus, da dieser als zentraler Antrieb des Wachstums gilt (vgl. Andreucci/McDonough 2016: 148). Das BIP ist aus Sicht des Postwachstums ein unzureichender Indikator für gesellschaftlichen Wohlstand, denn Verteilungsungleichheiten werden kaum berücksichtigt (vgl. Paech 2012: 17). Darüber hinaus kann BIP-Wachstum durch viele nicht gemeinwohlfördernde Ursachen zustande kommen (vgl. Victor 2016: 191).

Ein Teil der Postwachstumsliteratur widmet sich der Konstruktion eines alternativen Gesellschaftsentwurfs (vgl. Seidl/ Zahrnt 2010). Dem Gestaltungsprinzip der Suffizienz, welches vor allem Reduktion und Entschleunigung beinhaltet, kommt dabei allgemein eine wichtige Rolle $\mathrm{zu}$ (vgl. Paech 2012: 18). Serge Latouche (2009: 32), identifiziert acht konkrete Ziele, die sogenannten „eight ,R's“, die zu einer Postwachstumsgesellschaft führen können. Da ich einen Teil dieser Ziele genutzt habe, um die Einstellung gegenüber Postwachstum abzufragen, wird hier näher auf die „eight ,R`s“, eingegangen. Mit (1) „Restructure" wird auf eine Umstrukturierung des Produktionsapparats und der sozialen Beziehungen abgezielt (Latouche 2009: 36). Die Überwindung des Wachstums und das Hinwenden zu einer ",anderen Logik“ sind demnach essenziell (Latouche 2009: 8). Die Umweltbelastung durch Umstrukturierung von Produktion und Konsum zu minimieren, ist das Anliegen von (2) „Reduce“ (ebd.: 38) und damit „to do with less in terms of energy and material products" (Ančić/Domazet 2015: 457). Dem Postwachstum und (3) „Reconceptualize“ nach sollen Werteverständnisse und damit 
Konzepte wie Wohlstand und Armut neu definiert und verstanden werden. Darüber hinaus sollen mit (4) „Re-evaluate“ auch neue Werte wie Verantwortung und Mäßigung etabliert werden. Unter dem Stichwort (5) „Redistribute“ wird Verteilungs-, Umwelt- und Klimagerechtigkeit gefordert. Mit (6) „Relocalize“ möchte Postwachstum Wirtschaft, Produktion und Konsum wieder auf die lokale Ebene verlagern. Schließlich zielt Postwachstum mit (7) „Re-use“ und (8) „Recycle“ darauf $\mathrm{ab}$, die Lebensdauer von Produkten zu verlängern sowie den anfallenden Müll zu reduzieren und vollständig zu recyclen (Latouche 2009: 34ff.).

\section{4 Ökologische Modernisierung und Postwachstum in der Gesellschaft}

Im Folgenden werden bestehende Untersuchungen aufgezeigt, welche gesellschaftliche Wahrnehmungen und Einstellungen zu ökologischer Modernisierung und Postwachstum näher untersuchen. Aufgrund kaum vorhandener, themenspezifischer deutscher Studien werden dabei insbesondere internationale Befunde aufgegriffen und auch Gruppierungen innerhalb der Bevölkerung in den Blick genommen.

So strebten Niklas Harring et al. (vgl. 2011: 389) an, Ursachen für Schwankungen im Umweltbewusstsein der schwedischen
Bevölkerung seit den 1980er Jahren zu identifizieren. Dabei wurde insbesondere der Diskurseintritt der ökologischen Modernisierung herangezogen. Besonders relevant ist demnach die Frage, inwiefern ökologische Modernisierung in anderen Ländern wie Deutschland an Bedeutung gewonnen hat und die öffentliche Wahrnehmung beeinflusst (vgl. Harring et al. 2011: 398f.). Aktuelle Bevölkerungsbefragungen deuten heute zumindest darauf hin, dass Elemente einer ökologischen Modernisierung, im deutschsprachigen Raum auf Zustimmung treffen. $\mathrm{Zu}$ nennen sind hierbei insbesondere die Annahme der Wirtschaftsförderung sowie Wohlstandssicherung durch Umweltschutz (vgl. Schwegler et al. 2015: 10; vgl. BMUB/ UBA 2017: 20). Auf Basis einer quantitativen Untersuchung von 91 Artikeln zum Thema Degrowth gelangten Martin Weiss und Claudio Cattaneo (vgl. 2017: 225) zu dem Ergebnis, dass es das Ziel zukünftiger Forschung sein sollte, die gesellschaftliche Wahrnehmung von Postwachstum empirisch zu untersuchen. Mit Hilfe einer repräsentativen Online-Befragung zeigten Stefan Drews und Jeroen van den Bergh (vgl. 2016: 2), dass nur 15 Prozent der spanischen Bevölkerung die Verfolgung des Wirtschaftswachstums aufgeben wollen (Postwachstum). Demgegenüber sprachen sich 59 Prozent der Befragten für eine ökologische Modernisierung aus (vgl. Drews/van den Bergh 2016: 7). In einer weiteren Studie wurde untersucht, 
inwiefern Postwachstum und ökologische Modernisierung unter Wissenschaftler* innen auf Zustimmung treffen (vgl. Drews/ van den Bergh 2017). In einem Vergleich mit der spanischen Gesamtbevölkerung (vgl. Drews et al. 2019) konnten drei unterschiedliche Ansichten zu Wachstum und Umwelt unter Wissenschaftler*innen (ökologische Modernisierung, Postwachstum, Wachstumsignoranz) und die gleichen Ansichten sowie eine zusätzliche (Gleichgültigkeit) in der Bevölkerung identifiziert werden (vgl. ebd.: 145ff.). Die empirischen Befunde deuten darauf hin, dass Wissenschaftler*innen wesentlich konsistentere Einstellungen zu den Grundverständnissen nachhaltiger Entwicklung aufweisen, als die Bevölkerung. Innerhalb der Bevölkerung scheint es demnach möglich zu sein, sowohl gegenüber der ökologischen Modernisierung als auch gegenüber dem Postwachstum positiv eingestellt zu sein eine Ambivalenz, die auch in der Operationalisierung der vorliegenden Untersuchung berücksichtigt wird (vgl. ebd.: 150).

Auf internationaler Betrachtungsebene konnte in einer weiteren Studie gezeigt werden, dass die Mehrheit der Bevölkerung der Vereinigten Staaten und der Europäischen Union Wirtschaftswachstum und Umweltschutz als miteinander kompatibel ansieht (vgl. Drews et al. 2018: 267). Branko Ančić und Mladen Domazet (vgl. 2015: 459), kamen zu dem Ergebnis, dass sowohl in ärmeren als auch in reicheren

Ländern der Europäischen Union kleine Bevölkerungsteile mit Postwachstum sympathisieren. Schließlich wurde im Rahmen der vierten internationalen Degrowth-Konferenz im Jahr 2014 eine erste Befragung der wachstumskritischen Bewegung durchgeführt. Dabei gelang es, weitestgehend konsensuale Positionen der Befragten zu identifizieren und damit empirische Belege für das Entstehen einer „Degrowth-Bewegung" zu sammeln (vgl. Eversberg/Schmelzer 2015: 7, 29).

\section{Methodisches Vorgehen}

Mit dem Forschungsanliegen der vorliegenden Arbeit wird nach ,verallgemeinerbaren Aussagen" gesucht (Häder 2015: 13). Eine solche statistische Generalisierbarkeit auf eine festgelegte Grundgesamtheit lässt sich mit Hilfe von standardisierten quantitativen Befragungen erreichen (vgl. Brosius et al. 2009: 113; vgl. Baur/Blasius 2019: 11). So wurde eine Online-Befragung durchgeführt, bei der die Grundgesamtheit „deutsche Bevölkerung " als deutschsprachige, volljährige Internetnutzer*innen verstanden wird, die gegenwärtig in Deutschland leben. Obgleich unterschiedliche Studien existieren, die sich mit Nachhaltigkeit und damit in Verbindung stehenden Einstellungen befassen (vgl. Schwegler et al. 2015: 10; vgl. BMUB/ UBA 2017: 20), gab es kein Messinstrument, welches für die angestrebte Befragung vollständig übertragbar gewesen wäre. Daher 
habe ich einen Fragebogen konstruiert, welcher 18 Items zu ökologischer Modernisierung und Postwachstum umfasst. Jedes Item kann dabei fünf unterschiedliche Ausprägungen annehmen - von $1=$ „stimme überhaupt nicht zu“ bis $5=$ „stimme voll und ganz zu“. Um Reihenfolgeeffekte zu minimieren wurden die Fragebatterien randomisiert und in vier (a bis d) Blöcke aufgeteilt: (a) vier randomisierte Items zur ökologischen Modernisierung, (b) fünf randomisierte Items zum Postwachstum, (c) fünf randomisierte Items zur ökologischen Modernisierung und (d) vier randomisierte Items zum Postwachstum. Im Zuge der Hypothesentestung wurde nachfolgend jeweils ein Index (aus jeweiligen Items aufsummierter Score) für die Einstellung gegenüber ökologischer Modernisierung und Postwachstum gebildet und ein Mittelwertvergleich der beiden Indexe durchgeführt. Somit war ein Vergleich der jeweiligen $\mathrm{Zu}$ stimmung zu beiden Grundverständnissen nachhaltiger Entwicklung möglich.

Aus Theorie und bestehender Forschung wurden neun Items extrahiert, um Einstellungen gegenüber ökologischer Modernisierung als Grundverständnis von nachhaltiger Entwicklung abzufragen. Diese sind in einen Index für ökologische Modernisierung (IndexÖko) eingegangen (siehe Tabelle 2, Anhang). Im Rahmen der Befragung wurden Teilnehmende dazu aufgefordert, auf einer fünfstufigen Likert-Skala ihre Zustimmung zu den Aussagen anzugeben. Analog zur Vorgehensweise bei der Erstellung des IndexÖko flossen auch in den Index für Postwachstum (IndexPost) aus Theorie und bestehender Forschung neun Items ein (siehe Tabelle 3, Anhang). Diese Items orientieren sich dabei unter anderem an sechs der „eight ,R's“: „Re-evaluate“, „Reconceptualize“, „Restructure“, „Redistribute“, „Relocalize“ und „Reduce“ (Latouche 2009: 33ff.). Ein solches Vorgehen erwies sich in bereits bestehender Forschung als sinnvoll (vgl. Ančić/Domazet 2015: 459).

Um auf die Grundgesamtheit übertragbare Ergebnisse bieten zu können, ist die Berücksichtigung der ,soziale[n] Basis von Umwelteinstellungen essenziell (vgl. Diekmann/Preisendörfer 2001: 109). Innerhalb der vorliegenden Befragung wurden daher auf demographischer Ebene Alter, Geschlecht, Bildungsabschluss, Einkommen und Wohnort der Stichprobe untersucht (vgl. Li et al. 2019: 30). Zudem wurden die politische Einstellung (LinksRechts-Skala) und die Anerkennung des Klimawandels erhoben.

\section{Ergebnisse}

Im folgenden Teil wird in einem ersten Abschnitt auf die Stichprobe der Untersuchung eingegangen (4.1). Im Anschluss werden die Ergebnisse der Hypothesenauswertung dargestellt (4.2). Das Signifikanzniveau wurde auf $p<0,05$ gesetzt. 


\subsection{Die Stichprobe}

Die Stichprobe der Befragung wurde nach Alter und Geschlecht kreuzquotiert. Dabei zielte die Quotierung auf eine Repräsentativität für volljährige, deutschsprachige und in Deutschland lebende Internetnutzerinnen ab. Die Quotierung wurde vom Kooperationspartner Statista $\mathrm{GmbH}$ übernommen, welcher durch regelmäßige Projekte und Austausch mit Partner*innen die Aktualität der zugrunde liegenden Werte absichert. Die Erhebung fand vom 23. Mai bis zum 5. Juni 2019 statt und umfasste somit auch den Zeitraum der Direktwahlen zum Europäischen Parlament. Aufgrund der damit verbundenen Präsenz von Themen wie Umweltschutz, Klimawandel und Sozialstandards kann zum Erhebungszeitraum von einer stärkeren Informiertheit der Befragten zu diesen, auch nachhaltige Entwicklung betreffenden Themen ausgegangen werden.

Insgesamt beantworteten 1.494 Teilnehmende den Fragebogen vollständig, davon wurden 1.212 Fälle in der Auswertung berücksichtigt (siehe Tabelle 1). Trotz der verminderten Fallzahl liegt die Merkmalsverteilung für Alter und Geschlecht sehr nah an der ursprünglichen Quotierung und an den Ergebnissen bisheriger Studien zu demographischen Merkmalen deutscher Internetnutzer*innen (vgl. Frees/Koch 2018: 400). Das Durchschnittsalter der Befragten lag bei 45,58 Jahren, mit einer
Standardabweichung $(\sigma)$ von 15,3 . Die Analysestichprobe $(\mathrm{n}=1.212)$ setzte sich aus 50,41 Prozent weiblichen und 49,59 Prozent männlichen Befragten zusammen. Der häufigste höchste Bildungsabschluss in der Stichprobe war mit 39,44 Prozent der Befragten die Berufsausbildung. Das Haushaltseinkommen der Befragten nach Abzug von Beiträgen zur Renten-, Arbeitslosen- und Krankenversicherung wurde kategorial erhoben. Rund zwei Fünftel der Befragten verdienten dabei zwischen 1500 und 3000 Euro. Der Median (med) der Links-Rechts-Skala lag bei 5 und bildete gleichzeitig den Modalwert (37,13 Prozent der Fälle; $n=1.212$ ). Obwohl eine Kenntnis des Wortes „Nachhaltigkeit“ keine direkte Voraussetzung für Einstellungen zu ökologischer Modernisierung und Postwachstum ist, kann festgehalten werden, dass 94,14 Prozent der Befragten das Wort kannten. Rund ein Prozent der Befragten war der Ansicht, dass sich das globale Klima gegenwärtig nicht verändert. Ein Basiswissen über ökonomische Zusammenhänge ist in der Stichprobe gegeben, denn mehr als zwei Drittel der Befragten (70,13 Prozent) konnten das BIP als korrekten Indikator für das Wirtschaftswachstum identifizieren. 


\begin{tabular}{|c|c|c|}
\hline Variable & Ausprägungen & Anteil in Prozent \\
\hline Alter & & $\begin{array}{l}\mathrm{m}=45.58 \\
(\sigma=15,3)\end{array}$ \\
\hline Alter (kategorisiert) & $\begin{array}{l}18 \text { bis } 29 \text { Jahre } \\
30 \text { bis } 49 \text { Jahre } \\
50 \text { Jahre und älter }\end{array}$ & $\begin{array}{l}18,89 \\
39,60 \\
41,50\end{array}$ \\
\hline Geschlecht & $\begin{array}{l}\text { weiblich } \\
\text { männlich }\end{array}$ & $\begin{array}{l}50,41 \\
49,59\end{array}$ \\
\hline Höchster Bildungsabschluss & $\begin{array}{l}\text { (noch) kein allgemeiner Schulabschluss } \\
\text { Hauptschul-/Volksschulabschluss } \\
\text { Realschulabschluss/mittlere Reife } \\
\text { Abitur } \\
\text { Berufsausbildung (Geselle/Meister) } \\
\text { Bachelorabschluss oder Gleichwertiges } \\
\text { Masterabschluss oder Gleichwertiges } \\
\text { Doktortitel oder Gleichwertiges } \\
\end{array}$ & $\begin{array}{l}0,83 \\
6,52 \\
16,25 \\
14,44 \\
39,44 \\
9,65 \\
11,55 \\
1,32 \\
\end{array}$ \\
\hline $\begin{array}{l}\text { Durchschnittliches monatliches Haus- } \\
\text { haltsnettoeinkommen }\end{array}$ & $\begin{array}{l}\text { bis unter } 1.500 € \\
1.500 € \text { bis unter } 3.000 € \\
3.000 € \text { und mehr } \\
\text { möchte ich nicht beantworten } \\
\end{array}$ & $\begin{array}{l}25,17 \\
42,24 \\
25,00 \\
7,59 \\
\end{array}$ \\
\hline Familienstand & $\begin{array}{l}\text { ledig } \\
\text { in einer Beziehung } \\
\text { verheiratet } \\
\text { geschieden/verwitwet } \\
\text { keine Angabe }\end{array}$ & \begin{tabular}{l|}
27,39 \\
18,23 \\
41,67 \\
11,80 \\
0,91 \\
\end{tabular} \\
\hline Größe des Wohnorts & $\begin{array}{l}\text { Landgemeinde } \\
\text { Kleinstadt } \\
\text { Mittelstadt } \\
\text { Großstadt } \\
\text { Millionenstadt } \\
\text { weiß nicht }\end{array}$ & $\begin{array}{l}17,5 \\
20,5 \\
25,1 \\
24,0 \\
11,2 \\
1,7\end{array}$ \\
\hline Links-Rechts-Skala & 1 (links) bis 10 (rechts) & $\begin{array}{l}\operatorname{med}=5 ; M=5,01 \\
(\sigma=1,75)\end{array}$ \\
\hline $\begin{array}{l}\text { Schon einmal von "Nachhaltigkeit“ } \\
\text { gehört? }\end{array}$ & \begin{tabular}{|l} 
Ja \\
Nein \\
\end{tabular} & \begin{tabular}{|l|}
94,14 \\
5,86 \\
\end{tabular} \\
\hline Klimawandel & $\begin{array}{l}\text { Das globale Klima verändert sich... } \\
\ldots \text { eindeutig } \\
\ldots \text { wahrscheinlich } \\
\ldots \text { wahrscheinlich nicht } \\
\ldots \text { definitiv nicht } \\
\text { weiß nicht }\end{array}$ & $\begin{array}{l}70,79 \\
22,19 \\
2,97 \\
1,07 \\
2,97 \\
\end{array}$ \\
\hline $\begin{array}{l}\text { "Mit welchem der folgenden Indi- } \\
\text { katoren wird Ihrer Ansicht nach das } \\
\text { Wirtschaftswachstum gemessen?" }\end{array}$ & $\begin{array}{l}\text { Geldmenge } \\
\text { Erzeugerindex (PPI) } \\
\text { Zahlungsbilanz } \\
\text { Bruttoinlandsprodukt (BIP) } \\
\text { weiß nicht }\end{array}$ & $\begin{array}{l}5,78 \\
4,21 \\
4,13 \\
70,13 \\
15,76\end{array}$ \\
\hline
\end{tabular}

Tabelle 1: Deskriptive Beschreibung der Stichprobe (eigene Darstellung) 


\subsection{Vergleich der Indizes für Postwachstum und ökologische Modernisierung}

In einem ersten Schritt bildete ich additive Indexe für ökologische Modernisierung und Postwachstum aus den dazugehörigen Items. Einen Überblick über die Durchschnittswerte der einzelnen Items bietet Abbildung 1. Jeweils ein Item pro Index war negativ gepolt und wurde vor der Indexbildung umgepolt. Vor der Hypothesentestung bewertete ich die Qualität
Reliabilitätsanalyse der Items sowie konfirmatorischer Faktorenanalyse. Die Untersuchungen zur Trennschärfe der Items des IndexÖko und IndexPost führten zum Ausschluss der beiden umgepolten negativ gepolten Items. Da alle anderen Items eine Trennschärfe von mindestens 0,2 aufwiesen und somit beibehalten werden konnten (vgl. Möltner et al. 2006: 5), verminderte sich die Anzahl der Items pro Index auf acht. Die Reliabilitätsanalyse des IndexÖko wurde mit Cronbachs Alpha ( $a$ ) durchgeführt und ergab einen akzeptablen Wert $(\alpha=0,71)$. Etwas höher lag Cronbachs

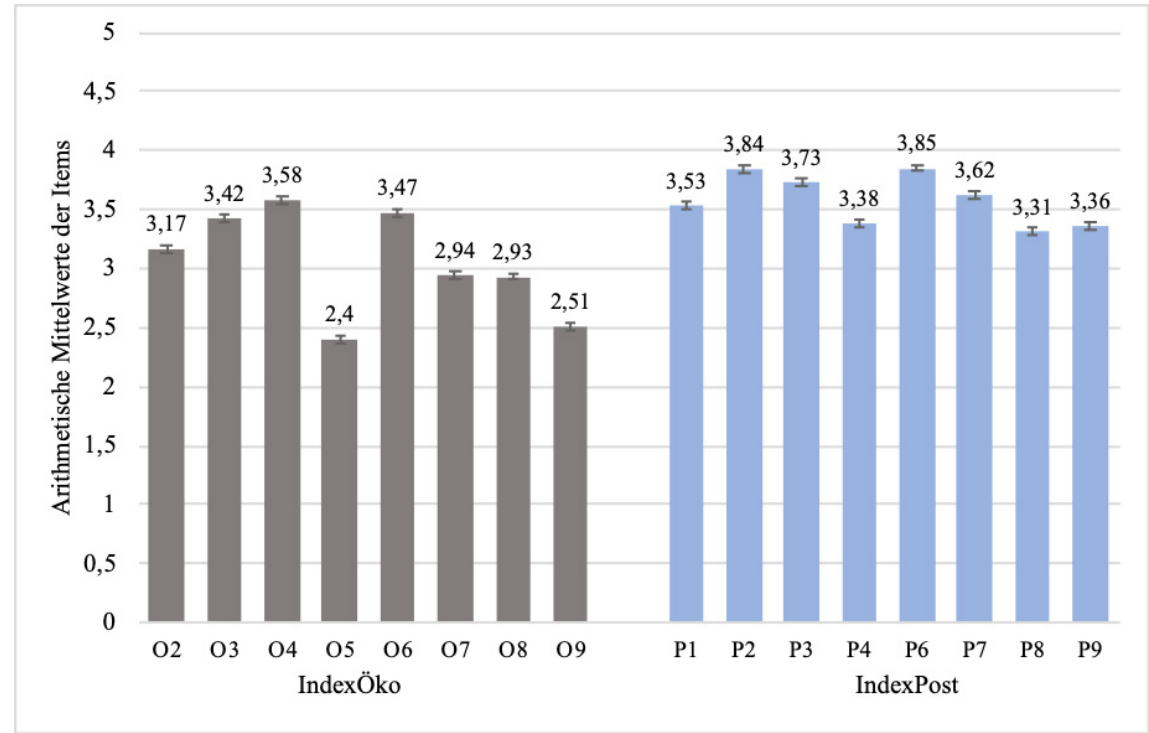

Anmerkungen: Für Erläuterungen zu den Items siehe Tabelle 2 und 3 im Anhang. Fehlerbalken zeigen Standardfehler. Minimum (= 1, ,stimme überhaupt nicht $\left.\mathrm{zu}^{\prime \prime}\right)$ und Maximum (= 5, „stimme voll und ganz $\mathrm{zu}$ “) sind für alle Items gleich. $\mathrm{n}=1.212$.

Abbildung 1: Mittelwerte der Items der Indexe für ökologische Modernisierung (IndexÖko) und Postwachstum (IndexPost) im Vergleich 
Alpha für den IndexPost $(\alpha=0,85)$. Bei der Faktorenanalyse wurden hohe Faktorladungen generell mit 0,3 begrenzt, darunter liegende Items sollten eliminiert werden. Bei Anwendung des Kaiser-Kriteriums ergab sich für den IndexÖko, dass alle Items auf einen Faktor mit einem Eigenwert (EW) in Höhe von 2 laden (vgl. Kaiser/Dickman 1959). Da aus der Stichprobe gezogene Faktoren auch durch Zufall einen EW aufweisen können, der über 1 liegt, wurden zudem ein Screeplot und Horns Parallelanalyse (100 Datensätze generiert) durchgeführt (vgl. Moosbrugger/Schermelleh-Engel 2012: 331). Die Ergebnisse wiesen darauf hin, dass der Index für ökologische Modernisierung sogar auf einen zweiten Faktor lädt, welcher jedoch nach dem hier angewendeten
Kaiser-Kriterium eliminiert wurde (EW $=0,83)$. Eine Überschätzung des einen Faktors war auf Basis der Parallelanalyse zudem unwahrscheinlich. Der Faktor erklärte zwischen 15,03 und 32,88 Prozent der Varianz der Items des Indexes. Unter Anwendung des Kaiser-Kriteriums lädt auch der IndexPost auf einen Faktor (EW $=3,34)$. Bei weiterer Scree- und Parallelanalyse erhärtete sich dieses Ergebnis. Der Faktor konnte zwischen 27,98 und 53,78 Prozent der Varianz der Items des Indexes erklären. Beide Indexe konnten sich in der Qualitätsprüfung behaupten und gingen somit in ihrer neuen Form mit jeweils acht Items in die Analyse ein.

Die Hypothese prognostiziert einen signifikanten Unterschied in der Stärke

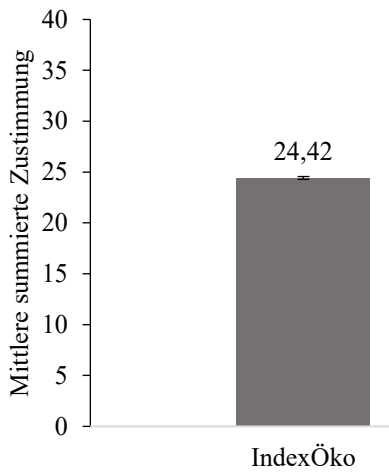

28,62

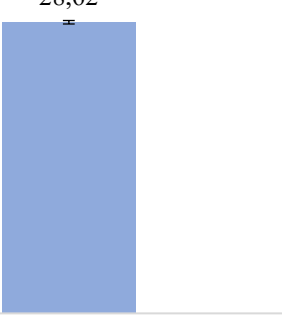

IndexPost

Anmerkungen: Signifikante $\left({ }^{\star} \mathrm{p}<0,05\right)$ Mittelwertdifferenz der Indexe für ökologische Modernisierung (IndexÖko) und Postwachstum (IndexPost). Maximal erreichbarer Wert pro Index $=40$. Fehlerbalken zeigen Standardfehler (Std. FehlerIndexÖko = 0,14; Std. FehlerIndexPost $=0,17 ; \mathrm{n}=1.212$ ).

Abbildung 2: Einstellung gegenüber Grundverständnissen nachhaltiger Entwicklung in der deutschen Bevölkerung 
der Zustimmung zum Grundverständnis der ökologischen Modernisierung und jenem des Postwachstums. Dabei wurde angenommen, dass die deutsche Bevölkerung gegenüber dem Grundverständnis der ökologischen Modernisierung positiver eingestellt ist als gegenüber dem Grundverständnis des Postwachstums. Somit sollten Mittelwerte von zwei unterschiedlichen Indizes miteinander verglichen und Unterschiede auf Signifikanz getestet werden. Zur Überprüfung der Hypothese führte ich ein Mittelwertvergleich der Indexe für Postwachstum und ökologische Modernisierung mit einem Zweistichproben-t-Test durch (siehe Abbildung 2). Der t-Test zeigte, dass sich die Mittelwerte $(m)$ des IndexÖko $\left(\mathrm{m}_{\text {IndexÖko }}=\right.$ 24,43; Std. Abw. = 4,97; 95\%-Konfidenzintervall $[24,15 ; 24,71])$ und des IndexPost $\left(\mathrm{m}_{\text {IndexPost }}=28,29 ;\right.$ Std. $A b w .=5,99$, 95\%-Konfidenzintervall $[28,29 ; 28,96]$ ) signifikant voneinander unterscheiden $(t(1211)=-22,55 ; p<0,05)$. Dabei beträgt die Mittelwertdifferenz $-4,2$.

\section{Diskussion und Ausblick}

Ausgangspunkt der vorliegenden Untersuchung ist die gegenwärtige wissenschaftliche Auseinandersetzung mit den zwei Grundverständnissen nachhaltiger Entwicklung - ökologische Modernisierung und Postwachstum. Erste Einzelbefunde legten nahe, dass sich mit diesen Grund- verständnissen verbundene Einstellungen auch in Teilen der deutschen Bevölkerung wiederfinden (vgl. Ančić/Domazet 2015: 470; vgl. BMUB/UBA 2017: 20; vgl. Drews et al. 2018: 268). Daraus ergab sich das Forschungsanliegen zu untersuchen, wie die deutsche Bevölkerung gegenüber ökologischer Modernisierung und Postwachstums eingestellt ist. Ausgehend von der momentanen internationalen und deutschen politischen Ausrichtung sowie empirischen Befunden (vgl. Drews/van den Bergh 2016: 7; vgl. Drews et al. 2018: 267) wurde eine Hypothese formuliert. Darin wird vermutet, dass die deutsche Bevölkerung dem Grundverständnis der ökologischen Modernisierung mehr zustimmt als dem Grundverständnis des Postwachstums. Hierbei kann kritisch angemerkt werden, dass mit dem Forschungsanliegen eine auf das Individuum fokussierte Perspektive der umweltsoziologischen Einstellungsforschung eingenommen wurde (vgl. Diekmann/Preisendörfer 2001: 105; vgl. Huber 2011: 81f.). Ein Blickwinkel auf Postwachstum und ökologische Modernisierung in der deutschen Bevölkerung, welcher auch die Lebensrealität und das soziale Umfeld der Menschen betrachtet, könnte perspektivisch durch praxistheoretische Untersuchung ermöglicht werden (vgl. Hillebrandt: 370 ).

Die Reliabilitätsprüfung von IndexÖko und IndexPost ergab jeweils ein zufriedenstellendes Ergebnis. Die für die Indexkon- 


\section{Die Hypothesenprüfung zeigt, dass das Grundverständnis des Postwachstums in der deutschen Bevölkerung auf signifikant stärkere Zustimmung trifft als das der ökologischen Modernisierung.}

struktion verwendeten Items und ihre Nähe zum Forschungsstand sowie das Forschungsdesign legen zunächst valide Ergebnisse nahe. Reflektierend muss jedoch angemerkt werden, dass die Items des IndexÖko kontrastreicher ausfielen als jene des IndexPost. So wurden mit dem IndexÖko etwa Einstellungen zu technologischer Innovation abgefragt, welche nicht zwangsläufig mit Aspekten der ökologischen Modernisierung verbunden sein müssen (siehe Item O8, Tabelle 2, Anhang). Vor diesem Hintergrund ist eine mögliche Beeinträchtigung der Qualität und Vergleichbarkeit der Indexe nicht auszuschließen.

Die Hypothesenprüfung zeigt, dass das Grundverständnis des Postwachstums in der deutschen Bevölkerung auf signifikant stärkere Zustimmung trifft als das der ökologischen Modernisierung. Die Hypothese kann daher nicht beibehalten werden. Dieses Resultat weicht von Befunden $a b$ nach denen einzelne Inhalte einer ökologischen Modernisierung in der deutschen Bevölkerung auf mehr Zustimmung treffen als solche des Postwachstums (vgl. Ančić/Domazet 2015: 470; vgl. Drews et al. 2018: 267). Andere Einzelbefunde lassen hingegen eine Zustimmung zu Inhalten des Postwachstums in der deutschen Bevölkerung naheliegend erscheinen (vgl. BMUB/UBA 2017: 20).

Eine mögliche Erklärung für die starke Bedeutung von Postwachstum in der deutschen Bevölkerung könnte in den Effekten sozialer Bewegungen - insbesondere der „Degrowth-Bewegung“ (Eversberg/ Schmelzer 2015: 29) sowie der Klimabewegung (vgl. Kössler 2013) - und den von ihnen angestoßenen Debatten liegen (vgl. Brand/Krams 2018: 21). Darüber hinaus wäre auch ein allgemeiner Einstellungswandel in der Bevölkerung denkbar, welcher auf andere Ursachen wie die Bedrohung durch den Klimawandel (vgl. United Nations 2016: 1) oder Debatten rund um die Europawahl 2019 zurückzuführen ist. Beide Optionen könnten in weiterführenden Untersuchungen verfolgt werden.

Fraglich bleibt darüber hinaus, inwiefern die Bundesregierung die Wichtigkeit von Postwachstum für die deutsche Bevölkerung in ihren Entscheidungen berücksichtigt. Die COVID-19-Pandemie hat 
zu einem Einbruch der wirtschaftlichen Leistung in Deutschland geführt - ein Umstand, dem die Bundesregierung mit einem Konjunkturprogramm in Höhe von 130 Milliarden Euro entgegenwirken möchte (vgl. Bundesministerium der Finanzen 2020). Dabei lässt sich feststellen, dass mit dem Konjunkturprogramm insbesondere Nachhaltigkeitsaspekte im Sinne der ökologischen Modernisierung Berücksichtigung finden. Ein Hinwenden zu einer Postwachstumseinstellung ist hingegen kaum zu erkennen. Allgemein scheint es, als ob Postwachstum im politischen Diskurs um die Auswirkungen der COVID-19-Pandemie nur eine geringe Rolle spielt. Eine Untersuchung dieser Vermutung wäre vor dem Hintergrund der in dieser Studie gefundenen Einstellungen der deutschen Bevölkerung und der damit verbundenen Legitimität politischer Entscheidungen von besonderem Interesse.

\section{LITERATUR}

Ančić, Branko/Domazet, Mladen (2015): Potential for Degrowth: Attitudes and behaviours accross 18 European countries. In: Teorija in Praksa Jg. 52/3, S. 456-475.

Andreucci, Diego/McDonough, Terrence (2016): Kapitalismus. In: D‘Alisa, Giacoma/Demaria, Federico/Kallis, Giorgios (Hrsg.): Degrowth: Handbuch für eine neue Ära. München: oekom, S. 147-151.

Baur, Nina/Blasius, Jörg (2019): Methoden der empirischen Sozialforschung - Ein Überblick. In: dies. (Hrsg.): Handbuch Methoden der empirischen Sozialforschung. Wiesbaden: Springer VS, S. 1-28.

Belitz, Heike/Kirn, Tanja (2008): Deutlicher Zusammenhang zwischen Innovationsfähigkeit und Einstellungen zu
Wissenschaft und Technik im internationalen Vergleich. In: Vierteljahrshefte zur Wirtschaftsforschung Jg. 77/2, S. 47-64.

BMUB/UBA (2017): Umweltbewusstsein in Deutschland 2016: Ergebnisse einer repräsentativen Bevölkerungsumfrage. Dessau-Roßlau.

Brand, Karl-Werner (2011): Umweltsoziologie und der praxistheoretische Zugang. In: Groß, Matthias (Hrsg.): Handbuch Umweltsoziologie. Wiesbaden: Springer VS, S. 173-198.

Brand, Ulrich/Krams, Mathias (2018): Zehn Jahre Degrowth als radikale politische Perspektive: Potenziale und Hürden. In: Forschungsjournal Soziale Bewegungen Jg. 31/4, S. 18-26.

Brand, Ulrich/Niedermoser, Katrin (2016): Gewerkschaften zwischen "ökologischer Modernisierung" und Einsatzpunkten sozial-ökologischer Transformation. In: Barth, Thomas/ Jochum, Georg/Littig, Beate (Hrsg.) Nachhaltige Arbeit: Soziologische Beiträge zur Neubestimmung der gesellschaftlichen Naturverhältnisse. Frankfurt am Main: Campus, S. 223-243.

Brosius, Hans-Bernd/Koschel, Friederike/Haas, Alexander (2009): Methoden der empirischen Kommunikationsforschung: Eine Einführung. Wiesbaden: Springer VS.

Bundesministerium der Finanzen (BMF) (2020): Corona-Folgen bekämpfen, Wohlstand sichern, Zukunftsfähigkeit stärken, Ergebnis Koalitionsausschuss 3. Juni.

CDU/CSU/SPD (2018): Ein neuer Aufbruch für Europa Eine neue Dynamik für Deutschland Ein neuer Zusammenhalt für unser Land: Koalitionsvertrag zwischen CDU, CSU und SPD. Berlin.

Diekmann, Andreas/Preisendörfer, Peter (2001): Umweltsoziologie: Eine Einführung. Reinbek bei Hamburg: Rowohlt.

Drews, Stefan/Antal, Miklós/van den Bergh, Jeroen C.J.M. (2018): Challenges in Assessing Public Opinion on Economic Growth Versus Environment: Considering European and US Data. In: Ecological Economics Jg. 146, S. 265-272.

Drews, Stefan/Savin, Ivan/van den Bergh, Jeroen C.J.M. (2019): Opinion Clusters in Academic and Public Debates on Growth-vs-Environment. In: Ecological Economics Jg. 157, S. 141-155.

Drews, Stefan/van den Bergh, Jeroen C.J.M. (2016): Public views on economic growth, the environment and 
prosperity: Results of a questionnaire survey. In: Global Environmental Change Jg. 39, S. 1-14.

Drews, Stefan/van den Bergh, Jeroen C.J.M. (2017): Scientists' views on economic growth versus the environment: a questionnaire survey among economists and non-economists. In: Global Environmental Change Jg. 46, S. 88-103.

Eversberg, Dennis/Schmelzer, Matthias (2015): Degrowth: Kapitalismuskritik muss praktisch werden! Grundkonsens und Differenzen einer entstehenden Bewegung. Working Paper 1/2015 der DFG-KollegforscherInnengruppe Postwachstumsgesellschaften.

Fischer, Daniel/Haucke, Franziska/Sundermann, Anna (2017): What does the Media mean by 'sustainability' or 'sustainable development'? An empirical analysis of sustainability terminology in German newspapers over two decades. In: Sustainable Development Jg. 25/6, S. 610-624.

Fisher, Dana R./Freudenburg, William R. (2001): Ecological Modernization and Its Critics: Assessing the Past and Looking Toward the Future. In: Society \& Natural Resources Jg. 14/8, S. 701-709.

Frees, Beate/Koch, Wolfgang (2018): ARD/ZDF-Onlinestudie 2018: Zuwachs bei medialer Internetnutzung und Kommunikation. In: Media Perspektiven Jg. 2018/9, S. 398-413.

Fritz, Peter/Huber, Joseph/Levi, Hans Wolfgang (1995): Das Konzept der nachhaltigen Entwicklung als neue Etappe der Suche nach einem umweltverträglichen Entwicklungsmodell der modernen Gesellschaft: Einleitung und Überblick zu den Beiträgen des Bandes. In: dies. (Hrsg.): Nachhaltigkeit in naturwissenschaftlicher und sozialwissenschaftlicher Perspektive. Stuttgart: Hirzel, S. 7-16.

Grober, Ulrich (2010): Die Entdeckung der Nachhaltigkeit: Kulturgeschichte eines Begriffs. München: Kunstmann.

Grossman, Gene M./Krueger, Alan B. (1995): Economic Growth and the Environment. In: Quarterly Journal of Economics Jg. 110/2, S. 353-377.

Häder, Michael (2015): Empirische Sozialforschung: Eine Einführung. Wiesbaden: Springer VS.

Harring, Niklas/Jagers, Sverker C./Martinsson, Johan (2011): Explaining Ups andDowns in the Public's Environmental Concern in Sweden. In: Organization \& Environment Jg. 24/4, S. 388-403. Hauff, Volker (1987): Unsere gemeinsame Zukunft: Der Brundtland-Bericht der Weltkommission für Umwelt und
Entwicklung. Greven: Eggenkamp.

Hauff, Volker/Scharpf, Fritz Wilhelm (1975): Modernisierung der Volkswirtschaft: Technologiepolitik als Strukturpolitik. Frankfurt am Main: Europäische Verlagsanstalt.

Henkel, Anna (2016): Natur, Wandel, Wissen: Beiträge der Soziologie zur Debatte um nachhaltige Entwicklung. In: Soziologie und Nachhaltigkeit Jg. 2/1, https://doi. org/10.17879/sun-2016-1675.

Hillebrandt, Frank (2013): Praxistheorie. In: Kneer, Georg/ Schroer, Markus (Hrsg.): Handbuch soziologische Theorien. Wiesbaden: Springer VS. S. 369-394.

Howes, Michael James/McKenzie, Marteena/Gleeson, Bredan/Gray, Rowan/Byrne, Jason Antony/Daniels, Peter (2010): Adapting ecological modernisation to the Australian context. In: Journal of Integrative Environmental Sciences Jg. 7/1, S. 5-21.

Huber, Joseph (1982): Die verlorene Unschuld der Ökologie: Neue Technologien und superindustrielle Entwicklung. Frankfurt am Main: S. Fischer.

Huber, Joseph (1995): Nachhaltige Entwicklung durch Suffizienz, Effizienz und Konsistenz. In: Fritz, Peter/Huber, Joseph/Levi, Hans Wolfgang (Hrsg.): Nachhaltigkeit in naturwissenschaftlicher und sozialwissenschaftlicher Perspektive. Stuttgart: Hirzel, S. 31-46.

Huber, Joseph (2011): Allgemeine Umweltsoziologie. Wiesbaden: Springer VS.

Jänicke, Martin (1993): Ökologische und politische Modernisierung in entwickelten Industriegesellschaften. In: Prittwitz, Volker von (Hrsg.): Umweltpolitik als Modernisierungsprozeß: Politikwissenschaftliche Umweltforschung und -lehre in der Bundesrepublik Deutschland. Wiesbaden: Springer VS, S. 15-29.

Jonas, Michael/Littig, Beate/Wroblewski, Angela (2017): Object, Perspectives and Methodology of Praxeological Research. In: dies. (Hrsg.): Methodological reflections on practice oriented theories. Cham, Schweiz: Springer, S. 251-261.

Kaiser, Henry F./Dickman, Kern (1959): Analytic determination of common factors. In: American Psychologist Jg. 14, S. 425-439.

Kallis, Giorgos/Demaria, Federico/D‘Alisa, Giacomo (2016): Degrowth. In: D'Alisa, Giacomo/Demaria, Federico/ 
Kallis, Giorgos (Hrsg.): Degrowth: Handbuch für eine neue Ära. München: oekom, S. 17-38.

Kangas, Jarkko (2019): Picturing two modernities: Ecological modernisation and the media imagery of climate change. In: Nordicom Review Jg. 40/1, S. 61-74.

Kössler, Georg (2013): Die Klimabewegung in Deutschland. In: Dietz, Matthias/Garrelts, Heiko (Hrsg.) Die internationale Klimabewegung: Ein Handbuch. Dordrecht: Springer VS, S. 185-204.

Kropp, Ariane (2019): Grundlagen der Nachhaltigen Entwicklung: Handlungsmöglichkeiten und Strategien zur Umsetzung. Wiesbaden: Springer Gabler.

Latouche, Serge (2009): Farewell to growth. Cambridge: Polity Press.

Li, Ding/ZhaDingo, Luman/Ma, Shuang/Shao, Shuai/ Zhang, Lixiao (2019): What influences an individual's pro-environmental behavior? A literature review. In: Resources, Conservation and Recycling Jg. 146, S. 28-34.

Lorenz, Stephan (2014): Mehr oder weniger?: Zur Soziologie ökologischer Wachstumskritik und nachhaltiger Entwicklung. Bielefeld: transcript.

Lorenz, Stephan (2016): Degrowth und Postwachstum Reflexionen zu Konzeptvielfalt und Nachhaltigkeitsbezügen. Zeitschrift für Technikfolgenabschätzung in Theorie und Praxis Jg. 25/2, S. 7-14.

Machin, Amanda (2019): Changing the story? The discourse of ecological modernisation in the European Union. In: Environmental Politics Jg. 28/2, S. 208-227.

Meadows, Dennis L./Meadows, Donella H./Zahn, Erich/ Milling, Peter/Heck, Hans-Dieter (1972): Die Grenzen des Wachstums: Bericht des Club of Rome zur Lage der Menschheit. Stuttgart: Deutsche Verlags-Anstalt.

Mol, Arthur P.J. (2002): Ecological Modernization and the Global Economy. In: Global Environmental Politics Jg. 2/2, S. 92-115.

Mol, Arthur P.J./Spaargaren, Gert (2000): Ecological modernisation theory in debate: A review. In: Environmental Politics Jg. 9/1, S. 17-49.

Mol, Arthur P.J./Spaargaren, Gert/Sonnenfeld, David A. (2014): Ecological modernization theory: where do we stand? In: Bemmann, Martin/Metzger, Birgit/Roderich, von Detten (Hrsg.): Ökologische Modernisierung: Zur Geschichte und Gegenwart eines Konzepts in Umweltpolitik und Sozialwissenschaften. Frankfurt am Main: Campus, S. 33-66.

Möltner, Andreas/Schellberg, Dieter/Jünger, Jana (2006): Grundlegende quantitative Analysen medizinischer Prüfungen. In: GMS Zeitschrift für Medizinische Ausbildung Jg. 23/ 3, S. 1-11.

Moosbrugger, Helfried/Schermelleh-Engel, Karin (2012): Exploratorische (EFA) und Konfirmatorische Faktorenanalyse (CFA). In: Moosbrugger, Helfried/Kelava, Augustin (Hrsg.): Testtheorie und Fragebogenkonstruktion. Berlin: Springer, S. 325-343.

Neugebauer, Birgit (2004): Die Erfassung von Umweltbewusstsein und Umweltverhalten. ZUMA-Methodenbericht Nr. 2004/07.

Organisation for Economic Co-operation and Development (OECD) (2011): Towards Green Growth. Paris: OECD Publishing.

Paech, Niko (2012): Vom grünen Feigenblatt zur Postwachstumsökonomie. In: Ökologisches Wirtschaften Jg. 27/4, S. 17-19.

Prittwitz, Volker von (1993): Vorwort. In: ders. (Hrsg.): Umweltpolitik als Modernisierungsprozeß: Politikwissenschaftliche Umweltforschung und -lehre in der Bundesrepublik Deutschland. Wiesbaden: Springer VS, S. 7-13.

Rockström, Johan/Steffen, Will/Noone, Kevin/Persson, Åsa/Chapin III, F. Stuart/Lambin, Eric/Lenton, Timothy M./Scheffer, Marten/ Folke, Carl/ Schellnhuber, Hans Joachim/ Nykvist, Björn/de Wit, Cynthia A/Hughes, Terry/van der Leeuw, Sander/Rodhe, Henning/Sörlin, Sverker/Snyder, Peter K/Costanza, Robert/Svedin, Uno/ Falkenmark, Malin/Karlberg, Louise/Corell, Robert W./ Fabry, Victoria J/Hansen, James/Walker, Brian/Liverman, Diana/Richardson, Katherine/Crutzen, Paul/Foley, Jonathan A (2009): A safe operating space for humanity. In: Nature Jg. 461/7263, S. 472-475.

Rödel, Michael (2013): Die Invasion der „Nachhaltigkeit“: Eine linguistische Analyse eines politischen und ökonomischen Modeworts. In: Deutsche Sprache Jg. 41/2, S. 115-141.

Scherhorn, Gerhard/Haas, Hendrik/Hellenthal, Frank/ Seibold, Sabine (2014): Gütergebundenheit: Zusammen- 
stellung sozialwissenschaftlicher Items und Skalen. Mannheim: ZIS - GESIS Leibniz Institute for the Social Sciences.

Schwegler, Regina/Spescha, Gina/Schäppi, Bettina/Iten, Rolf (2015): Klimaschutz und Grüne Wirtschaft - was meint die Bevölkerung?: Ergebnisse einer repräsentativen Bevölkerungsbefragung. Zürich.

Seidl, Irmi/Zahrnt, Angelika (2010): Die Postwachstumsgesellschaft: Wie der Abschied vom Paradigma des Wirtschaftswachstums gelingen kann. In: Ökologisches Wirtschaften Jg. 25/3, S. 18-19.

Shove, Elizabeth (2010): Beyond the ABC: Climate Change Policy and Theories of Social Change. In: Environment and Planning A: Economy and Space Jg. 42/6, S. 1273-1285.

Simonis, Udo E. (1984): Ökologische Modernisierung der Wirtschaftspolitik. In: Gewerkschaftliche Monatshefte Jg. 4, S. 197-214.

Solheim, Erik (2017): Foreword. In: Green Industrial Policy. Concept, Policies, Country Experiences. Geneva: UN Environment/Deutsches Institut für Entwicklungspolitik (DIE).

United Nations (2016): Paris Agreement. Certified true copy (XXVII-7-d). Paris.

United Nations Conference on Environment and Development (UNCED) (1992): Convention on Biological Diversity. Rio de Janeiro.

United Nations Economic and Social Council (2014): Emerging issues: the social drivers of sustainable development: E/CN.5/2014/8. Commission for Social Development/ Fifty-second session 11-21/ February 2014 Item 3 (c) of the provisional agenda ${ }^{*}$ / Follow-up to the World Summit for Social Development and the twenty-fourth special session of the General Assembly. Geneva.

United Nations Environment Programme (UNEP) (2011): Towards a Green Economy: Pathways to Sustainable Development and Poverty Eradication. A Synthesis for Policy Makers. St-Martin-Bellevue.

United Nations General Assembly (2015): Transforming our world: The 2030 Agenda for Sustainable Development. A/RES/70/1. [United Nations Division for Sustainable Development]. New York.

Victor, Peter A. (2016): Wachstum. In: D'Alisa, Giacomo/ Demaria, Federico/Kallis, Giorgos (Hrsg.): Degrowth:
Handbuch für eine neue Ära. München: oekom, S. 188-192.

Weiss, Martin/Cattaneo, Claudio (2017): Degrowth - Taking Stock and Reviewing an Emerging Academic Paradigm. In: Ecological economics: the journal of the International Society for Ecological Economics Jg. 137, S. 220-230.

Wingerter, Christian (2001): Allgemeines Umweltbewusstsein. Mannheim: ZIS - GESIS Leibniz Institute for the Social Sciences.

World Commission on the Environment and Development (WCED) (1987): A/42/427 - Report of the World Commission on Environment and Development: Note by the Secretary General. Our Common Future.

York, Richard/Rosa, Eugene A./Dietz, Thomas (2003): Footprints on the Earth: The Environmental Consequences of Modernity. In: American Sociological Review Jg. 68/2, S. 279-300.

\section{ZUM AUTOR}

Jonathan Schreiber, 25, studierte in Hamburg Soziologie und Medien- und Kommunikationswissenschaft. Gegenwärtig studiert er Global Sustainability Science in Lüneburg. Seine Forschungsinteressen umfassen die Gebiete Umweltsoziologie, Nachhaltigkeitskommunikation sowie Transitions- und Innovationsforschung.

\section{Der Beitrag wurde von Daniel Bräunling, Markus Kohlmeier, Nils Haacke, Andreas Schulz und Hendrik Erz gereviewt, redak- tionell betreut und lektoriert.}


ANHANG

\begin{tabular}{|c|c|c|}
\hline Item ID & Operationalisierung/Item & Theoretische Fundierung \\
\hline O1 & $\begin{array}{l}\text { Die moderne Industriegesellschaft hat ihr Ende } \\
\text { erreicht* }\end{array}$ & $\begin{array}{l}\rightarrow \text { Ziel der konsistenz- und effizienzorien- } \\
\text { tierten Erneuerung der Industrie. } \\
\rightarrow \text { „Postulate, die moderne Industrie- } \\
\text { gesellschaft habe ihr historisches Ende er- } \\
\text { reicht, werden [...] zurückgewiesen“ (Huber } \\
\text { 2011: 143). }\end{array}$ \\
\hline $\mathrm{O} 2$ & $\begin{array}{l}\text { Unsere globalen Umweltprobleme lassen sich } \\
\text { durch die Entwicklung und den Einsatz neuer } \\
\text { Technologien lösen }\end{array}$ & $\begin{array}{l}\rightarrow \text { Innovationen und Modernisierung } \\
\text { als entscheidende Faktoren nachhaltiger } \\
\text { Entwicklung (vgl. Fisher/Freudenburg 2001: } \\
702 \text { ). } \\
\rightarrow \text { Annahme, gegenwärtige Nachhaltig- } \\
\text { keitsprobleme ließen sich durch techno- } \\
\text { logischen Fortschritt lösen (vgl. Drews et al. } \\
\text { 2019: 143). }\end{array}$ \\
\hline O3 & $\begin{array}{l}\text { Innovative Unternehmen in Deutschland soll- } \\
\text { ten mehr mit öffentlichen Geldern unterstützt } \\
\text { werden }\end{array}$ & \multirow{2}{*}{$\begin{array}{l}\rightarrow \text { Etablierung der notwendigen Inno- } \\
\text { vationen ,durch geeignete ökonomische } \\
\text { und rechtlich-administrative Rahmen- } \\
\text { bedingungen“ (Huber 2011: 143). }\end{array}$} \\
\hline $\mathrm{O} 4$ & $\begin{array}{l}\text { Neue Gesetze sollten für ein besseres Innova- } \\
\text { tionsklima in Deutschland sorgen }\end{array}$ & \\
\hline O5 & $\begin{array}{l}\text { "Wissenschaft und Technik werden die globalen } \\
\text { Umweltprobleme lösen, ohne dass wir unsere } \\
\text { Lebensweise grundlegend ändern müssen" } \\
\text { (Schwegler et al. 2015: 10) }\end{array}$ & $\begin{array}{l}\rightarrow \quad \text { Grundlegende Umstrukturierung der } \\
\text { Gesellschaft ist nicht notwendig (vgl. York et } \\
\text { al. 2003: 285). }\end{array}$ \\
\hline O6 & $\begin{array}{l}\text { Ein konsequenter Umweltschutz würde sich } \\
\text { positiv auf die Wettbewerbsfähigkeit der Wirt- } \\
\text { schaft auswirken (vgl. BMUB/UBA 2017: 20) }\end{array}$ & $\begin{array}{l}\rightarrow \text { Umweltschutz und Wirtschaft sind } \\
\text { keine unvereinbaren Gegensätze (vgl. Huber } \\
\text { 2011: 148). } \\
\rightarrow \text { Umweltschutz kann Wirtschaftswachs- } \\
\text { tum unterstützen (vgl. Kangas 2019: 63). }\end{array}$ \\
\hline O7 & $\begin{array}{l}\text { „Um die Umwelt schützen zu können, braucht } \\
\text { Deutschland wirtschaftliches Wachstum" } \\
\text { (Wingerter 2001: 2) }\end{array}$ & $\begin{array}{l}\rightarrow \quad \text { Umweltschutz führt nicht nur zu mehr } \\
\text { wirtschaftlichem Wachstum, sondern wird } \\
\text { erst durch dieses möglich (vgl. Grossman/ } \\
\text { Krueger 1995: 370f.). }\end{array}$ \\
\hline O8 & $\begin{array}{l}\text { Der Nutzen neuer Technologien ist größer als } \\
\text { die möglicherweise auftretenden schädlichen } \\
\text { Auswirkungen (vgl. Belitz/Kirn 2008: 58) }\end{array}$ & $\begin{array}{l}\rightarrow \text { Optimistisches Vertrauen in Technik } \\
\text { (vgl. Mol/Spaargaren 2000: 20). }\end{array}$ \\
\hline O9 & $\begin{array}{l}\text { Es ist nichts dabei, ein neues, verbessertes Ge- } \\
\text { rät zu kaufen, auch wenn das alte Modell noch } \\
\text { funktioniert (vgl. Scherhorn et al. 2014: 2) }\end{array}$ & $\begin{array}{l}\rightarrow \text { Obwohl „Basisinnovationen“ gegenüber } \\
\text { "nachfolgenden Innovationen“ größere } \\
\text { Wichtigkeit zukommt, sind auch letztere von } \\
\text { Bedeutung (Huber 2011: 172). }\end{array}$ \\
\hline \multicolumn{3}{|c|}{$\begin{array}{l}\text { Anmerkungen: Negativ gepolte Items sind mit * versehen. Items, welche im Wortlaut aus bestehenden } \\
\text { Studien entnommen wurden, werden mit " und entsprechender Quelle gekennzeichnet. Aus bestehenden } \\
\text { Studien paraphrasierte Items werden mit (vgl.) gekennzeichnet. }\end{array}$} \\
\hline
\end{tabular}

Tabelle 2: Index für ökologische Modernisierung 


\begin{tabular}{|c|c|c|}
\hline Item ID & Operationalisierung/Item & Theoretische Fundierung \\
\hline P1 & $\begin{array}{l}\text { "Es gibt natürliche Grenzen des Wachstums, } \\
\text { die unsere industrialisierte Welt längst erreicht } \\
\text { oder schon überschritten hat" (BMUB/UBA } \\
\text { 2017: 20) }\end{array}$ & $\begin{array}{l}\rightarrow \text { Die Menschheit lebt über ihre } \\
\text { Verhältnisse (vgl. Latouche 2009: 7f.). }\end{array}$ \\
\hline $\mathrm{P} 2$ & $\begin{array}{l}\text { "Wir müssen Wege finden, wie wir unabhängig } \\
\text { vom Wirtschaftswachstum gut leben können" } \\
\text { (BMUB/UBA 2017: 20) }\end{array}$ & $\begin{array}{l}\rightarrow \text { Ziel einer Neuanordnung, ein } \\
\text { „Restructure“ (Latouche 2009: 36). }\end{array}$ \\
\hline $\mathrm{P} 3$ & $\begin{array}{l}\text { „Das globale Wirtschaftswachstum führt } \\
\text { zwangsläufig zu immer mehr Umweltzerstö- } \\
\text { rung und Klimawandel“ (Schwegler et al. } \\
\text { 2015: 10) }\end{array}$ & $\begin{array}{l}\rightarrow \text { „Wachstum ohne Naturzerstörung ist eine } \\
\text { Illusion“ (Eversberg/Schmelzer 2015: 7). } \\
\rightarrow \text { Konsensuales Item der Degrowth- } \\
\text { Bewegung, hier durch ein gemeinverständ- } \\
\text { licheres Statement von Schwegler et al. } \\
\text { (2015) operationalisiert. }\end{array}$ \\
\hline $\mathrm{P} 4$ & $\begin{array}{l}\text { „Wir werden in Zukunft auf Annehmlichkeiten } \\
\text { verzichten müssen, an die wir uns gewöhnt } \\
\text { haben“(Eversberg/Schmelzer 2015: 7) }\end{array}$ & $\begin{array}{l}\rightarrow \text { Ziel ist es, weniger zu produzieren und } \\
\text { zu konsumieren, gefasst wird dies unter dem } \\
\text { Schlagwort „Reduce“ (Latouche 2009: 38). } \\
\rightarrow \text { Konsensuales Item der Degrowth- } \\
\text { Bewegung (vgl. Eversberg/Schmelzer 2015: 7). }\end{array}$ \\
\hline P5 & $\begin{array}{l}\text { „Es hat keinen Sinn, den Kapitalismus ab- } \\
\text { zulehnen, solange es keinen umsetzbaren gesell- } \\
\text { schaftlichen Gegenentwurf gibt }{ }^{\star *} \text { (Eversberg/ } \\
\text { Schmelzer 2015: 7)* }\end{array}$ & $\begin{array}{l}\rightarrow \quad \text { Mit „Reconceptualize“ soll ein neues } \\
\text { Werteverständnis erreicht werden (Latouche } \\
\text { 2009: 35). } \\
\rightarrow \quad \text { Konsensuales Item der Degrowth-Bewe- } \\
\text { gung (vgl. Eversberg/Schmelzer 2015: 7). }\end{array}$ \\
\hline P6 & $\begin{array}{l}\text { "Jede und jeder ist für die schädlichen Folgen } \\
\text { des eigenen Lebensstils selbst verantwortlich" } \\
\text { (Eversberg/Schmelzer 2015: 8) }\end{array}$ & $\begin{array}{l}\rightarrow \quad \text { Unter dem Stichwort „Re-evaluate“ } \\
\text { sollen neue Werte etabliert werden (Latouche } \\
\text { 2009: 34). }\end{array}$ \\
\hline P7 & $\begin{array}{l}\text { "Die globalen Umweltprobleme können nur } \\
\text { bewältigt werden, wenn wir alle weniger kon- } \\
\text { sumieren" (Schwegler et al. 2015: 10) }\end{array}$ & $\begin{array}{l}\rightarrow \quad \text { Mit "Reduce" wird die Reduktion von } \\
\text { Konsum und Abfall gefordert (Latouche } \\
\text { 2009: 38; Ančić/Domazet 2015: 457). }\end{array}$ \\
\hline P8 & $\begin{array}{l}\text { "Angesichts der begrenzten natürlichen } \\
\text { Ressourcen müssen die reichen Länder ihr } \\
\text { Wirtschaftswachstum einschränken, um sicher- } \\
\text { zustellen, dass arme Länder einen angemesse- } \\
\text { nen Lebensstandard erreichen können" (Drews } \\
\text { et al. 2019: 143) }\end{array}$ & $\begin{array}{l}\rightarrow \quad \text { Unter dem Stichwort "Redistribute" } \\
\text { werden Gerechtigkeitsfragen aufgegriffen } \\
\text { (Latouche 2009: 36). }\end{array}$ \\
\hline P9 & $\begin{array}{l}\text { Die Zukunft unserer Nahrungsmittelversor- } \\
\text { gung liegt in der lokalen, kleinbäuerlichen } \\
\text { Landwirtschaft. }\end{array}$ & $\begin{array}{l}\rightarrow \text { Mit „Relocalize“ wird eine Relokalisie- } \\
\text { rung gefordert (Latouche 2009: 37). }\end{array}$ \\
\hline
\end{tabular}

Tabelle 3: Index für Postwachstum 


\section{Von Aktionen und Methoden}

Letzte Chance für einen grünen Aufschwung: Mit dem Green New Deal zu einer klimaneutralen Wirtschaft

»Rational und brutal ehrlich.« taz

»Eine eloquente Anklage gegen Ausreden und Verzögerungstaktiken und der Aufruf, endlich zu tun, was getan werden kann.« Michael Soder, Falter

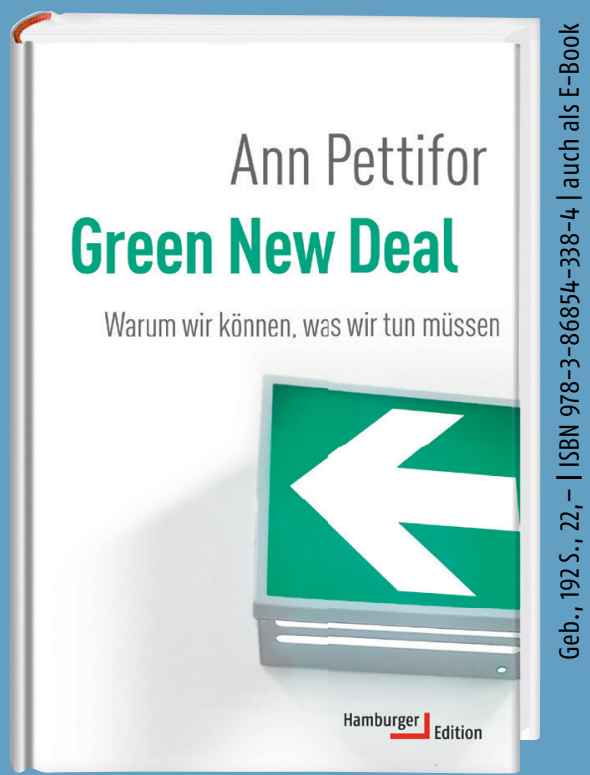

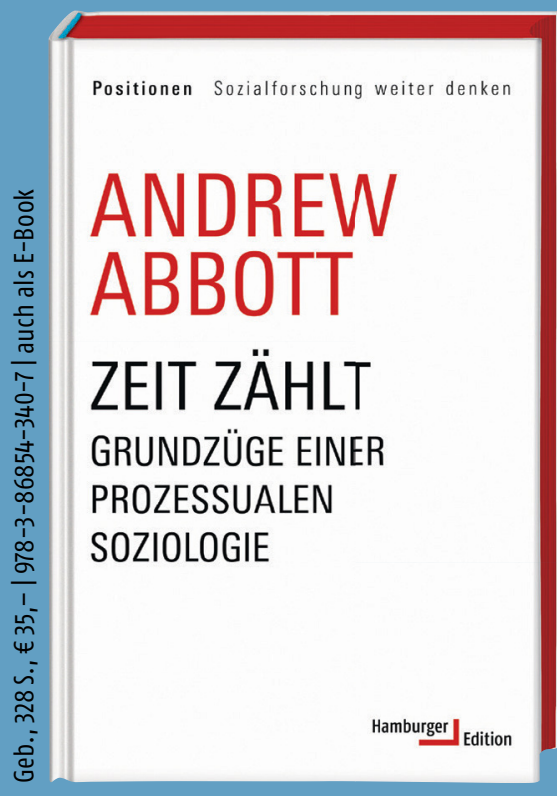

Andrew Abbott plädiert für eine prozessual orientierte Sozialforschung, die die Temporalität des Sozialen in der soziologischen Theoriebildung verankert. Nicht die Stabilität gesellschaftlicher Verhältnisse ist der Normalfall, sondern ihr Wandel.

Mit diesem Band liegen erstmals ausgewählte Aufsätze von Andrew Abbott vor. Sie eröffnen den Zugang zu einem Autor, der in den USA und Frankreich längst zu den prominentesten Sozialwissenschaftlern unserer Zeit gehört. Mit einer Einführung von Thomas Hoebel, Wolfgang Knöbl und Aaron Sahr

\section{Hamburger}




\title{
Bäume, die die Welt
}

\section{bedeuten}

\author{
Der Hambacher Forst als Symbol der \\ deutschen Klimabewegung
}

von Ruben Kaiser

\begin{abstract}
Welche Transformationen durchläuft ein in der Klimaforschung gewonnenes Wissen, um in ein gesellschaftliches Problem politischer Tragweite überführt zu werden? Um besser zu verstehen, wie ein neues Umweltbewusstsein sozial hergestellt wird, lohnt es sich die Arbeit jener sozialen Bewegungen zu untersuchen, die mit ihrer Praxis Einfluss auf die Deutung der Klimakrise ausüben. Der vorliegende Artikel widmet sich daher Formierungsprozessen von Klimaprotesten am Beispiel der Auseinandersetzung um die Zukunft des Hambacher Forsts. Dabei wird argumentiert, dass es weniger die am Protest beteiligten Organisationen sind, die für den Bewegungsaufbau eine zentrale Rolle spielen, sondern niedrigschwellige integrative Versammlungsformate. Am konkreten Beispiel des Hambacher Forstes wird auf die Bedeutung der monatlichen Waldspaziergänge eingegangen. Diese sind in der Lage, Kommunikationsbarrieren abzubauen und durch den direkten Kontakt zwischen verschiedenen Akteur*innen ein Gemeinschaftsgefühl entstehen zu lassen.
\end{abstract}

\section{Schlagwörter}

Klimabewegung; Hambacher Forst; Übersetzung; Akteur-Netzwerk Theorie 


\section{Einleitung}

In den letzten Jahren hat der Themenkomplex Umwelt- und Klimaschutz stetig an politischer Bedeutung hinzugewonnen. So gaben etwa anlässlich der Europawahl im Mai 201948 Prozent der Befragten an, das Thema spiele für ihre Wahlentscheidung eine große Rolle (vgl. Infratest dimap 2019). Seit Januar 2019 machen Schüler*innen durch das Bestreiken des Unterrichts auf eine aus ihrer Sicht verfehlte Klimapolitik der Bundesregierung aufmerksam und Aktionsbündnisse wie Extinction Rebellion oder Ende Gelände generieren mit öffentlichkeitswirksamen Blockadeaktionen Aufmerksamkeit und unterstreichen damit die Dringlichkeit der Lage. Gleichzeitig verfügt die Klimaforschung heute - 32 Jahre nach der Gründung des Intergovernmental panel on climate change (IPCC) - über ein umfangreiches Reservoir an gefestigtem Wissen über Ursachen und Risiken der globalen Erderwärmung. Auch Klimaaktivist*innen werden nicht müde zu betonen, „die Politik“ müsse lediglich dem folgen, was ,die Wissenschaft“ seit längerer Zeit fordere (vgl. Thunberg 2019).

Mit den Worten Ulrich Becks lässt sich der Klimawandel als eine neuartige Gefährdungslage globalen Ausmaßes verstehen, die kennzeichnend für den Übergang von der modernen Industriegesellschaft zur sogenannten Risikogesellschaft ist. Das Be- sondere an ebenjenen Gefährdungslagen ist, dass in ihnen Lebenslagen und Wissensproduktion "miteinander verschränkt" sind (Beck 2016: 72). Da sich abstrakte Risiken wie der Klimawandel nur schwer sinnlich erfahren lassen, kommt der Produktion und Vermittlung von Wissen eine entscheidende Rolle zu. Wenn aber ausreichend Wissen über das Problem einer Erhöhung der globalen Durchschnittstemperatur vorhanden ist und in der Klimaforschung weitestgehend Konsens darüber besteht, dass diese vom Menschen verursacht wurde, dann stellt sich die Frage: Wieso wird dieses Thema so kontrovers diskutiert und warum reagieren die politisch Verantwortlichen nur so zaghaft auf die Forderungen der Klimabewegung?

\section{Soziale Bewegungen als Mittler}

Eine auf diese Weise gestellte Frage lässt jedoch zu viele Zwischenschritte aus, um einer angemessenen Auseinandersetzung mit dem Thema gerecht zu werden. Sie impliziert, ein naturwissenschaftlicher Konsens auf dem Gebiet der Klimaforschung müsse automatisch einen gesellschaftlichen Konsens über eine ambitionierte Klimapolitik (zum Beispiel ein schneller Ausstieg aus fossilen Energien oder eine höhere Besteuerung des Flugverkehrs) zur Folge haben. Erkenntnisse der Klimaforschung und konkrete politische Maßnahmen zur Eindämmung der Erderwärmung werden 
auf diese Weise als zwei Seiten derselben Medaille angesehen.

Dabei wird außer Acht gelassen, dass aus der Klimaforschung gewonnene wissenschaftliche Erkenntnisse ,übersetzt“ (Ich verwende den Begriff der Übersetzung in Anlehnung an Michel Callons und Bruno Latours „,Soziologie der Übersetzung "; Latour 2014; Callon 1986) werden müssen, damit sie etwa für politisches Handeln verwertbar sind. Mit anderen Worten: Es bedarf Arbeit, um ein naturwissenschaftliches Problem in ein gesellschaftliches Problem beziehungsweise in ein politisches Programm zu überführen. Die Soziologie kann jene Arbeit aufzeigen, mittels derer ein neues Umweltbewusstsein beziehungsweise ein klimapolitischer Handlungsdruck hergestellt wird, indem zuvor nicht-assoziierte Entitäten miteinander in Verbindung gebracht werden. Doch an welcher Stelle sollte eine Analyse der Übersetzungsarbeit ansetzen?

Um die Erkenntnis des menschengemachten Klimawandels in ein politisches Programm zu übersetzen, bedarf es unter anderem zivilgesellschaftliche Akteur ${ }^{*}$ innen und soziale Bewegungen, die ihrer Version von einer ambitionierten Klimapolitik im öffentlichen Diskurs Ausdruck verleihen können. Daher bietet es sich an, jene soziale Bewegungen konkreter unter die Lupe zu nehmen, die in den letzten Jahren wesentlich dazu beigetragen haben, dass das Thema Klimakrise in den Mittelpunkt der politischen Auseinandersetzung gerückt ist. Eine soziologische Analyse der "gesellschaftlichen Naturbeziehungen“ (Brand 2014: 7) muss sich mit Protest- und Bewegungsforschung auseinandersetzen.

In den vorherigen Abschnitten wurde auf die Relevanz von Übersetzungsarbeit für die erfolgreiche Transformation von Wissen in politische Handlungsprogramme eingegangen. Auf der Makroebene vollziehen sich diese Prozesse über Ketten von Mittlern. In Abgrenzung zu einem „Zwischenglied“, ist ein solcher Mittler nach Bruno Latour immer imstande, die Bedeutung jener Elemente zu transformieren, die er verhandelt. Er ist kein bloßes Transportmedium dessen Output bereits durch den Input vorgegeben ist (vgl. Latour 2014: 70). In Bezug auf die verschiedenen Deutungen zur Bekämpfung der Klimakrise können Umwelt-NGOs und soziale Bewegungen als ein Mittler unter vielen angesehen werden, die das Wissen über die globale Erderwärmung auf ihre Weise transformieren und weiterleiten. Die Soziologie der Übersetzung lehrt uns, den Blick auf die vielen Zwischenstationen zu lenken, die vonnöten sind, um komplexe Verbindungen zwischen heterogenen Entitäten aufzubauen und zu verstetigen. Vor diesem Hintergrund erscheint es wenig sinnvoll, von sozialen Bewegungen als Einheit mit festen Grenzen auszugehen. Stattdessen wird in diesem Artikel eine Ebene weiter unten angesetzt werden. 
Sein zentrales Anliegen ist, aufzuzeigen, dass Übersetzungsarbeit ebenso für das bessere Verständnis der Konstitution und Stabilisierung sozialer Bewegungen auf der Mikroebene von Bedeutung ist. Denn auch das Versammeln unterschiedlicher Akteur*innen hinter einem gemeinsamen Projekt sowie die Stabilisierung des Netzwerks nach außen ist auf Übersetzungsarbeit von unterschiedlichen Mittlern angewiesen.

\section{Baumhäuser gegen Braunkohle}

Exemplarisch soll dies am Forschungsgegenstand Hambacher Forst illustriert werden, genauer gesagt anhand der Proteste gegen dessen geplante Rodung durch den Kohlekonzern RWE. Dabei stütze ich meine Argumentation auf die Ergebnisse einer ethnographischen Feldstudie, die ich im Rahmen meiner Abschlussarbeit an der Universität Paris-Nanterre konzipiert und durchgeführt habe. Während eines einmonatigen Aufenthalts im rheinischen Braunkohlerevier wurden 19 Interviews mit verschiedenen Beteiligten der Widerstandsbewegung sowie mit Vertreter*innen der Gegenseite (RWE, Bergbaugewerkschaft IG BCE) geführt und Veranstaltungen und Gruppensitzungen mittels eines Feldnotizbuchs dokumentiert. Dabei orientierte sich der Interviewleitfaden an folgender Problemstellung: Wie war es möglich, eine ganze Palette an unterschiedlichen und auf den ersten Blick inkompatibel erscheinenden Akteur ${ }^{*}$ innen gemeinsam gegen die Landesregierung und RWE zu mobilisieren?

Anstatt das Hauptaugenmerk der Analyse auf konjunkturelle Faktoren und die Frage eines günstigen gesellschaftlichen Kontexts zu lenken (Der Fachbegriff in der Bewegungssoziologie ist die Gelegenheitsstruktur, vgl. McAdam 1982; Tarrow 2005), möchte ich mich in diesem Artikel mit der Frage des Aufbaus und der Stabilisierung jener Netzwerke beschäftigen, mithilfe derer eine derart breite Mobilisierung im Herbst 2018 erst möglich wurde. Denn die verschiedenen Organisationen und Gruppierungen (Umwelt-NGOs, Bürger*inneninitiativen, informelle Aktionsbündnisse, anarchistische Waldbesetzung) blicken auf eine gewisse Kontinuität des Protests (erstes Klimacamp im Rheinland 2010, Erste Waldbesetzung 2012, Erste EndeGelände-Aktion 2015, mehrere größere Rote-Linie-Aktionen am Tagebaurand ab 2016) zurück und lassen sich demnach gemäß der Definition von Dieter Rucht und Roland Roth als soziale Bewegung charakterisieren (vgl. 2008: 13).

Um die innere Vielfalt dieser „sozialen Bewegung Hambacher Forst" soziologisch greifbar zu machen, wählte ich im Vorfeld des Feldaufenthalts einen von Lilian Mathieu inspirierten Feldansatz, mittels dessen sich die verschiedenen am Protest beteiligten Gruppen anhand ihrer 
Struktur und inhaltlichen Ausrichtung, verschiedenen „Polen“ innerhalb des „Bewegungsraums“ zuordnen ließen (für weiterführende Informationen zum Konzept des „espace des mouvements sociaux“ siehe: Mathieu 2012). Auf diese Weise entstand die Arbeitshypothese, die eher bürgerlichen Akteur*innen hätten sich im Vorfeld der Räumung den eher radikalen Kräften inhaltlich angenähert und daher enger untereinander kooperieren können. Allerdings widersprach diese Deutung im Wesentlichen den Erfahrungen im Feld. Letztere führten folglich zu dem Schluss, dass eine zu starke Fokussierung auf den Beitrag der einzelnen Organisationen nicht ausreichend ist, um die kollektive verbindende Dimension der Bewegung zu erklären. Stattdessen stellte es sich als fruchtbar heraus, die Aufmerksamkeit auf jene Formate zu lenken, die imstande waren, die gesamte Palette an Akteur*innen kollektiv $\mathrm{zu}$ versammeln und so wesentlich zur Herausbildung einer niedrigschwelligen Bewegungsidentität beitrugen.

Ein derartige konzeptuelle Ausrichtung sieht soziale Bewegungen weniger als prädiskursiv existierende Entitäten an, die von rational handelnden Akteur ${ }^{*}$ innen oder strukturellen Zwängen geprägt sind (wie es z.B. der Framing-Ansatz, die Ressource Mobilization oder die in Frankreich populären Feldansätze postulieren, vgl. Benford/Snow 2000; McCarthy/Zald 1977). Stattdessen interessiert sich ein auf diese Weise ausgerichteter Ansatz im Sinne einer „poststrukturalistischen Blickverschiebung" (Hagemann et al. 2019: 21ff.) für jene Prozesse, mittels derer sich soziale Bewegungen erst konstituieren. Übersetzungsarbeit ist einer dieser Prozesse, welcher im Rahmen dieses Artikels exemplarisch am Beispiel der monatlichen Waldspaziergänge im Hambacher Wald analysiert werden soll.

In einem ersten Abschnitt wird daher noch einmal detaillierter auf die Erfahrungen aus einem Monat Feldaufenthalt eingegangen. Es wird dargelegt, wieso eine Fokussierung auf die am Protest beteiligten Organisationen und deren Modellierung anhand eines Feldansatzes keine überzeugende Erklärung für den Zusammenhalt der Bewegung Hambacher Forst im Herbst 2018 liefern konnte. In einem zweiten Abschnitt wird dann mit den monatlichen Waldspaziergängen ein für die Bewegung bedeutendes Versammlungsformat herausgegriffen, mithilfe dessen die Stabilisierung des Netzwerks mittels Übersetzung besser erklärt werden kann. Die in diesem Artikel getroffenen Aussagen sind Ergebnis einer intensiven Auseinandersetzung mit dem Gegenstand Hambacher Forst und sozialen Bewegungen, erheben jedoch keinen Anspruch auf allgemeine Gültigkeit. Stattdessen sind die Ergebnisse als situiertes Wissen (vgl. Hale 2008: 13) und damit explizit auch als Anstoß für Diskussionen zu dem Thema zu verstehen. 


\section{Der Hambacher Forst, eine oder mehrere Bewegungen?}

Mit dem „größten Polizeieinsatz der Landesgeschichte" (Eyermann 31.12.2018) hatte die Regierung von Nordrhein-Westfalen ab Mitte September versucht, den seit 2012 besetzten Wald mitsamt den dort lebenden Aktivist ${ }^{\star}$ innen zu räumen. Da der Termin der Räumung jedoch unmittelbar vor die von RWE angesetzte tagebaubedingte Rodung des Waldes fiel, entwickelte sich der Hambacher Forst zum stands. Über mehrere Wochen hinweg mobilisierten NGOs und Aktivist ${ }^{\star}$ innennetzwerke ins rheinische Revier. Bilder von Sitzblockaden und Aktivist*innen, die von Baumhäusern geräumt wurden, fanden eine massenmediale Verbreitung und wurden lebhaft diskutiert. Am 5. Oktober verhängte des Oberverwaltungsgerichts Münster schließlich einen temporären Rodungsstopp für den Wald und am Folgetag wurde der Teilerfolg von 50.000 Menschen in und um den Wald herum gefeiert (vgl. Parth 8.10.2018).

Vor meinem Feldaufenthalt im rheinischen Revier hatte ich mithilfe einer Presserecherche und der bereits vorhandenen wissenschaftlichen Literatur (Bosse 2015; Sander 2016; Brock/Dunlap 2018; Kaufer/ Lein 2018) eine Liste jener Gruppen erstellt, die auf verschiedene Art und Weise an den Protesten gegen die Räumung

beteiligt waren (BUND NRW, Greenpeace, Buirer für Buir, Alle Dörfer bleiben, Aktion Unterholz, Ende Gelände, Waldbesetzung). Diese Gruppierungen ordnete ich daraufhin drei verschiedenen Organisationstypen $\mathrm{zu}$ : formelle Organisationen (BUND NRW, Greenpeace, Buirer für Buir, Alle Dörfer bleiben), informelle Aktivist ${ }^{\star}$ innenkollektive (Aktion Unterholz, Ende Gelände) und Gruppen ohne kollektive Identität (Waldbesetzung). Während erstere in der Regel als eingetragener Verein geführt werden und damit dem Vereinsrecht unterworfen sind, sehen sich Akteur*innen der übrigen zwei Kategorien eher als „Zusammenschluss freier Menschen", bei denen jeder ausschließlich für sich spreche (Hambacher Forst Buchprojekt 2015: 7; hambacherforst.org/besetzung/selbstverstaendnis/, 01.06.2020).

Die zentrale Herausforderung der ethnographischen Studie bestand darin, ein besseres Verständnis davon zu gewinnen, wie sich diese sehr unterschiedlichen Gruppierungen im Herbst 2018 hinter einem gemeinsamen Projekt vereinen und bis zu einem gewissen Grad eine Identität als Bewegung aufbauen konnten. Denn insbesondere im Hinblick auf die für sie als legitim geltenden Protestformen gab es deutliche Unterschiede. So ist es wenig überraschend, dass institutionalisierte Gruppen (BUND, Greenpeace, Buirer für Buir) sich eher als Impulsgeber für eine neue Politik innerhalb des bestehenden Systems definieren und ihr Aktionsrepertoire auf legale Formen 
der politischen Agitation beschränkt bleibt (zum Beispiel angemeldete Versammlungen oder öffentlichkeitswirksame FotoAktionen). Auf der anderen Seite sehen sich eher der öko-anarchistischen Szene entstammende Gruppierungen als radikale Opposition zum bestehenden politischökonomischen System und pflegen im Falle der Waldbesetzung eine radikale Offenheit gegenüber allen Aktionsformen (Im Gegensatz zu Ende Gelände und der Aktion Unterholz, die über einen Aktionskonsens verfügen, stellt sich die Waldbesetzung nicht prinzipiell gegen Sabotage und physische Gewalt; vgl. Aktion Unterholz 2018: 43). Und trotzdem: Im Vorfeld der Räumungen schlossen sich Vertreterinnen beider Lager zu Bündnissen zusammen und mobilisierten gemeinsam ins rheinische Revier. Wie war dieser hohe Grad an Kooperation möglich geworden, obwohl es doch gerade im Fall Hambacher Forst eine offensichtliche Trennung der Bewegung in ein radikales und ein bürgerliches Lager gegeben hatte? Diese Kategorien habe nicht ich als Soziologe ans Feld herangetragen, sondern sie werden von den Akteur*innen selbst verwendet. Auf der Waldbesetzung hat sich mit dem Wort „Bürgi“ ein eigener Begriff für die bürgerlichen Umweltschützer*innen etabliert. Das Attribut „radikal“ wird zum Beispiel von Ende Gelände selbst verwendet und als „Probleme an der Wurzel anpacken“ positiv umgedeutet (vgl. Interview Ende Gelände 2019: 312).

\section{Konfrontation mit Erfahrungen im Feld}

$\mathrm{Zu}$ Beginn des Feldaufenthalts war es meine Hypothese gewesen, dass sich bürgerliche und radikale Gruppierungen im Vorfeld der Räumungen inhaltlich angenähert hatten und es auf diese Weise für sie leichter wurde, in Netzwerken zu kooperieren. Doch diese Deutung der Ereignisse wurde durch die Erfahrungen vor Ort nicht bestätigt. Ganz im Gegenteil: vielmehr hatte ein Prozess der Entfremdung zwischen Teilen der Waldbesetzer*innen und bürgerlichen Umweltschutzorganisationen stattgefunden. So sprach ein ${ }^{\star} e$ Interviewpartner*in der „Buirer für Buir“ von einer geringeren Verlässlichkeit von Teilen der Waldbesetzung im Vergleich zu früheren Tagen.

Wenn wir aber gesagt haben: „Wir haben Gesprächsbedarf, wir müssen über das oder das sprechen.", dann war immer klar, dass in nem Plenum [...] ne Entscheidung getroffen wurde und auch diese Entscheidung äh für alle [...] ne Verbindlichkeit hatte. Und das ist heute nicht mehr.

(Interview Buirer für Buir 2019: 375ff.)

Wurden die Waldbesetzung und der BUND in der öffentlichen Wahrnehmung am engsten mit dem Kampf um den Erhalt des Waldes verbunden, so hatten ausgerechnet diese beiden Gruppen untereinander kaum 
Berührungspunkte und zum Teil auch widerstrebende Interessen. So sagte ein Interviewpartner vom BUND NRW:

\section{Mir macht momentan am meisten} Sorge, dass da die Baumbesetzer in bisher ungeschädigte Waldbereiche reingehen und da Rodungen dann provozieren, [...].

(Interview BUND NRW 2019: 419ff.)

Die vor Ort gewonnenen Daten standen also im Widerspruch zur Eingangshypothese. Protest beteiligten Netzwerke und Organisationen eine gemeinsame kollektive Identität teilten, denn von öko-anarchistischen bis hin zu bürgerlich-ökologischen Positionen waren sehr unterschiedliche Vorstellungen vorhanden. Die gewonnenen Eindrücke ließen daher den Schluss zu, dass es nicht sinnvoll war, die kollektive, verbindende Dimension der Proteste anhand der an ihm beteiligten Gruppen zu analysieren. Vielmehr stellte sich heraus, dass die „Bewegung Hambacher Forst" von der Vielzahl an Versammlungsformaten lebte, die durch ihre Niedrigschwelligkeit imstande waren, die gesamte Palette an Akteur*innen gemeinsam zu versammeln und trotz ihrer Unterschiedlichkeit miteinander ins Gespräch zu bringen.

Dies ist keine prinzipiell neue Erkenntnis, denn auch in der Vergangenheit haben soziale Bewegungen von regelmäßigen

Happenings an symbolträchtigen Orten profitiert, an denen die Bewegung erlebbar wurde. Das Paradebeispiel wäre im Fall der Anti-Atomkraft-Bewegung die jährlichen Aktionen gegen die Castortransporte ins Wendland. Für den konkreten Fall Hambach und die Klimabewegung möchte ich im Rahmen des vorliegenden Artikels auf die Bedeutung der Waldspaziergänge eingehen, die seit Mai 2014 einmal im Monat vom Naturführer Michael Zobel, seiner Partnerin Eva Töller sowie einer Gruppe von Freunden aus Aachen und Umgebung organisiert werden. Folgende These möchte ich im Hinblick auf die Bedeutung dieser Spaziergänge vertreten: Die Mobilisierung gegen die Räumung der Besetzung und für den Erhalt des Waldes konnte nur deshalb auf so breiter Basis stattfinden, weil es mit den Waldspaziergängen seit Jahren ein regelmäßiges Versammlungsformat gibt, das durch seine Niedrigschwelligkeit allen Akteurinnen gleichermaßen offen ist, zum gegenseitigen Austausch animiert und auf diese Weise einen wichtigen Anteil an der Herausbildung eines gemeinsamen Minimalkonsenses hat.

\section{Der Hambacher Wald, ein Ort mit hoher Symbolkraft}

Es ist nicht gleichbedeutend, an welchen Orten politische Konflikte ausgetragen werden und gerade in Bezug auf den so abstrakten Themenkomplex Klimawandel 


\section{Es ist nicht gleichbedeutend, an welchen Orten politische Konflikte ausgetragen werden und gerade in Bezug auf den so abstrakten Themenkomplex Klimawandel ist es von hoher Bedeutung inwieweit man imstande ist, symbolische Bilder zu generieren.}

ist es von hoher Bedeutung inwieweit man imstande ist, symbolische Bilder zu generieren. $\mathrm{Zu}$ nennen wären beispielsweise der Eisbär, dessen Lebensraum verschwindet oder das Greenpeace-Schlauchboot auf hoher See, dass auf illegalen Walfang aufmerksam macht. Der Hambacher Wald als Symbol der deutschen Klimabewegung vermag genau das. Er befindet sich unmittelbar neben dem größten Braunkohletagebau auf bundesdeutschem Gebiet. Die Mondlandschaft und die übernatürlich anmutenden Schaufelradbagger von RWE stehen dabei in starkem Kontrast zu den 200 Hektar Wald am Grubenrand. Dieser ist ökologisch äußerst wertvoll und beherbergt unter anderem seltene Tierarten wie die Bechsteinfledermaus. Es ist daher kein $\mathrm{Zu}$ fall, dass sich die deutsche Klimabewegung den Hambacher Forst als Protestort ausgesucht hat, denn er lässt sich symbolisch wirkungsvoll nutzen. Der Schaufelradbagger, der sich an das Naturidyll herangräbt, die Landschaft zerstört und gleichzeitig mit der Kohlekraft eine Technologie unterstützt, die für einen Großteil der $\mathrm{CO} 2$ Emissionen Deutschlands verantwortlich ist. Ein Organisator der ersten Klimacamps im Rheinland sprach im Interview davon, dass es ihre Strategie gewesen war, in der Nähe der Abbruchkante zu campen, um einen visuellen Eindruck von der durch den Tagebau hervorgerufenen Zerstörung zu vermitteln und auf diese Weise das abstrakte Thema Klimawandel besser "packen“ zu können (Interview BUNDJugend NRW 2019: 448ff.). Die Fokussierung auf lokale Großemittenten von $\mathrm{CO} 2$ und weg von Gipfelprotesten geht auf einen Strategiewechsel innerhalb der Klimabewegung zurück, welcher sich nach dem gescheiterten Klimagipfel von Kopenhagen vollzogen hat (vgl. Sander 2016:21). Und da bot sich der Tagebau Hambach mit seinem „Märchenwald“ (Köppe 2018) eher an, als etwa der Tagebau Garzweiler, der vor allem von landwirtschaftlichen Flächen umgeben ist.

Ein erster und wichtiger Verdienst des Waldspaziergangs ist es daher, dass er klimabewegte Menschen in regelmäßigen Abständen an diesem symbolischen Ort versammelt. Er kann das auch deshalb 
tun, weil der Wald strategisch günstig gelegen ist. So liegt er inmitten eines mitteleuropäischen Ballungsraumes direkt neben der Autobahn A4 und ist von Köln aus problemlos mit der S-Bahn zu erreichen (vom Kölner Hbf bis zum S-Bahnhof Buir dauert es 35 Minuten). Das ist gerade im Vergleich zum Lausitzer Braunkohlerevier ein strategischer Vorteil.

\section{Der Waldspaziergang als niedrigschwelliges Angebot}

Michael Zobel, der die Rolle des Waldführers einnimmt, führt Interessierte einmal im Monat durch den Hambacher Wald. Als studierter Geologe und ausgebildeter Naturführer, hat er von Berufswegen eine enge Beziehung zu Wald und Natur (Interview Michael Zobel 2019: 65-68). Nach eigenen Angaben führte jedoch erst ein mehrtägiger Aufenthalt im Hambacher Forst und ein Treffen mit Aktivist*innen 2014 dazu, dass er begann, sich Gedanken über politisches Engagement zu machen (Interview Michael Zobel 2019: 77ff.). Auf eine Idee seiner Lebensgefährtin Eva Töller hin, entschloss er sich ab Mai 2014, einmal im Monat einen von ihm angeleiteten Spaziergang im Hambacher Wald anzubieten. Dieses mit zunächst 50 Spaziergänger*innen gestartete Projekt dauert bis heute an. Das Organisationsteam führt jedes Mal eine Zählung der Teilnehmer*innen durch und dokumentiert diese akribisch, auch um den Erfolg und das Wachstum des Kohleprotests zu unterstreichen. So sagte Michael Zobel im für die Studie durchgeführten Interview, sie könnten demnächst „Teilnehmer oder Teilnehmerin 60.000 begrüßen“ (Interview Michael Zobel 2019: 40). Thematisch geht es bei den Führungen einerseits um scheinbar unpolitische Themen wie die Flora und Fauna des Waldes, den Kohlebergbau sowie andererseits um den sich dagegen formierenden Widerstand. Auch der Besuch eines Baumhausdorfes (ein sogenannter „Barrio“ im Sprachgebrauch der Waldbewohner* innen) ist fester Teil des Programms. Ich möchte im Folgenden argumentieren, dass das Format Waldspaziergang deshalb zum Bewegungsaufbau beitragen konnte, weil es erstens so niedrigschwellig konzipiert ist, dass alle Gruppierungen daran teilnehmen können und zweitens von Seiten der Organisator ${ }^{*}$ innen gezielt Kommunikationsbarrieren abgebaut werden.

Andere Aktionsformate der Klimabewegung wie Klimacamps oder Aktionen massenhaften zivilen Ungehorsam (wie sie von Ende Gelände jährlich organisiert werden) sind zwar ebenfalls ideologisch niedrigschwellig konzipiert, richten sich jedoch primär an ein junges Publikum. Im Gegensatz dazu sind die Teilnehmer*innen der monatlichen Waldspaziergänge generationell relativ durchmischt. Die Palette reicht dort von jungen Menschen im studentischen Alter über junge Familien 
mit Kindern bis hin zu Vertreterinnen älterer Jahrgänge. Auch Menschen aus der autonomen Szene dürfen mit Gesichtsvermummung an dem Waldspaziergang teilnehmen. Diese Offenheit liegt unter anderem darin begründet, dass die Teilnahme an einem Waldspaziergang deutlich weniger zeitliches Engagement erfordert als die Teilnahme an einem Klimacamp und wesentlich weniger persönliche Risikobereitschaft als die Teilnahme an einer Aktion zivilen Ungehorsams.

Außerdem spielt die Wahl des Namens „Waldspaziergang“ und die Figur von Michael Zobel als Repräsentant nach außen eine wichtige Rolle. Zunächst suggeriert der Name einen eher unpolitischen Charakter der Veranstaltung und weckt bei möglichen Teilnehmer*innen die in Deutschland in Verbindung mit Wald und Natur üblichen positiven Assoziationen (vgl. Radkau 2011: 69ff.). Des Weiteren verkörpert Michael Zobel seinem äußeren Erscheinungsbild und der Selbstdarstellung zufolge keinen Öko-Aktivisten, sondern eher einen umweltverbundenen Bürger (Auf seiner Homepage nennt er sich „Naturführer und Waldpädagoge“). Zwar macht er keinen Hehl daraus, dass er sich für den Erhalt des Waldes und gegen die Braunkohle einsetzt, doch trotzdem präsentiert er die Positionen der Waldbesetzung sowie die vorangebrachten Argumente mit einer gewissen Distanz und sieht sich selbst eher als „Vermittler“ im Konflikt.
Und ich hab schon besondere Fähigkeiten, was es so angeht, Menschen zu mobilisieren, Menschen was zu zeigen und zu vermitteln. Und das mach ich. Das ist meine Rolle in diesem ganzen Thema [...].

(Interview Michael Zobel 2019: 85f.)

\section{Von kultureller Distanz zu geschätzter Verschiedenheit}

Aus dieser Offenheit gegenüber dem radikalen und dem bürgerlichen Lager der Bewegung erwächst die Möglichkeit, beide im Rahmen des Formats Waldspaziergang gemeinsam zu versammeln. Dabei können durch die Begegnung und die direkte Kommunikation untereinander Vorurteile beziehungsweise eine kulturelle Distanz zwischen den Akteur*innen abgebaut oder zumindest gemindert werden. Einige Beispiele wären hier zu nennen.

Zunächst bietet der Hambacher Forst für viele Vertreter*innen des sogenannten bürgerlichen Lagers ein eher befremdliches Bild und einem direkten Kontakt mit der Waldbesetzung steht Einiges im Weg. So sind an einigen Waldeingängen Stapel aus Müll und sonstigem Material zu finden, die von der Waldbesetzung als Barrikaden genutzt werden. Außerdem hängen an einigen Stellen im Wald Banner mit politischen Botschaften, die für viele Teilnehmer*innen der Waldspaziergänge eher befremdlich 
anmuten dürften (zum Beispiel „Kapitalismus abschaffen, Staaten überwinden, Anarchismus organisieren"; vgl. Aktion Unterholz 2018: 9). Und schließlich zeigen sich viele der Waldbewohner*innen nur mit vermummtem Gesicht, was bei Menschen, die nicht in der anarchistisch-autonomen Szene sozialisiert sind, eher negative Assoziationen hervorruft und mit dem zum Teil medial vermittelten Image der Aktivist* innen als linksradikale Gewalttäter*innen übereinstimmt (An dieser Stelle möchte ich explizit darauf hinweisen, dass es vor allem im Vorfeld der Räumungen auch sehr viel wohlwollende Medienberichterstattung zur Waldbesetzung gab und Berichte über Gewalttätigkeit immer nur einen Teil davon ausmachten; siehe: Frigelj 2018; Beissel et al. 2016). Für jene Menschen, die zwar mit dem grundsätzlichen Anliegen eines zügigen Kohleausstiegs einverstanden sind, jedoch eine kulturelle Distanz zur Außendarstellung der Waldbewohner*innen empfinden, bietet der Waldspaziergang die Möglichkeit, in einem geschützten Format mit jenen Menschen persönlich und unverbindlich ins Gespräch zu kommen. Dabei spielt Michael Zobel als Moderator des Gesprächs eine wichtige Rolle. Bereits vor dem Besuch des Baumhausdorfes, erklärt er den Teilnehmer*innen die Bedeutung der Barrikaden im Wald, spricht davon, dass sich diese nicht gegen Fußgänger* innen, sondern gegen das Eindringen von Rodungsmaschinen in den Wald richteten. Dadurch konterkariert er den Diskurs von
RWE mit einer Lesart, die der Waldbesetzung prinzipiell wohlgesonnen ist. Ebenso betont er häufiger, dass er bei weitem nicht mit allem einverstanden sei, was die Aktivist ${ }^{*}$ innen rund um den Wald tun. Im gleichen Atemzug betont er aber auch, dass RWE mit dem Tagebau Hambach deutlich größeres Unrecht begehe. Damit fördert er ein Gesprächsklima, dass den Wert der Unterschiedlichkeit der Menschen betont, jedoch die Bekämpfung der Klimakrise als einendes Ziel in den Vordergrund stellt.

Neben der „Vorbereitung“ durch den Waldführer spielt die direkte Interaktion zwischen Besucher*innen und Waldbewohner ${ }^{\star}$ innen eine ebenso wichtige Rolle für das Knüpfen neuer Verbindungen. So kann bei dem Besuch des Barrios über mögliche Streitfragen kontrovers diskutiert werden und bürgerliche Umweltschützerinnen bekommen auf diese Weise ein nuancierteres Bild von den „Menschen aus dem Wald“. So kommt es vor, dass sich die Meinung mancher Spaziergänger ${ }^{*}$ innen durch den direkten Kontakt mit den Aktivist ${ }^{\star}$ innen ändert. Diese sind dann nicht mehr die "Chaoten", sondern authentische Idealisten, mit denen man zwar nicht immer einer Meinung ist, dessen Einsatz für die gute Sache man aber grundsätzlich schätzt. 


\section{Eine durch Begegnung und Diskurs erzeugte Bewegung}

Schlussendlich spielt es bei den Waldspaziergängen aber auch eine wichtige Rolle, dass die einzelnen am Widerstand gegen die Braunkohle beteiligten Gruppierungen nicht nur daran teilnehmen können, sondern dass diesen auch für ihren jeweiligen Beitrag zum Schutz des Waldes Anerkennung zugesprochen wird. Genau das tut Michael Zobel während seinen Waldführungen. So führte er bei einem Waldspaziergang im April 2019 aus, auf welche Weise die verschiedenen Akteur*innen dazu beigetragen haben, dass der Wald möglicherweise erhalten bleibt. Auf der einen Seite hob er den BUND heraus, der auf dem Rechtsweg mit Klagen alles dafür getan habe, einen Rodungsstopp zu erwirken. Ebenso sei die Arbeit der Bürgerinitiative aus dem Tagebauranddorf Buir von Bedeutung, die mit hohem Durchhaltevermögen im Revier „die Stellung halten“ (Der Waldspaziergang ist auf Video festgehalten und bei youtube hochgeladen worden: www.youtube.com/ watch?v=b5WuHhFEgQU, zuletzt geprüft am 01.06.2020). Durch diese Würdigung der jeweiligen Beiträge zur gemeinsamen Sache, vermag es Michael Zobel bei den Waldspaziergängen eine Bewegung zu kreieren, deren Bestandteile sich einzeln ohne dieses Verknüpfungsformat wohl kaum dazu bekennen würden. Das Beispiel des Hambacher Waldes zeigt, dass es für das
Formieren von Protestnetzwerken nicht zwangsläufig vonnöten ist, über eine gemeinsame Identität zu verfügen. Vielmehr reicht ein gemeinsames Handlungsformat, an dem alle Einheiten gleichermaßen teilnehmen können. Oder anders formuliert: Der Waldspaziergang befördert die Herausbildung einer Bewegungsidentität, deren Wert insbesondere in der Unterschiedlichkeit und der Komplementarität ihrer Bestandteile begründet liegt.

\section{Stabilität durch Übersetzung}

Das Beispiel Hambacher Forst zeigt, dass die kollektive Dimension in sozialen Bewegungen nicht als gegeben vorausgesetzt werden kann. Ebenso wenig erscheint es vor diesem Hintergrund plausibel, einer zu engen Begriffsdefinition von sozialen Bewegungen anzuhängen und jene Netzwerke und Koalitionen a priori auszuschließen, die eventuell keine eindeutige kollektive Identität aufweisen. Vielmehr lädt uns das Beispiel Hambach ein, die Übersetzungsarbeit nachzuvollziehen, die geleistet werden muss, um nicht-assoziierte Entitäten $\mathrm{zu}$ einem Protestnetzwerk zu verbinden. Die Prozesse, mittels derer sich diese Verbindung vollzieht, gilt es wie im Falle des Waldspaziergangs zu beleuchten und in ihrer Bedeutung einzuschätzen. Michel Callon spricht in diesem Zusammenhang von einem mehrstufigen Übersetzungsprozess der imstande ist - ausgehend von 
einer Problemdefinition des Hauptakteurs (in diesem Fall die Organisator*innen des Waldspaziergangs) - neue Verbündete in das Netzwerk zu integrieren, unter den Verbündeten Verhandlungen zu führen und das Netzwerk schließlich nach außen $\mathrm{zu}$ stabilisieren (Die vier Stufen heißen problematization, interessement, enrolment, mobilization; vgl. Callon 1986; Schritt 2019: 144). Der Waldspaziergang ist jedoch nur eines von mehreren Versammlungsformaten, die dem Netzwerk heterogener Akteur*innen Stabilität verleihen konnte. dende Klimacamps und Aktionen zivilen Ungehorsams, die die aktionsorientierte Dimension der Bewegung stärker in den Mittelpunkt rücken. Ich habe mich im Rahmen dieses Artikels dafür entschieden, die Funktion des Waldspaziergangs für die Kohleproteste am Hambacher Forst zu untersuchen, weil es dieses Format war, dass während der Räumungen im September 2018 einen erheblichen Konjunkturschub erhielt. Plötzlich wurden die monatlichen Waldführungen einmal in der Woche veranstaltet und an ihnen nahmen mehrere tausend Menschen teil (an einem Wochenende im September waren es nach Aussage von Michael Zobel 15.000 Menschen, Interview Michael Zobel 2019: 789).

Anhand der Proteste gegen die Rodung des Hambacher Forstes lässt sich der Wert jener sozialen Bewegungen herausstellen, die auf niedrigschwelligen Verknüpfungen

von äußerst unterschiedlichen Akteur*innen beruhen. Dadurch, dass Formate wie der Waldspaziergang sowohl Umweltverbände als auch Teile der anarchistischen Waldbesetzung an einem gemeinsamen Ort versammeln können, ist es der „Bewegung Hambacher Forst" möglich gewesen, die sehr unterschiedlichen Ressourcen der jeweiligen Gruppierungen im Sinne einer aktivistischen Arbeitsteilung für die gemeinsame Sache einzubringen. Denn nur ein anerkannter Naturschutzverein wie der BUND NRW hat die Befugnis gegen die Betriebspläne von RWE zu klagen und auf diese Weise einen temporären Rodungsstopp zu erwirken. Auf der anderen Seite verfügen vor allem informelle Aktivist*innenkollektive über das notwendige Know-how, um Sitzblockaden und sonstige Aktionen im Zusammenhang mit den Räumungen zu organisieren sowie öffentlichkeitswirksam zu inszenieren. Dass diese zwei Pole der Bewegung jedoch als zwei Seiten desselben Braunkohlewiderstands wahrgenommen werden können und nicht automatisch als guter und schlechter Aktivismus gelabelt werden, liegt auch an den seit Jahren bestehenden Formaten der Begegnung und des Diskurses.

\section{Das positive Symbol der deutschen Klimabewegung}

Auf gesamtgesellschaftlicher Ebene hat die "Bewegung Hambacher Forst" einen 


\section{9 \\ Auf gesamtgesellschaftlicher Ebene hat die „Bewegung Hambacher Forst“ einen Beitrag dazu geleistet, dass vorhandenes Wissen über Risiken der Erderwärmung in ein konkretes, politisches Handlungsprogramm zu dessen Bekämpfung übersetzt wurde.}

Beitrag dazu geleistet, dass vorhandenes Wissen über Risiken der Erderwärmung in ein konkretes, politisches Handlungsprogramm zu dessen Bekämpfung übersetzt wurde. Der Konflikt hat die Debatte über einen zeitigeren Ausstieg aus der Braunkohle angeregt und mit dem Wald gleichzeitig ein positives Symbol für die deutsche Klimabewegung geschaffen. So sah sich auch die nur wenige Monate vor dem Polizeieinsatz eingesetzte sogenannte „Kohlekommission“ genötigt, in ihrem Abschlussbericht zu vermerken, der Erhalt des Hambacher Forstes sei „wünschenswert“ (Kommission Wachstum Strukturwandel Beschäftigung 2019: 63). Die Auseinandersetzung um den Hambacher Forst konnte auf diese Weise zu einem heroischen Kampf stilisiert werden, auf den sich viele andere Akteur*innen der Klimabewegung gerne berufen. So widmete Greta Thunberg den Gewinn des Fernsehpreises „Goldene Kamera“ den Aktivist ${ }^{\star}$ innen aus dem Hambacher Forst und auch bei den aufkommenden Fridays-for-Future-Demonstrationen waren Symbolik und Slogans aus dem Hambacher Forst präsent.
Es scheint, als verschaffe sich eine Deutung der Klimakrise mehr Raum, welche die systemische Frage nach einer Transformation des Energieregimes stellt und nicht ausschließlich auf Fragen des individuellen Konsums begrenzt bleibt. Einige Interviewpartner ${ }^{*}$ innen sprachen von einer Diskursverschiebung zu ihren Gunsten (vgl. Interview Ende Gelände 2019: 10981104) und wiederum ein Vertreter der IG BCE kritisierte die Hegemonie des "grünen Mainstreams" in Medien und Öffentlichkeit (vgl. Interview IG BCE 2019: 435). Die Themen der Klimabewegung verfügen im öffentlichen Diskurs über hohe Anerkennung, jedoch bleiben die von der Regierung getroffenen Maßnahmen bisher weit hinter den Forderungen zurück, was sich besonders prägnant am späten Ausstiegsdatum für die Braunkohle festmachen lässt.

Um dieses Paradoxon aus der Perspektive der Soziologie der Übersetzung erklären zu können, ließe sich sagen, dass die mittels Übersetzung geknüpften Verbindungen anscheinend noch nicht umfassend genug sind, um einen radikalen Strategiewechsel 
mehrheitsfähig zu machen. Ebenso könnte man hinzufügen, dass der Themenkomplex der globalen Erderwärmung und die Frage nach den geeigneten Strategien zu deren Bekämpfung von einer starken Disparität zwischen Anerkennung des Problems im Allgemeinen und Uneinigkeit der Lösungsstrategien im Konkreten geprägt sind. So verweisen Politiker*innen fast aller Parteien auf die Pariser Klimaziele als positive Referenz und sehen grundsätzlich die Notwendigkeit einer ambitionierten Klimapolitik. Allerdings werden die von gen (Dekarbonisierung des Energiesektors, Verkehrswende, Agrarwende) beispielsweise von Vertreter ${ }^{\star}$ innen der Industrie oder von konservativ-liberalen Parteien mit dem Verweis auf „nationale Alleingänge" (vgl. Interview IG BCE: 64-74) oder „planwirtschaftliche Eingriffe in den Energiemarkt" (FDP 2016: 1) abgelehnt. Es lässt sich daher schlussfolgern, dass soziale Bewegungen wie der Hambacher Forst auch deshalb so viel wohlwollende Aufmerksamkeit und Anerkennung erfahren, weil sie die Erkenntnis des menschengemachten Klimawandels primär in ein allgemeines Problembewusstsein übersetzen.

\section{LITERATUR}

Aktion Unterholz (2018): „Mama, dieser Stock auch?“. Von Barrikaden, Waldschützer*innen und Antikapitalismus. Broschüre der Aktion Unterholz.

Beck, Ulrich (2016 [1986]): Risikogesellschaft. Auf dem Weg in eine andere Moderne. Frankfurt am Main: Suhrkamp.

Beissel, Udo/Rupprecht, Bernd/Jansen, Ralph (2016): Eine neue Stufe der Gewalt. In: Kölner Stadtanzeiger, 23.01.2016.

Benford, Robert/Snow, David A. (2000): Framing Processes and Social Movements: An Overview and Assessment. In: Annual Review of Sociology Jg. 26, S. 611-639.

Bosse, Jana (2015): Kein Land mehr für Kohle - Kohleausstieg ist Handarbeit. In: Forschungsjournal Soziale Bewegungen Jg. 28/4, S. 394-399.

Brand, Karl-Werner (2014): Umweltsoziologie. Entwicklungslinien, Basiskonzepte und Erklärungsmodelle. Weinheim: Beltz Juventa.

Brock, Andrea/Dunlap, Alexander (2018): Normalising corporate counterinsurgency: Engineering consent, managing resistance and greening destruction around the Hambach coal mine and beyond. In: Political Geography Jg. 62 , S. 33-47.

Callon, Michel (1986): Some elements of a sociology of translation. Domestication of the scallops and the fishermen of St Brieuc Bay. In: John Law (Hrsg.): Power, action and belief: a new sociology of knowledge? London: Routledge \& Kegan Paul, S. 196-233.

Eyermann, Bernd (2018): Hambacher Forst als Symbol. Steinkohleförderung in NRW ist Geschichte, über Braunkohleausstieg wird gestritten. In: Kölnische Rundschau, 31.12.2018.

Freie Demokratische Partei (FDP) (2016): Beschluss des Bundesvorstands der FDP. Für eine zukunftsfähige Klimapolitik. Berlin. Online verfügbar unter https://www.fdp. de/sites/default/files/uploads/2016/06/08/20160606buvofuereinezukunftsfaehigeenergiepolitikneu.pdf (08.09.2020).

Frigelj, Kristian (2018): Radikale auf den Bäumen. In: DIE WELT, 04.09.2018, S. 6. 
Hagemann, Ingmar/Vey, Judith/Leinius Johanna (Hrsg.) (2019): Handbuch Poststrukturalistische Perspektiven auf soziale Bewegungen. Bielefeld: transcript.

Hale, Charles R. (2008): Engaging contradictions. Theory, politics, and methods of activist scholarship. Berkeley: University of California Press

Hambacher Forst Buchprojekt (2015): Mit Baumhäusern gegen Bagger. Geschichten vom Widersand im rheinischen Revier. Osnabrück: Packpapierverlag.

Infratest dimap (2019): Umfragen wahlentscheidende Themen. Online verfügbar unter https://wahl.tagesschau. de/wahlen/2019-05-26-EP-DE/umfrage-wahlentscheidend. shtml (01.06.2019).

Kaufer, Ricardo/Lein, Paula (2018): Widerstand im Hambacher Forst. Analyse einer anarchistischen Waldbesetzung. In: Forschungsjournal Soziale Bewegungen Jg. 31/4, S. 1-12.

Kommission „Wachstum, Strukturwandel und Beschäftigung“ (2019): Abschlussbericht. Frankfurt am Main: Druck- und Verlagshaus Zarbock. Online verfügbar unter https://www.bmwi.de/Redaktion/DE/Downloads/A/ abschlussbericht-kommission-wachstum-strukturwandel-und-beschaeftigung.pdf? blob=publicationFile (12.07.2020).

Köppe, Julia (2018): Der Märchenwald. In: Spiegel Online. Online verfügbar unter https://www.spiegel. de/wissenschaft/natur/hambacher-forst-der-maerchenwald-a-1241613.html\# (21.09.2020).

Latour, Bruno (2014): Eine neue Soziologie für eine neue Gesellschaft. Einführung in die Akteur-Netzwerk-Theorie. Frankfurt am Main: Suhrkamp.

Mathieu, Lilian (2012): L'espace des mouvements sociaux. Bellecombe-en-Bauges: Éditions du Croquant.

McAdam, Doug (1982): Political process and the development of black insurgency, 1930 - 1970. Chicago: The University of Chicago Press.

McCarthy, John D./Zald, Mayer N. (1977): Resource Mobilization and Social Movements: A Partial Theory. In: American Journal of Sociology Jg. 82/6, S. 1212-1241.

Parth, Christian (2018): Das große Waldfest. In: Kölner Stadtanzeiger, 08.10.2018.
Radkau, Joachim (2011): Die Ära der Ökologie. Eine Weltgeschichte. München: C.H. Beck.

Roth, Roland/Rucht, Dieter (Hrsg.) (2008): Die sozialen Bewegungen in Deutschland seit 1945. Ein Handbuch. Frankfurt am Main: Campus.

Sander, Hendrik (2016): Die Klimagerechtigkeits-Bewegung in Deutschland. Entwicklungen und Perspektiven. Berlin: Studien der Rosa Luxemburg Stiftung.

Schritt, Jannik (2019): Contentious Assemblages. Gefüge, Affekt, politische Situationen und die erweiterte Fallmethode als Analysewerkzeuge zum Verständnis urbaner Aufstände. In: Hagemann, Ingmar/Vey Judith/Leinius, Johanna (Hrsg.): Handbuch Poststrukturalistische Perspektiven auf soziale Bewegungen. Bielefeld: transcript, S. 138-151.

Tarrow, Sidney G. (2005): The new transnational activism. Cambridge: Cambridge University Press.

Thunberg, Greta (2019): Rede vor dem Kongress der USA. Online verfügbar unter https://www.independent.co.uk/ voices/greta-thunberg-congress-speech-climate-change-crisis-dream-a9112151.html (23.06.2020).

\section{ZUM AUTOR}

Ruben Kaiser, 27 Jahre, hat Politikwissenschaft an der Katholischen Universität Eichstätt-Ingolstadt, am Institut d'Etudes Politiques de Rennes sowie an der Université Paris-Nanterre studiert. Er beschäftigt sich schwerpunktmäßig mit der soziologischen Analyse jüngerer Umweltproteste in Deutschland.

An dem Beitrag haben folgende Redaktionsmitglieder im Review, Betreuung und Lektorat mitgearbeitet: Nils Haacke, Andreas Schulz und Luisa Bischoff. 


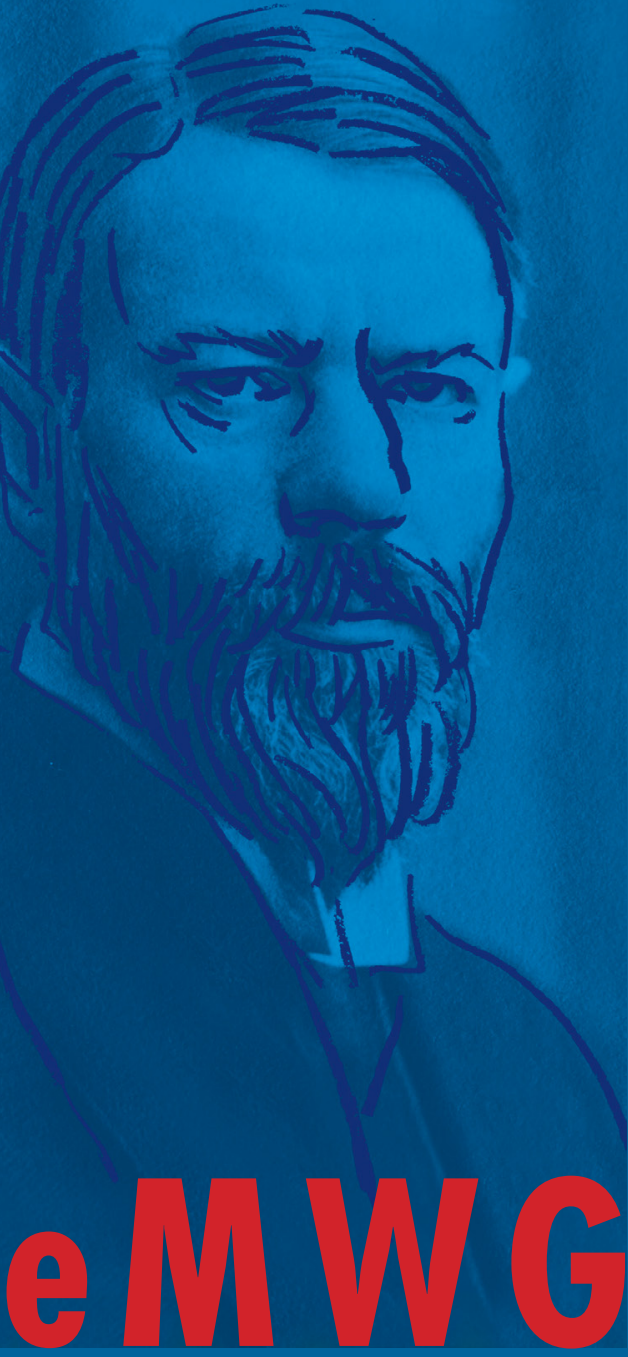

Anlässlich des 100. Todestages von Max Weber veröffentlicht Mohr Siebeck alle Bände der Max WeberGesamtausgabe (MWG) als eBook.

Die eMWG ist jetzt zum Paketpreis mit 30\% Sonderrabatt erhätlich. Einzelbände sind zum Preis der Leinenausgabe lieferbar.

Weitere Informationen und Bestellungen:

László Simon-Nanko (simon-nanko@mohrsiebeck.com).

MOHR SIEBECK mohrsiebeck.com/emwg
Mit dem Erscheinen der Bände II/ 11 und III/2 der Max Weber-Gesamfausgabe wird die Edition nach ihrem Beginn im Jahre 1984 nun erfolgreich abgeschlossen.

Band II/11:

Briefe. Nachträge und Gesamtregister

Herousgegeben von Rita Aldenhoff-Hübinger

und Edith Hanke

2019. XXVI, 707 Seiten (MWG II/11).

Einzelbezug: ISBN 978-3-16-155603-6 Leinen € 319,-

Band III/2:

\section{Praktische Nationalökonomie}

Vorlesungen 1895-1899

Herausgegeben von Hauke Janssen

in Zusammenarbeit mit Cornelia Meyer-Stoll

und Ulich Rummel

2020. Ca. 450 Seiten (MWG III/2).

Einzelbezug: ISBN 978-3-16-153080-7 Leinen € 234,(erscheint im Juni)

\section{Außerdem sind neu erschienen:}

Max Weber

\section{Gelehrtenbriefe}

1878-1920. Ausgewählte Briefe II

Hercusgegeben von Rita Aldenhoff-Hübinger

und Edith Hanke

Mit einem Einleitungsessay von Gangolf Höbinger

2020. XXVVI, 267 Seiten. ISBN 978-3-16-157516-7

Festeinband $€ 29$,-

Max Weber

\section{Wissenschaft als Beruf/Politik als Beruf}

Jubiläumsausgabe

Hercusgegeben von Wolfgang J. Mommsen

und Wolfgang Schluchter, in Zusammenarbeit

mit Birgitt Morgenbrod

2020. XIII, 296 Seiten (utb fü Wissenschaft Stuttgart).

ISBN 978-3-8252-5000-3 Broschur $€ 15$,- 


\title{
Populismus und Klimaschutz
}

\section{Der AfD-Klimadiskurs}

von Georg Sturm

\begin{abstract}
Die Auseinandersetzung um die Ausgestaltung des Klimaschutzes bestimmt spätestens seit dem Aufkommen der „Fridays for Future“-Bewegung einmal mehr die öffentliche Debatte in Deutschland. Als Reaktion auf die Klimabewegung hat die Alternative für Deutschland (AfD) den Kampf gegen den Klimaschutz zur neuen Hauptaufgabe ihrer Partei auserkoren. Ziel dieses Beitrags ist es - unter Rückgriff auf ökonomische, kulturelle und politische Erklärungsansätze für das Erstarken autoritär-populistischer Parteien - zu untersuchen, inwiefern die AfD mit ihrem Klimadiskurs populistisches Protestpotenzial adressiert. Die qualitative und quantitative Auswertung der AfD-Pressemitteilungen zu diesem Thema zeigen, dass die AfD in ihrem Anti-Klimadiskurs in erster Linie die Angst vor ökonomischen Einbußen schürt und soziale Abstiegsängste ihrer (potenziellen) Wählerinnenschaft anspricht, sich aber auch an das aus einem kulturellen Wertewandel und politischer Entfremdung resultierende, autoritär-populistische Protestpotenzial richtet. Aus dieser Erkenntnis werden Empfehlungen für eine Klimaschutzpolitik abgeleitet, welche die Akzeptanz für Klimaschutzmaßnahmen erhöhen könnten.
\end{abstract}

\section{Schlagwörter}

Populismus; Klimaschutz; Alternative für Deutschland 


\section{Einleitung}

In einem Gespräch mit der „Welt" erklärte der Parteivorsitzende der „Alternative für Deutschland“ (AfD) Alexander Gauland im September 2019 den Kampf gegen den Klimaschutz zur neuen Hauptaufgabe seiner Partei: „Die Kritik an der sogenannten Klimaschutzpolitik ist nach dem Euro und der Zuwanderung das dritte große Thema für die AfD.“ (zitiert nach Kamann 2019) Während die Auseinandersetzung um den Klimaschutz spätestens seit dem Aufkommen der „Fridays for Future“-Bewegung die öffentliche Debatte in Deutschland bestimmt, versucht die AfD einen Gegendiskurs zu etablieren, indem sie sowohl den Klimawandel an sich infrage stellt als auch die Klimapolitik der Bundesregierung und die klimapolitischen Positionen anderer Akteurinnen grundsätzlich kritisiert („Klimahysterie“; Meuthen 2019).

Die mit der Energiewende und dem Klimaschutz einhergehenden Transformationsprozesse sind aus sozialwissenschaftlicher Perspektive von erheblicher Relevanz, da sie zeigen, wie gesellschaftliche Aushandlungs- und Verarbeitungsprozesse ablaufen. Neben klassischen energiepolitischen Analysen im Kontext der Energiewendeforschung wird hierbei vermehrt auf Ansätze aus der Diskurs-, Lebenswelt- und sozialen Bewegungsforschung zurückgegriffen. Der politische und gesellschaftliche Kontext der Energiewende wird im
Folgenden als „ideale Vorlage für Kritik von populistischen Bewegungen" und ein Spiegelbild gesellschaftlicher Stimmungslagen angesehen (Radtke et al. 2019: 5f.).

Ziel dieses Beitrags ist es, zu untersuchen, inwiefern die AfD mit ihrem Klimadiskurs populistisches Protestpotenzial adressiert. Hierbei wird von einem Diskursbegriff ausgegangen, nach dem soziale Akteur ${ }^{*}$ innen sowohl als diskursiv konstituierte Handelnde als auch als aktive Produzent ${ }^{\star}$ innen und Rezipient*innen von Diskursen in Erscheinung treten (vgl. Keller 2008: 12). Da das Forschungsinteresse dieses Beitrags darin besteht, zu untersuchen, wie die AfD ein bestimmtes Klima-Narrativ strategisch einsetzt, liegt der Fokus weniger auf dem Aspekt, wie Subjekte diskursiv produziert werden, sondern vielmehr auf der Rolle handelnder Akteur ${ }^{\star}$ innen im Prozess der Diskursproduktion. Dadurch soll die Frage beantwortet werden, warum die AfD dieses Thema zur neuen Hauptaufgabe der Partei erklärt hat. Unter Rückgriff auf ökonomische, kulturelle und politische Erklärungsansätze für das Erstarken autoritär-populistischer Akteur*innen (vgl. Zürn 2018) wird abschließend diskutiert, welche der Erklärungsansätze die von der AfD eingenommene Haltung und diskursive Strategie zu diesem spezifischen Thema am besten erklären.

In einem ersten Schritt werden der Begriff des (autoritären) Populismus theoretisch 
konzeptualisiert und die drei zentralen Erklärungsansätze für dessen Erstarken skizziert. Die ökonomische Unsicherheitsperspektive (vgl. Manow 2018; Kohlrausch 2018), der kulturelle Wertewandel (Inglehart/Norris 2016; de Wilde et al. 2019) und die politische Entfremdung (Zürn 2018) dienen als Konzepte, aus denen deduktive Oberkategorien für die kategoriengeleitete qualitativ-orientierte Textanalyse abgeleitet werden. Mithilfe dieser wird AfD-Klimadiskurs auf Grundlage der Pressemitteilungen des AfD-Bundesvorstands zum Thema „Klima“ aus dem Jahr 2019 in einem zweiten Schritt inhaltsanalytisch untersucht und im Hinblick auf die verschiedenen Erklärungsansätze sowohl interpretativ als auch quantitativ ausgewertet.

Mithilfe der Ergebnisse wird abschließend die Frage beantwortet, welche Erklärungsmuster die Auswahl dieses Themas als Hauptaufgabe der Partei plausibel begründen können. Zudem wird diskutiert, welche politischen Lehren aus diesen Forschungsergebnissen gezogen werden können, um Klimaschutzpolitik gegen Angriffe autoritär-populistischer Akteurinnen zu wappnen und die gesellschaftliche Akzeptanz für Klimaschutz-Maßnahmen zu erhöhen.

\section{Autoritärer Populismus und seine Erklärungsansätze}

Für den niederländischen Politikwissenschaftler Cas Mudde (2004) ist Populismus von zwei zentralen Merkmalen gekennzeichnet: Zum einen teilt diese Ideologie die Gesellschaft in zwei in sich homogene und antagonistische Gruppen ein - ,the pure people“ auf der einen und "the corrupt elite" auf der anderen Seite (Mudde 2004: 543). Zum anderen unterstellt der Populismus, es gäbe den einen „volonté générale“, dessen Umsetzung er für sich beansprucht (ebd.). Als „dünne“ Ideologie ("thin-centred ideology“) habe Populismus einen nur begrenzten ideologischen Kern (Freeden 1998: 750), der sich auf der Grundlage der beiden genannten Merkmale mit unterschiedlichen „host ideologies“ verbinden lässt. Innerhalb der verschiedenen Definitionen in der Populismusforschung eignet sich ein solcher Populismusbegriff am besten, da er Populismus nicht nur auf seine politisch-kommunikative Strategie reduziert (wie dies etwa Jagers/Walgrave 2007; Bonikowski 2017 tun), sondern auf dessen („dünnen“) ideologischen Kern abzielt, der von Anti-Elitismus und Volkszentriertheit gekennzeichnet ist.

Da diese Definition verschiedene Ausprägungen des Populismus umfasst und der verbreitete Begriff des Rechtspopulismus zur Charakterisierung der AfD 
verharmlosend und aufgrund seiner häufigen Nutzung als politischer Kampfbegriff unpräzise ist, wird im Folgenden auf das Konzept des völkisch-autoritären Populismus des Sozialwissenschaftlers Alexander Häusler (2019) zurückgegriffen. Dessen Charakterisierung ist geeignet, da sie richtigerweise feststellt, dass die AfD als parteipolitisches Dach verschiedener Milieus sowohl (rechts)populistische als auch autoritäre, völkisch-nationalistische und extrem rechte Positionen und Strategien in sich vereint (ebd.: 83ff.).

\subsection{Populismus und Demokratie}

Das Verhältnis des Populismus zur Demokratie ist Gegenstand einer kontroversen Debatte in der Politischen Theorie und Populismusforschung. Vertreter ${ }^{\star}$ innen eines deliberativen Demokratieverständnisses stellen eher die negativen und demokratiegefährdenden Aspekte des (autoritären) Populismus in den Vordergrund. Ihnen zufolge ist der Populismus anti-liberal, anti-pluralistisch und anti-multilateral (vgl. Zürn 2018: 3).

Anhänger ${ }^{*}$ innen eines antagonistischen Demokratieverständnisses betonen hingegen den demokratisierenden Charakter von populistischen Ausdrucksformen (vgl. Laclau 2018; Mouffe 2018). Der Politologe Philip Manow sieht in dem Erfolg populistischer Akteur ${ }^{*}$ innen keinen Ausdruck der
Krise der Demokratie, sondern vielmehr das Ergebnis einer tiefen Legitimationskrise der demokratischen Repräsentation (vgl. Manow 2020: 21). Diese Überlegung weist daraufhin, dass populistischer Protest als Korrektiv einer niedrigen Responsivität des politischen Systems gegenüber bestimmten Interessen funktionieren kann. Vor diesem Hintergrund dient die Analyse des AfD-Klimadiskurses auch der Identifikation möglicher Repräsentationslücken in der Klimaschutzpolitik. Daraus sollen Empfehlungen abgeleitet werden, wie Klimapolitik eine höhere gesellschaftliche Akzeptanz erlangen kann.

Wieso es in den letzten Jahren zu einem Aufstieg autoritär-populistischer Parteien in Europa kommen konnte, wird in der Politikwissenschaft und Soziologie kontrovers diskutiert. Im Wesentlichen lassen sich die verschiedenen Ausführungen drei zentralen Erklärungsansätzen zuordnen - einer ökonomischen, einer kulturellen und einer politischen Erklärung. Diese werden im Folgenden in ihren Grundzügen skizziert, um daraus Kategorien abzuleiten, die die inhaltsanalytische Untersuchung des AfD-Klimadiskurses strukturieren können.

Hierbei ist es wichtig anzumerken, dass es sich bei der Zustimmung zu autoritärpopulistischen Parteien und Bewegungen um ein vielschichtiges Phänomen handelt, das sich nicht monokausal erklären lässt (vgl. Dörre et al. 2018: 58). Die drei 
zentralen Erklärungen sind - anders als oftmals von den Vertreter*innen der jeweiligen Ansätze unterstellt - durchaus miteinander verwoben. Auf die Interdependenzen der verschiedenen Erklärungsansätze hebt insbesondere Klaus Kraemer (2018: 288) ab, wenn er feststellt, „dass wechselseitige Beeinflussungen oder Steigerungen von kulturellen, sozioökonomischen und soziopolitischen Faktoren möglich sind“. Beispielsweise sei zu vermuten, dass eine stabilere sozioökonomische Position die Gelassenheit steigere, kulturelle Vielfalt im Alltag zu akzeptieren. Auf die Verwobenheit der ökonomischen und der kulturellen Position verweist auch Floris Biskamp und argumentiert, dass die „Ökonomie selbst [...] durch Faktoren mitstrukturiert [sei], die gemeinhin als ,kulturell' verstandenen werden“ (Biskamp 2019: 470). Beispielsweise hingen die meist der kulturellen Sphäre zugeordneten Konfliktfelder rund um Geschlecht und Ethnizität eng mit ökonomischen Fragen zusammen (ebd.: 471).

\section{2 Ökonomische Erklärung}

Die ökomische Erklärung macht in erster Linie die materiellen Folgen des mit der Globalisierung einhergehenden Strukturwandels in den ehemaligen Industriegesellschaften für den Aufstieg des autoritären Populismus verantwortlich. Während die Globalisierung in den letzten Jahrzehnten für einen Anstieg des globalen
Entwicklungsniveaus sorgte, nahm die Ungleichheit innerhalb der westlichen Industrieländer zu (Alvaredo et al.: 13fff.). Dem enormen Einkommensanstieg in der Mittelklasse der Weltgesellschaft, also insbesondere der sogenannten Schwellenländer wie China und Indien, steht eine Stagnation der Einkommen der etablierten Arbeiter ${ }^{*}$ innenschaft in den ehemaligen Industrieländern und ein enormer Zugewinn der oberen Einkommensschichten in diesen Ländern entgegen (vgl. Milanović 2016: 11).

Der Sozialwissenschaftler Oliver Nachtwey (2016) spricht in Anbetracht der Einkommensentwicklung in Deutschland von einer „Abstiegsgesellschaft“. Während der keynesianische Kapitalismus in der "sozialen Moderne" ein hohes jährliches Wirtschaftswachstum und analog zur Entwicklung der Arbeitsproduktivität steigende Nettorealverdienste sowie eine größere soziale Mobilität ermöglichte, nahm dieser „kollektive Fahrstuhleffekt“ (Beck 1986: 124), also der zeitgleiche materielle Zugewinn verschiedener Klassen, in den letzten Jahrzehnten ab. Seit Beginn der 1990er Jahre stiegen die Haushaltseinkommen der oberen sechzig Prozent in Deutschland an, während die unteren vierzig Prozent in diesem Zeitraum Lohnstagnation und -verluste erlebten (Nachtwey 2019: 3). Zudem lässt sich in diesem Zeitraum eine deutliche Zunahme atypischer Beschäftigungsverhältnisse feststellen. Waren im 
Jahr 1991 noch 79 Prozent aller Arbeitnehmer*innen in einem Normalarbeitsverhältnis beschäftigt, sank dieser Anteil auf 68,3 Prozent im Jahr 2014. Im selben Jahr waren 29,9 Prozent der Erwerbstätigen atypisch, beispielsweise befristet, in Teilzeit oder über Leiharbeit, angestellt (ebd.: 3ff.). In einer Studie für die gewerkschaftsnahe Hans-Böckler-Stiftung argumentiert die Soziologin Bettina Kohlrausch (2018: 4), „dass soziale Verunsicherung und soziale Ängste wichtige Treiber der AfD Wahl sind“. Auch wenn Abstiegsängste bei Geringverdiener*innen am stärksten ausgeprägt seien, betreffe die Sorge vor gesellschaftlichem Abstieg auch Personen aus der Mittelschicht mit vergleichsweise hohem Einkommen.

Deren Abstiegsängste resultierten vielmehr aus einem Gefühl des „Ausgeliefertseins“ und der Sorge vor gesellschaftlichen Veränderungen am Arbeitsplatz durch Globalisierung und Digitalisierung speisen (vgl. ebd.: 18, 22). In diesem Gefühl der sozialen Verunsicherung sieht Kohlrausch den Nährboden für den Aufstieg der AfD.

Korte et al. stellen fest, dass die AfD eine „typische ,Defizitpartei“" sei, die für Wähler*innen attraktiv sei, da sie Angebote mache, die bei den etablierten Parteien (vermeintlich) fehlten (2015: 60). Beispielsweise wird vermutet, dass in der ablehnenden Haltung der AfD-Anhänger*innen gegenüber Geflüchteten oftmals
Gerechtigkeitsfragen eine Rolle spielen. Insbesondere in unteren Schichten wird es als ungerecht wahrgenommen, dass bei öffentlichen Ausgaben jahrelang gespart wurde, für Geflüchtete jedoch Geld gezahlt werde. Für diese Personen scheint die AfD eine Lücke zu füllen und „eine Alternative zur Merkelschen Flüchtlingspolitik anzubieten" (Hambauer/Mays 2018: 151). Ein ähnliches Phänomen lässt sich in Bezug auf das Thema Klimaschutz beobachten, wie die folgende Analyse des AfD-Klimadiskurses zeigt.

\subsection{Kulturelle Erklärung}

Während die ökonomische Erklärung die materiellen Folgen des Strukturwandels durch die Globalisierung in den Vordergrund rückt, nehmen die kulturellen Erklärungsansätze den mit dieser Entwicklung einhergehenden Wertewandel in den Blick. Das Aufkommen des autoritären Populismus wird im Wesentlichen als Gegenreaktion auf diesen Wertewandel verstanden, der sich an den Schlagwörtern Post-Materialismus, Multikulturalismus und Feminismus festmacht (vgl. Zürn 2017.: 3f.). Diese Erklärung verweist auf eine neue gesellschaftliche Konfliktlinie, die als Ergebnis der sozialen Revolution der Globalisierung die im 20. Jahrhundert prädominante Auseinandersetzung zwischen Kapital und Arbeit, zwischen Links und Rechts, um eine kulturelle Dimension er- 


\section{9}

Das Aufkommen des autoritären Populismus wird im Wesentlichen als Gegenreaktion auf diesen Wertewandel verstanden, der sich an den Schlagwörtern Post-Materialismus, Multikulturalismus und Feminismus festmacht.

weitert, den Gegensatz zwischen Kosmopolit ${ }^{\star}$ innen und Kommunitarist ${ }^{\star}$ innen (vgl. ebd.: 4).

Der Politikwissenschaftler Michael Zürn sieht im autoritären Populismus und im Neoliberalismus jeweils eine „schmutzige“ Variante (2018: 7) der politischen Ideologien des Kommunitarismus des Kosmopolitismus. Der Gegensatz von Kosmopolit ${ }^{\star}$ innen und Kommunitarist ${ }^{*}$ innen kondensiert sich an der Bewertung der Bedeutung von Grenzen: Während sich Kosmopolit*innen tendenziell für offene Grenzen und Multilateralismus einsetzen, betonen Kommunitarist ${ }^{*}$ innen die ordnungspolitische Ebene des Nationalstaats zur Verwirklichung von Demokratie und Gerechtigkeit (vgl. ebd.: 7f.).

In diesem kulturellen Konflikt stehen sich Globalisierungsgewinner*innen und -verlierer*innen entgegen. Wer von den kulturellen Vorteilen der Globalisierung profitieren kann, hängt stark vom transnationalen Sozialkapital ab (vgl. de Wilde et al. 2019). Während die sogenannten "Anywheres" über höhere Mobilität verfügen, an keinen Ort gebun- den und offen für Veränderung sind, sind die "Somewheres“ - oftmals aufgrund geringerer Bildung und finanzieller Ausstattung - lokal oder regional gebunden und lehnen Veränderung, insbesondere Zuwanderung, ab (vgl. Goodhart 2020: 60ff.). Die darauf basierenden Narrative und Identitätskonzepte betonen die Bedeutung des Nationalen und der Heimat, was als Abwehrreaktion auf eine sich verändernde globalisierte Welt verstanden werden kann. Der kulturelle Liberalismus und der damit verbundene Wertewandel („silent revolution“) sind in westlichen Gesellschaften so dominant geworden, dass dies zu einer aggressiven Gegenreaktion einstmals kulturell dominanter Sektoren geführt hat, die eine Erosion ihrer Privilegien und ihres Status befürchten (Inglehart/Norris 2016: 3).

\subsection{Politische Erklärung}

Die politische Erklärung für den Aufstieg des autoritären Populismus, die insbesondere von Michael Zürn (2018) vertreten wird, baut auf dieser neuen gesellschaftlichen Konfliktlinie zwischen 
Kosmopolit*innen und Kommunitarist ${ }^{\star}$ innen auf. Sie bezieht diese auf durch die strukturellen Entwicklungen der letzten Jahrzehnte veränderten Prozesse der politischen Entscheidungsfindung. Aus einer historisch-institutionalistischen Perspektive stellt dieser Erklärungsansatz eine zunehmende Verbreitung nicht-majoritärer Institutionen - wie Zentralbanken, Verfassungsgerichte oder internationale Organisationen - in konsolidierten Demokratien fest (vgl. Zürn 2017).

Innerhalb dieser nicht-majoritären Institutionen und internationalen Organisationen kommt es zu einem kosmopolitischen Bias: Politische Eliten innerhalb dieser Institutionen sind besonders kosmopolitisch eingestellt, wohingegen die kommunitaristische Position in nationalen Parlamenten und Parteien tendenziell verbreiteter ist (vgl. Zürn 2018: 12). In diesem Zuge lässt sich eine gesunkene Responsivität des politischen Systems gegenüber den Präferenzen der stärker kommunitaristisch eingestellten Gesellschaftsschichten feststellen (vgl. Elsässer et al. 2017), was bei diesen Menschen zu dem Gefühl führt, nicht ausreichend von den nicht-majoritären Institutionen repräsentiert zu werden.

Autoritäre Populist ${ }^{\star}$ innen bedienen die Gefühle der politischen Exklusion und der Machtlosigkeit dieser (meist kommunitaristisch eingestellten) Bevölkerungsschichten über das Narrativ der „schweigenden
Mehrheit" und der "korrupten Eliten“ (Zürn 2017: 4f.). Insbesondere im Nachgang zu Krisensituationen ist dieses Narrativ vielversprechend, da sich in diesen Momenten der kosmopolitische Bias der Politiken der nicht-majoritären Institutionen zeigt, wie im Fall der Eurokrise oder der sogenannten Flüchtlingskrise (vgl. ebd.: 30).

\section{Methodik}

Vor dem Hintergrund dieser drei wesentlichen Erklärungsansätze für das Aufkommen des autoritären Populismus wird im Folgenden der AfD-Diskurs zum Thema Klimaschutz inhaltsanalytisch ausgewertet. Die Datengrundlage der Inhaltsanalyse bilden die Pressemitteilungen des AfDBundesvorstands zum Thema „Klima“ aus dem Jahr 2019. Pressemitteilungen sind das zentrale Medium der Pressearbeit von Parteien. Kommunikationswissenschaftliche Untersuchungen zeigen, dass die Übernahmequoten von Pressemitteilungen von Parteien hoch sind und Themen und Timing der Medienberichterstattung beeinflussen (vgl. Schulz 2011: 294). Pressemitteilungen haben als organisationsseitig autorisiertes Vermittlungsmedium zum Ziel, die offizielle Position der Bundespartei und ihrer führenden Vertreter ${ }^{*}$ innen zu aktuellen Themen und Ereignissen zu kommunizieren. Pressemitteilungen richten sich neben den Journalist ${ }^{*}$ innen als 
Zwischenvermittler*innen an die eigentliche Zielgruppe der Informationsangebote (vgl. Szyszka/Christoph 2015: 801), in diesem Fall alle Wählerinnen und potenziellen Unterstützer*innen der Partei. Sie stellen also ein geeignetes Medium dar, um sowohl die aktuelle inhaltliche Positionierung der Partei als auch die kommunikative Strategie und Adressierung der (potenziellen) Wählerschaft zu untersuchen.

Ein besonderer Fokus wird aus verschiedenen Gründen auf den Zeitraum von September bis Dezember 2019 gelegt. Zum einen wurden in diesen Monaten vermehrt Pressemitteilungen zum Thema Klima durch die AfD veröffentlicht. Dies deckt sich zeitlich mit der Ankündigung des AfD-Parteivorsitzenden Gauland, der Ende September forderte, den Kampf gegen den Klimaschutz zur neuen Hauptaufgabe seiner Partei zu machen (Kamann 2019). Zum anderen fanden in diesen Monaten mit dem globalen Klimastreik und den Beratungen des Klimakabinetts über das Klimaschutzpaket am 20. September sowie der UN-Klimakonferenz und der Ankündigung des europäischen Green Deals Anfang Dezember wichtige Ereignisse im Zusammenhang mit der Klimapolitik statt.

Die kategoriengeleitete qualitativ-orientierte Textanalyse nach Mayring/Fenzel (2014) ist für diese Fragestellung eine geeignete Methode, da mit ihr einerseits vergleichsweise große Datenmengen ausgewertet werden können und sie aufgrund ihres regelgeleiteten Vorgehens intersubjektiv überprüfbar ist, sowie andererseits durch die qualitativ-interpretative Analyse auch latente Sinngehalte der untersuchten Texte erfassen und diese kontextualisieren kann (vgl. ebd.: 543). Das Vorgehen stellt eine Kombination aus deduktiver Kategorienanwendung und induktiver Kategorienbildung dar.

Vor dem Hintergrund der theoretischen Überlegungen aus Kapitel 2 wird die Analyse durch die drei deduktiven Oberkategorien strukturiert: 1. Ökonomische Unsicherheit; 2. kultureller Wertewandel und 3. politische Entfremdung. Auch wenn die drei Erklärungen, wie oben angemerkt, in Wechselwirkung zueinander stehen und keine monolithischen Ansätze darstellen, eignen sich diese Dimensionen dennoch aus zwei Gründen zur Ableitung idealtypischer Kategorien: Zum einen wird die wissenschaftliche Debatte entscheidend durch diese drei Erklärungsansätze strukturiert und die meisten Beiträge lassen sich einer der Strömungen zuordnen. Zum anderen verweisen die drei Erklärungen auf im Kern klar voneinander unterscheidbare Ursachen für das Erstarken des autoritären Populismus, auch wenn diese in manchen Kontexten zusammenhängen oder sich potenzieren mögen (vgl. Kraemer 2018: 288). Gleichzeitig identifizieren sie damit unterschiedliche Argumentationsweisen 
und Narrative, die von autoritär-populistischen Akteur*innen aufgegriffen und bedient werden.

Die drei Oberkategorien wurden im Zuge der Auswertung mit induktiv aus dem Quellenmaterial entwickelten Kategorien präzisiert. In Bezug auf das Abstraktionsniveau wurden alle Argumente und Stellungnahmen im Material zu den jeweiligen klimapolitischen Themen und Fragestellungen in induktive Kategorien codiert. In diesem Fall sind diese induktiven Kategorien gleichbedeutend mit sogenannten Frames.

Frames oder Rahmen werden hier mit Rein und Schön verstanden als „way of selecting, organizing, interpreting, and making sense of a complex reality“ (1993: 146). Frames führen ihnen zufolge zu verschiedenen Sichtweisen auf die Welt und erzeugen damit eine Vielzahl sozialer Realitäten. Das Framing eines bestimmten Sachverhalts kann verschiedene Bewertungen und damit zusammenhängend unterschiedlichen Handlungen als Antwort darauf zur Folge haben. Politische Akteur ${ }^{*}$ innen greifen daher im „Kampf um Ideen“ (Stone 2002: 13) auf bestimmte Frames als strategisches Mittel zurück, um einen Sachverhalt zu erfassen und in einer bestimmten Weise zu konstruieren - mit dem Ziel, Deutungshoheit zu erlangen. Den Frames liegen dabei immer bestimmte Werte, Normen und Ideen, ein "appreciative system“, zugrunde (ebd.: 146f.). Die Überlegungen der Frame-Analyse eignen sich für die Fragestellung dieser Arbeit als Erweiterung der Inhaltsanalyse, da sie zu verstehen hilft, inwiefern die AfD bestimmte klimapolitische Sachverhalte unter Bezug auf "appreciative systems" diskursiv rahmt, um populistisches Protestpotenzial zu aktivieren.

Angelehnt an das Verfahren der kategoriengeleiteten qualitativ-orientierten Textanalyse nach Mayring und Fenzel (2014) wurde bei der Inhaltsanalyse, die mithilfe der Open Access-Webapplikation QCAmap durchgeführt wurde, in den folgenden Schritten vorgegangen: Als Kodiereinheit als kleinstem auszuwertenden Materialbestandteil wurden mehrere Wörter mit Sinnzusammenhang, bedeutungstragende Phrasen festgesetzt. Die Kontexteinheit, der größte in eine Kategorie fallende Textbestandteil, besteht in einem Absatz, also einem in mehreren Sätzen entfalteten Argument. Die Auswertungseinheit stellt die Summe aller Pressemitteilungen dar.

In einem ersten Test-Durchlauf von etwa einem Drittel aller Pressemitteilungen wurden induktive Kategorien innerhalb der deduktiven Oberkategorien aus dem Text entwickelt. Nach dem ersten Durchlauf wurden Kategorien, welche sich auf einen ähnlichen Gegenstand bezogen, zusammengefasst und reduziert. Aufgrund mehrfacher Quernennungen von 
Ausprägungen aller Oberkategorien wurde eine weitere Oberkategorie „Klimahysterie“ entwickelt. Die darin enthaltenen Frames "Vernunft statt Ideologie“ und „Sozialismus-Vorwurf“ lassen sich keiner der drei Oberkategorien klar zuordnen, sondern bilden vielmehr die Grundlage der drei Kategorien, da in ihnen die ablehnende Haltung der AfD zu Klimaschutzpolitik im Allgemeinen zum Ausdruck kommt. Im endgültigen Materialdurchlauf erfolgte dann anhand des induktiv entwickelten Kategoriensystems mit den vier deduktiven Oberkategorien die Kodierung aller 29 Pressemitteilungen.

\section{Der AfD-Klimadiskurs}

Die Grundlage für die drei Oberkategorien bildet die Sonder-Kategorie „Klimahysterie“, die in den drei Frames enthalten ist, mithilfe derer die AfD den Klimaschutz als ideologisch getriebenes Projekt darstellt und den Klimawandel als solchen - mehr oder weniger direkt - leugnet. Da diese Frames nicht inhaltlich argumentieren, sondern den Klimawandel an sich und die Notwendigkeit des Klimaschutzes infrage stellen und sich in keine der drei Oberkategorien einordnen lassen, werden sie an dieser Stelle der Vollständigkeit halber nur kurz skizziert.

In einer Pressemitteilung zur Rede der AfD-Fraktionsvorsitzenden Alice Weidel während der Generaldebatte über den Haushalt des Bundeskanzleramts im September 2019 wird wiedergegeben, wie sie die Schwere des Klimawandels leugnet und dessen Ausmaße als reine Einbildung darstellt: Weidel sagt, die Bundesregierung verschwende „Abermilliarden, um imaginierte Weltuntergänge in ferner $\mathrm{Zu}$ kunft abzuwenden“ (Weidel 11.09.2019). An anderer Stelle wird der Einfluss des Menschen auf das Klima von AfD-Umweltpolitiker Karsten Hilse infrage gestellt und der Klimawandel als ein „natürlicher Prozess seit Hunderten Millionen von Jahren" beschrieben (AfD 19.09.2019).

In diesem Sinne wird Klimaschutzpolitik als rein ideologisches und vernunftbefreites Projekt geframed: Die Energiewende wird als „unproduktive[s] ideologische[s] Prestigeprojekt[]" beschrieben (Weidel 16.10.2019) und Klimaschutzpolitik als „Klimahysterie“ (Brandner 18.12.2019), „Klimawahn“ (Padzderski 19.09.2019) oder ,ideologiepolitischer Irrweg“ (Weidel 18.10.2019) bezeichnet. An mancher Stelle werden Klimaschutzmaßnahmen der Bundesregierung oder der Europäischen Kommission als "grün-sozialistische Ideologie“ (Weidel 11.09.2019) geframed und damit an das kollektive Gedächtnis der Deutschen und die negativen Erfahrungen mit dem „real existierenden Sozialismus“ im Sinne eines „appreciative system“ appelliert. 


\section{1 Ökonomische Unsicherheit}

Innerhalb des Themenkomplexes Klimaund Umweltpolitik thematisiert die AfD in ihren Pressemitteilungen besonders häufig die Energiewende. Alle Aussagen, die auf die (vermeintlichen) finanziellen Folgen für die Bürger ${ }^{*}$ innen abzielen und die Kosten von Energiewende und Klimaschutz in den Vordergrund rücken, werden unter dem Frame „Bürger*innen zahlen Klimaschutz" (A2) zusammengefasst. Die AfD stellt die Energiewende als sich in hohen Stromkosten bemerkbar mache (Weidel 05.09.2019). Aus diesem Grund fordert die AfD, „die Energiewende, die außerdem zum Verlust von Millionen von Arbeitsplätzen führen wird, sofort zu beenden“ (Padzderski 19.09.2019). Dabei wird mehrfach behauptet, dass die ,verkorkste Energiewende“ und der „überhastete Kohleausstieg“ die Bürger*innen „immer tiefer in die Tasche greifen“ lasse und die Stromversorgung in Gefahr (A3) sei (Gauland 13.03.2019). Durch den Verweis auf eine drohende Gefährdung der Versorgungssicherheit mit Strom in Deutschland werden Ängste vor strukturellen Veränderungen adressiert und das Projekt der Energiewende diskreditiert.

Neben der Energiewende werden sämtliche Klimaschutzmaßnahmen stets als zusätzliche Kosten für „die ganz normalen Leute“ gerahmt (Weidel 27.09.2019). Die

AfD unterstellt, die Bundesregierung beute „unter dem Vorwand des ,Klimaschutzes $[$ [...] die Steuerbürger skrupellos aus" und nutze die „Klima-Hysterie“, um Steuererhöhungen durchzusetzen (Weidel 18.10.2019). Die AfD stellt fest, dass eine Verteuerung des Autofahrens und der Energienutzung "vor allem die kleinen Leute“ treffe. Aus dieser Kritik an der ungerechten Verteilung von Kosten durch Klimaschutzmaßnahmen leitet sie die pauschale Folge ab: „Es gibt keine ,sozial gerechte Klimapolitik.“(ebd.)

Die eigentlichen Ziele und der Sinn der Klimaschutzmaßnahmen, nämlich die Reduktion von Treibhausgasen und die damit verbundene Einhaltung von Klimazielen, werden in den Pressemitteilungen nicht thematisiert. Vielmehr werden diese Maßnahmen als ein Selbstzweck oder gar als absichtliche Drangsalierung der Bevölkerung („Klima-Abzocke“; Weidel 27.09.2019) dargestellt.

Indem die AfD stets betont, dass sämtliche Klimaschutzmaßnahmen zu Lasten der „kleinen Leute“, „des kleinen Mannes“, der „ganz normalen Leute“ oder der „Normalbürger" gingen, adressiert sie explizit die Bevölkerungsschichten, die, wie oben festgestellt, in den letzten beiden Jahrzehnten Lohnstagnation oder -verluste erlebt haben. Sie appelliert an die Abstiegsängste, die soziale Verunsicherung und das Gefühl des „Ausgeliefertsein“ der Menschen, die, 
wie Kohlrausch (2018) zeigt, ein wesentlicher Treiber der Entscheidung sind, AfD zu wählen.

Diese Ängste versucht die AfD zu schüren, indem sie Klimaschutz als Gefährdung von Wohlstand und Arbeitsplätzen (A1) rahmt. Mit ihrer „absurden Klimapolitik“ trage die Bundesregierung „entscheidend zur weiteren Verarmung der Mittelschicht bei" und treibe Wirtschaft und Arbeitsmarkt „noch tiefer in die Krise“, sagt AfD-Fraktionsvorsitzende Alice Weidel (27.09.2019). Damit knüpft sie an das Gefühl vieler Menschen an, gesellschaftlichen Abstieg zu erfahren und von dem Wirtschaftswachstum der letzten Jahre nicht zu profitieren.

Sehr häufig wird die Gefährdung des Wirtschaftsstandorts Deutschland und der deutschen Automobilindustrie durch Klimaschutzmaßnahmen betont. Die AfD appelliert damit an das ,appreciative system" des in Deutschland tief verankerten Stolzes auf die Automobilindustrie als Arbeitsplatzgarant und Motor für Wohlstand und Wirtschaftswachstum: Der „pseudo-
Klimaschutz" sei „ein Programm zur Industrie- und Arbeitsplatzvernichtung" (Weidel 11.09.2019) und der „Klimaaktionismus der Bundesregierung" nichts anderes als „eine dramatische Wettbewerbsverzerrung für unsere heimische Wirtschaft" (Protschka 20.09.2019). ,Wirtschaftssadistische Ökoideologen" gefährdeten Wachstum in Deutschland und die „über Jahrzehnte hinweg mühsam aufgebaute Automobilindustrie und Zuliefererbetriebe" würden durch „meinungsprägende Ökopopulisten“ für deren „vorgeblich grünes Gewissen“ zerlegt (Meuthen 22.01.2019). Die „Automobilindustrie als Motor der Industrie“ werde „mutwillig im Namen des Klimawahns beschädigt" (Weidel 16.10.2019) und tausende Arbeitsplätze durch die ,industriefeindliche Grünen-Politik der KlimaAktivisten“vernichtet (Kalbitz 06.08.2019).

Neben der Sorge vor gesellschaftlichem Abstieg adressiert die AfD mit diesen Horrorszenarien die Angst vieler Menschen, insbesondere der traditionellen Arbeiter ${ }^{\star}$ innenschaft, vor einem (durch die Globalisierung bedingten) Strukturwandel. Es wird versucht, die ökonomischen aufzubringen, indem dieser für Ungerechtigkeiten und möglicherweise drohende ökonomische Verluste verantwortlich gemacht wird. 
Globalisierungsverlierer ${ }^{\star}$ innen gegen Klimaschutz aufzubringen, indem dieser für Ungerechtigkeiten und möglicherweise drohende ökonomische Verluste verantwortlich gemacht wird. Besonders deutlich wird dies bei einer Pressemitteilung im Namen von Alexander Gauland, in der der AfD-Fraktionsvorsitzende insinuiert, „dass nun tausende Kohlekumpel vom Ruhrpott bis in die Lausitz keine Zukunft mehr haben und von Armut bedroht sind“, sei „den Grünen egal“ (Gauland 15.09.2019). Hier werden die berechtigten Zukunftssorgen der alten Industriearbeiter ${ }^{*}$ innenschaft instrumentalisiert, um die Notwendigkeit des Klimaschutzes infrage zu stellen. Die an dieser Stelle vorgenommene Gegenüberstellung der Interessen von Menschen in ländlichen Industrieregionen und den politischen Forderungen der (meist als kosmopolitisch assoziierten) Grünen leitet zur nächsten Oberkategorie über.

\subsection{Kultureller Wertewandel}

In mehreren Pressemitteilungen der Bundespartei kontrastiert die AfD die "normalen Leute" mit dem Feindbild einer "großstädtischen Elite“. Indem sie auf Unterschiede zwischen den Werten und Lebensweisen dieser beiden Gruppen verweist, zielt sie explizit auf die neue gesellschaftliche Konfliktlinie zwischen (städtischen) Kosmopolit*innen und Kommunitaristen ${ }^{\star}$ innen (A5) ab. Als Feindbild und Inbegriff kosmopolitischer Werte und Lebensweisen fungieren hierbei Mitglieder und Anhänger*innen der Partei Bündnis 90/Die Grünen. Indem AfD-Parteivize Georg Padzderski behauptet, „die Grünen schwimmen im linksliberalen Mainstream der Elite und üben die kulturelle Hegemonie aus" (Padzderski 11.06.2019), zielt er auf den mit dem kulturellen Liberalismus verbundenen Wertewandel (,silent revolution“) ab und befeuert den oben beschriebenen kulturellen 'Backlash' der eher traditionalistisch eingestellten Bevölkerung.

Unter Verweis auf die globale Konkurrenz stellt Padzderski fest, dass über Deutschlands Zukunft „keine CO2-Steuer, keine Biotonne, kein Radfahrweg und kein Verbot von Fleisch" entscheide (ebd.). Klimaschutz wird hierdurch reduziert auf die Verteuerung von Konsum, als ein Angriff auf die persönliche Freiheit und als kultureller Imperativ im Konflikt mit traditionellen Lebensweisen. Im Zusammenhang mit der Debatte um eine CO2-Steuer betont die AfD stets die Auswirkungen auf Berufspendler*innen, also insbesondere Bewohner*innen ländlicher Regionen, von Kleinstädten und Vororten, und adressiert damit die „alte Mittelklasse“, die sich in ihrer Lebensweise und Werten von der kosmopolitischen und oftmals urbanen "neuen Mittelklasse“ unterscheidet (vgl. Reckwitz 2017: 274ff.). Den Gegensatz zwischen einer moralisierenden und „abgehobenen Elite“ und den „einfachen 
Leuten" (A6) betont Gauland in einer Stellungnahme zum Grünen-Parteitag 2019, indem er deren Beschlüsse als „eine Mischung von Schizophrenie und moralischer Überheblichkeit, die alle zulasten der Bürger gehen" beschreibt (Gauland 18.11.2019). Der „moralisierende grüne Zeigefinger" werde überall schmerzhaft zu spüren sein, unter anderem dadurch, dass sich Autofahren und Fleischkonsum verteuere (ebd.). Diese Warnung vor der Verteuerung einer bestimmten - meist eher als kommunitaristisch gelesenen Lebensweise illustriert beispielhaft, dass kulturelle und ökonomische Erklärungen miteinander verschränkt sein können und von autoritär-populistischen Akteur*innen diskursiv in ein und demselben Argument verarbeitet werden.

Anstelle des Klimaschutzes versucht die AfD in zahlreichen Pressemitteilungen den Umweltschutz positiv zu besetzen und als Alternative ins Spiel zu bringen (A8). Das „appreciative system“, das diesem Frame zugrunde liegt, ist die semantisch eng mit dem Begriff der Heimat verbundene Umwelt. So plädiert die AfD für „eine Umweltpolitik, die nicht alleine der Klimaindustrie dient, sondern unsere Heimat, ihre Menschen und ihre Natur schützt“"(Padzderski 19.09.2019). Die eigens dafür gestartete Kampagne „Grüne stoppen - Umwelt schützen!“ mit eigener Kampagnen-Website verfolgt das Ziel, (grüne) Klimaschutzpolitik als interessengesteuerte „Klimaindustrie“ zu rahmen und den Schutz der ",heimatlichen Umwelt" dieser entgegenzustellen. Der erneuerbare Energieträger der Windkraftanlagen steht hier als Inbegriff für diesen konstruierten Gegensatz zwischen Klima und Umwelt, indem behauptet wird, dass durch Windkraftanlagen in Deutschland bis zu 100.000 Vögel und bis zu 200.000 Fledermäuse pro Jahr stürben und „Lebensraum für Fauna und Flora und Kulturlandschaften in unvorstellbarer Größe vernichtet" würde (ebd.). Die AfD macht hier also eine andere Abwägung auf: Die (sichtbar und lokal) sterbenden Vögel aufgrund von Windkraftanlagen sind ihnen wichtiger als (global) sterbende Tierarten aufgrund des Klimawandels.

Diese Betonung des Nationalen und der Heimat wird im Sinne der kulturellen Erklärung als Abwehrreaktion auf eine sich verändernde, globalisierte Welt verstanden. Das Narrativ der zu schützenden Heimat adressiert die sogenannten „Somewheres“, die oftmals aufgrund geringerer Bildung und finanzieller Ausstattung stärker an lokale oder regionale Strukturen gebunden sind (vgl. Goodhart 2020). Durch die prognostizierte Vernichtung der heimatlichen Umwelt sollen die Ängste derjenigen geschürt werden, die Veränderung ohnehin eher ablehnen. 


\subsection{Politische Entfremdung}

In diesem Zusammenhang werden die sogenannten „Anywheres“ als Feindbild konstruiert. Tendenziell eher kosmopolitisch eingestellte Entscheidungsträger*innen werden als „E-Auto-Planungsbürokraten in Brüssel und Berlin" dargestellt (Weidel 13.12.2019). Dieses Framing der Entscheidungsträger ${ }^{\star}$ innen suggeriert, dass sich Politiker*innen in Deutschland und der EU der demokratischen Kontrolle entzogen hätten („Planungsbürokraten“) und in den Zentren der Macht (insb. Brüssel und Berlin) über das Leben der Mehrheit entscheiden und dabei Partikularinteressen (hier: E-Auto) verfolgen würden.

Heftige Kritik wird an den nicht-majoritären Organisationen (A7) in der EU geübt. In einer Pressemitteilung zum Green Deal, der im Dezember 2019 von der der EU-Kommission beschlossenen wurde, nennt AfD-Bundesvorstandsmitglied Sylvia Limmer diesen Maßnahmenplan für eine nachhaltigere EU-Wirtschaft den "größte[n] und schwerwiegendste[n] Angriff auf die freie Gesellschaft in Europa seit dem Fall der Berliner Mauer" (Limmer 13.12.2019). Damit versucht Limmer, die Klimaschutzpolitik der EU als undemokratische Maßnahme darzustellen und Assoziationen mit Freiheitsberaubungen in der DDR hervorzurufen. Der Green Deal der EU-Kommission, der unter anderem durch Investitionen in regenerative
Energieträger und eine höhere Bepreisung fossiler Energieträger die EU-Mitgliedsstaaten bis 2050 klimaneutral machen will, sei „ein Sammelsurium an totalitären Maßnahmen, die ein Diktator nicht hätte toppen können" (ebd.). Dadurch wird versucht, das Gefühl der Menschen, nicht ausreichend von den nicht-majoritären Institutionen repräsentiert zu werden, zu nutzen, um die EU-Klimaschutzpolitik als undemokratisch darzustellen.

Limmer beklagt in diesem Zusammenhang die „eigenmächtige Kompetenzerweiterung durch die Kommission“ und unterstellt, dass ,die Kommission sogar in den Haushalt der Nationalstaaten einzugreifen und Steuern umzulenken" gedenke (ebd.), was weder in dem Green Deal der EU-Kommission vorgesehen noch in den EU-Verträgen rechtlich geregelt ist. Die politische Erklärung führt an, dass die gesunkene Responsivität des politischen Systems gegenüber den Präferenzen der stärker kommunitaristisch eingestellten Gesellschaftsschichten eine der Ursachen für den Aufstieg des autoritären Populismus ist (vgl. Zürn 2018: 12). Dieses Gefühl der Machtlosigkeit und politischen Exklusion wird hier instrumentalisiert, um den Green Deal zu delegitimieren.

Dasselbe Gefühl der Machtlosigkeit bedient auch das Narrativ der stillen Mehrheit (A9), das sich die AfD zu eigen macht, wenn Parteivorsitzender Jörg Meuthen in 
einer Pressemitteilung zum Green Deal beklagt, dass die EU-Bürger`innen ,immer größere Summen an Steuern für ihre eigene Entmündigung, Entrechtung und Enteignung" zahlten (Meuthen 11.12.2019). Meuthen stellt hier die Bevölkerung der EU-Mitgliedsstaaten als stille Mehrheit dar, die die „ökosozialistische Umgestaltung unseres Kontinents" zu bezahlen hätte und keinerlei Einfluss auf politische Entscheidungen nehmen könne. In diesem Sinne ruft Limmer zum Widerstand auf: „Wenn wir als Bürger nicht geschlossen dagegen vorgehen, wird dies das Ende der freien Gesellschaft sein, wie wir sie kennen." (Limmer 13.12.2019)

Während die AfD einerseits das koordinierte Vorgehen der EU ablehnt, bezweifelt sie im Zusammenhang mit dem Klimapaket der Bundesregierung, „dass dieser nationale ,Klimaschutz-Alleingang der Bundesregierung in einer global vernetzten Welt auch nur die kleinste Auswirkung auf das Weltklima haben wird“ (Protschka 20.09.2019). Die Begrenztheit der deutschen Klimaschutzmaßnahmen und die Kontrastierung des nationalen Handelns mit dem globalen Klimawandel (A7) nutzt die AfD an mehreren Stellen, um die Maßnahmen an sich infrage zu stellen. Auch hier bedient sie das Gefühl der Machtlosigkeit in einer globalisierten Welt, um im selben Atemzug die eigenen nationalen Interessen über gemeinsame globale Interessen zu stellen.

\subsection{Quantitative Auswertung: Häufigkeit der Frames}

Die quantitative Auswertung der Häufigkeit des Auftretens der Kategorien zeigt, dass die Frames innerhalb der Oberkategorie „ökonomische Unsicherheit" mit insgesamt 35 Kodierungen deutlich häufiger auftreten als die Frames innerhalb der Oberkategorie "politische Entfremdung" (14) und „kultureller Wertewandel“ (12). Die Frames innerhalb der zusätzlichen Oberkategorie „Klimahysterie“ kommen mit 24 Kodierungen ebenfalls häufig vor (siehe Abbildung 1).

Die Positionierung der AfD zu klimapolitischen Fragen rückt damit besonders häufig Argumente in den Vordergrund, die sich innerhalb der ökonomischen Erklärung zum Erstarken des autoritären Populismus verorten lassen. Der abschließende Kodierungsdurchlauf hat gezeigt, dass sich die meisten Argumente klar einer der Kategorien innerhalb der Oberkategorien zuordnen lassen. Wenn innerhalb eines Satzes verschiedene (Ober-)Kategorien auftauchten, wurde dies durch eine Mehrfachzuordnung dieser Textbestandteile berücksichtigt.

Die Auswertung der innerhalb der Oberkategorien induktiv entwickelten Kategorien zeigt, dass der Frame „Gefährdung von Wohlstand und Arbeitsplätzen" besonders häufig auftritt (17), gefolgt von dem Frame „Bürger ${ }^{*}$ innen zahlen Klimaschutz“ (15). Der Frame „Nicht-majoritäre 


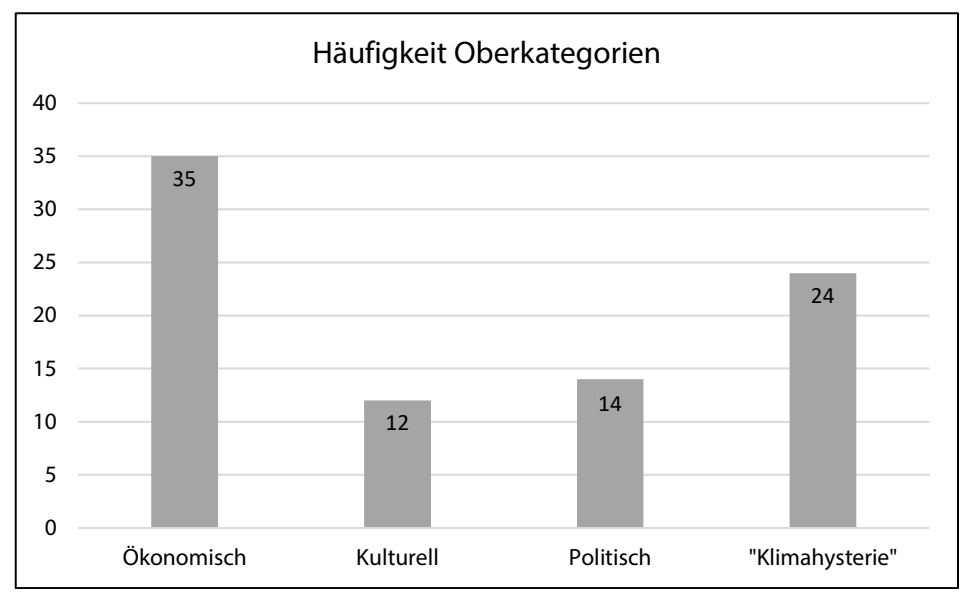

Abbildung 1: Häufigkeit des Auftretens der deduktiven Oberkategorien

\begin{tabular}{|l|l|l|}
\hline Oberkategorie 1: Ökonomische Unsicherheit \\
\hline Code & Kategorienname & Häufigkeit \\
\hline A1 & Gefährdung von Wohlstand und Arbeitsplätzen & 17 \\
A2 & Bürger*innen zahlen Klimaschutz & 15 \\
A3 & Stromversorgung in Gefahr & 3 \\
\hline
\end{tabular}

\begin{tabular}{|l|l|l|}
\hline \multicolumn{2}{|l|}{ Oberkategorie 2: Kultureller Wertewandel } & Häufigkeit \\
\hline Code & Kategorienname & 5 \\
\hline A4 & Umwelt statt Klima & 5 \\
A5 & Kosmopolit*innen vs. Kommunitarist*innen & 2 \\
A6 & Elite vs. einfache Leute & \\
\hline
\end{tabular}

\begin{tabular}{|l|l|l|}
\hline \multicolumn{2}{|l|}{ Oberkategorie 3: Politische Entfremdung } & Häufigkeit \\
\hline Code & Kategorienname & 8 \\
\hline A7 & Nicht-majoritäre Organisationen & 4 \\
A8 & National vs. Global & 2 \\
A9 & Stille Mehrheiten & \\
\hline
\end{tabular}

\begin{tabular}{|l|l|l|}
\hline \multicolumn{2}{|l|}{ Oberkategorie 4: „Klimahysterie“ } & Häufigkeit \\
\hline Code & Kategorienname & 16 \\
A10 & Vernunft statt Ideologie & 6 \\
A11 & Klimawandel-Leugnung & 2 \\
A12 & Sozialismus-Vorwurf & \\
\hline
\end{tabular}

Tabellen 1 bis 4: Häufigkeit der Frames innerhalb der Oberkategorien 
Organisationen" innerhalb der politischen Oberkategorie kommt ebenfalls vergleichsweise häufig vor (8). Die beiden kulturellen Frames „Umwelt statt Klima“ und „Kosmopolit ${ }^{\star}$ innen vs. Kommunitarist ${ }^{\star} i n n e n^{\text {“ }}$ (je 5) werden in den Pressemitteilungen etwa so oft aufgegriffen wie der politische Frame „National vs. Global“. Etwas weniger oft sind die Frames „Stromversorgung in Gefahr" (3) sowie „Elite vs. einfache Leute“ und „Stille Mehrheiten“ (je 2) vertreten.

\section{Fazit und Diskussion}

Dieser Beitrag hatte zum Ziel, zu untersuchen, inwiefern die AfD mit ihrem Klimadiskurs populistisches Protestpotenzial adressiert. Dafür wurde unter Bezug auf verschiedene aktuelle Arbeiten aus der Populismusforschung, der Politischen Soziologie und der Politischen Theorie ein theoretisches Framework entwickelt. Die drei deduktiven Oberkategorien der ökonomischen Unsicherheit, des kulturellen Wertewandels und der politischen Entfremdung strukturierten die induktive Kategorienbildung der Inhaltsanalyse.

Die quantitative Auswertung der Häufigkeit des Auftretens der Kategorien zeigt, dass die Frames innerhalb der Oberkategorie „ökonomische Unsicherheit" etwa dreimal häufiger verwendet werden als die Frames der beiden anderen Oberkategorien, die etwa gleich häufig vorkommen. Die
Betonung ökonomischer Auswirkungen des Klimaschutzes auf die Bevölkerung steht im Zentrum der Positionierung der AfD. Den Kern dieser Argumentation bilden Horrorszenarien, die die Gefährdung des Wirtschaftsstandorts Deutschland und der Autoindustrie sowie horrende zusätzliche (Strom-)Kosten für die „einfachen Leute“, Autofahrer*innen und Pendler*innen prognostizieren. In ihren Stellungnahmen zum Thema Klima adressiert die AfD besonders häufig die Angst vor ökonomischen Einbußen und soziale Abstiegsängste, die in Deutschland insbesondere in den unteren Gesellschaftsschichten, aber auch darüber hinaus weitverbreitet sind (vgl. Kohlrausch 2018: 5). Steigende Ungleichheit, Lohnstagnation und -verluste sowie eine zunehmende Prekarisierung bilden die Grundlage dafür, dass das AfD-Narrativ vom Klimaschutz als milliardenschweres Arbeitsplatzund Industrievernichtungsprogramm, das von den „einfachen Leuten “ bezahlt werden müsse, auf fruchtbaren Boden fällt.

Der AfD-Klimadiskurs zielt darüber hinaus auch auf die neue gesellschaftliche Konfliktlinie zwischen Kosmopolit*innen und Kommunitarist ${ }^{\star}$ innen ab, die von der kulturellen und der politischen Erklärung ins Zentrum der Analyse gerückt wird. Klimaschutzmaßnahmen werden von der AfD als Angriff auf traditionelle Lebensweisen und als großstädtisches Elitenprojekt gerahmt. Als Feindbild dient insbesondere die Partei der Grünen. Der Schutz der positiv konnotierten 
Wie können Klimaschutzmaßnahmen sozial gerecht ausgestaltet werden, um die Angriffsfläche des ökonomisch motivierten populistischen Protests zu verringern?

und eng mit dem Begriff der Heimat verbundenen (lokalen) Umwelt wird dem (globalen) Klimaschutz vorgezogen. Damit adressiert die AfD eher lokal gebundene „Somewheres“, denen mehr am Schutz der heimatlichen Umwelt als an globalen Maßnahmen zum Schutz des Weltklimas liegt.

In diesem Zusammenhang werden die Klimaschutzmaßnahmen nicht-majoritärer Organisationen wie der EU-Kommission als undemokratisch und als Eingriff in die nationalstaatliche Souveränität gerahmt. Damit zielt die AfD explizit auf das Gefühl der politischen Exklusion und Machtlosigkeit eher kommunitaristisch eingestellter Bevölkerungsschichten. Der Frame der schweigenden Mehrheit illustriert exemplarisch, wie das von der politischen Erklärung ausgemachte populistische Protestpotenzial adressiert wird.

Vor dem Hintergrund der vielen Anknüpfungspunkte für einen populistischen
Diskurs überrascht es nicht, dass die AfD dieses Thema zur neuen Hauptaufgabe der Partei ausgewählt hat. Der Klimaschutz und der damit verbundene Strukturwandel sind mit einschneidenden Veränderungen verbunden. Daher bietet sich das Thema an, um an die Ängste der Bevölkerung vor sozialem Abstieg und Veränderung zu appellieren. In diesem Politikfeld fallen ökonomische, kulturelle und politische Konflikte zusammen und werden von der AfD über verschiedene Frames adressiert. Die Untersuchung hat gezeigt, dass allen drei Ansätze der Populismusforschung zumindest in Bezug auf Klimaschutz und das Framing der AfD als autoritär-populistischer Partei in Bezug auf dieses das Politikfeld - Erklärungskraft innewohnt. Durch die Berücksichtigung jeder der drei Erklärungsmuster wurden die verschiedenen Dimensionen des AfD-Klimadiskurses und dessen Vielschichtigkeit sichtbar.

Im Sinne der eingangs angesprochenen demokratisierenden Funktion populistischen Protests als Ausdrucksform marginalisierter Interessen (vgl. Laclau 2018; Mouffe 2018) lassen sich aus diesen Ergebnissen drei zentrale Herausforderungen für eine Klimaschutzpolitik ableiten, die verschiedene Interessen berücksichtigt und in möglichst hohem Maße Akzeptanz in der Bevölkerung erfährt: Wie können Klimaschutzmaßnahmen sozial gerecht ausgestaltet werden, um die Angriffsfläche des ökonomisch motivierten 
populistischen Protests zu verringern? Eine Klimaschutzpolitik, die die Lasten gerecht verteilt und im Sinne der Gemeinwohlorientierung eine nachhaltige Infrastruktur und sozialen Ausgleich zur Bewältigung des erforderlichen Strukturwandels schafft, könnte dem zentralen Gegenargument der Klimaschutzgegner ${ }^{*}$ innen die Luft aus den Segeln nehmen. Die Gelbwesten-Bewegung in Frankreich als Reaktion auf die höhere Besteuerung fossiler Kraftstoffe sind ein Paradebeispiel dafür, welche Folgen eine Klimaschutzpolitik haben kann, unter der insbesondere ohnehin schon prekär lebende Bevölkerungsschichten zu leiden haben (vgl. Wahl/Aguiton 2019).

Wie kann Klimaschutz kommuniziert werden, ohne moralisierend zu wirken? Wer darauf hofft, durch den Appell an das grüne Gewissen der Menschen und individuelle Konsumentscheidungen anstelle kollektiver und struktureller Maßnahmen dem Klimawandel beizukommen, missachtet die Funktionslogiken des auf Wachstum basierenden kapitalistischen Wirtschaftssystems. Werden zudem aus einer privilegierten Position heraus bestimmte Lebensweisen und Konsumverhalten unterer Schichten moralisch stigmatisiert, kann dies eine sinkende Akzeptanz und eine Abwehrhaltung gegen Klimaschutz zur Folge haben, wie die heftigen Reaktionen auf das Umweltsau-Lied Ende 2019 vortrefflich illustrieren.
Wie kann die demokratische Debatte über die Ausgestaltung des Klimaschutzes gestärkt und Transparenz hergestellt werden? Auch wenn das Ergreifen von Klimaschutzmaßnahmen unumgänglich ist, braucht es eine Diskussion über die Ausgestaltung dieser. Aufklärung und klare Kommunikation über die Notwendigkeit einer global koordinierten Bekämpfung des Klimawandels könnten zudem zur Akzeptanz gegenüber Klimaschutzmaßnahmen beitragen. Um den Vorwurf der undemokratischen Entscheidungsfindung nachhaltig zu schwächen, bräuchte es darüber hinaus institutionelle Reformen, um die Demokratiedefizite in der EU zu verringern.

\section{LITERATUR}

Alvaredo, Facundo/Chancel, Lucas/Piketty, Thomas/Saez, Emmanuel/Zucman, Gabriel/Freundl, Hans/Gebauer, Stephan (2018). Die Weltweite Ungleichheit: Der World Inequality Report 2018. München: C.H. Beck.

Beck, Ulrich (1986): Risikogesellschaft: Auf dem Weg in eine andere Moderne. Frankfurt am Main: Suhrkamp.

Biskamp, Floris (2019): Ökonomie ist kulturell, Kultur ist ökonomisch: Einspruch zur politischen Ökonomie des Populismus. In: PROKLA. Zeitschrift für Kritische Sozialwissenschaft Jg. 49/3, S. 463-476.

Bonikowski, Bart (2017): Three Lessons of Contemporary Populism in Europe and the United States. In: The Brown Journal of World Affairs Jg. 23, S. 9-24.

de Wilde, Pieter/Koopmans, Ruud/Merkel, Wolfgang/ Strijbis, Oliver/Zürn, Michael (Hrsg.) (2019): The Struggle over Borders. Cosmopolitanism and Communitarianism. Cambridge: Cambridge University Press. 
Dörre, Klaus/Bose, Sophie/Lütten, John/Köster, Jakob (2018): Arbeiterbewegung von rechts? Motive und Grenzen einer imaginären Revolte. In: Berliner Journal Für Soziologie Jg. 28/1, S. 55-89.

Elsässer, Lea/Hause, Svenja/Schäfer, Armin (2017): „Dem Deutschen Volke“? Die ungleiche Responsivität des Bundestags. In: Zeitschrift für Politikwissenschaft Jg. 27/2, S. 161-180.

Freeden, Michael (1998): Is Nationalism a Distinct Ideology? In: Political Studies Jg. 46/4, S. 748-765.

Goodhart, David (2020): The Road to Somewhere: Wie wir Arbeit, Familie und Gesellschaft neu denken müssen. München: millemari.

Hambauer, Verena/Mays, Anja (2018): Wer wählt die AfD? - Ein Vergleich der Sozialstruktur, politischen Einstellungen und Einstellungen zu Flüchtlingen zwischen AfD-WählerInnen und der WählerInnen der anderen Parteien. In: Zeitschrift Für Vergleichende Politikwissenschaft Jg. 12/1, S. 133-154.

Häusler, Alexander (2019): Kumulative Radikalisierung: Der völkisch-autoritäre Populismus der AfD. In: Forschungsjournal Soziale Bewegungen Jg. 32/1, S. 83-88.

Inglehart, Ronald Franklin/Norris, Pippa (2016): Trump, Brexit and the Rise of Populism: Economic Have-Nots and Cultural Backlash. In: Faculty Research Working Paper Series Jg. 16-026, Cambridge: Harvard Kennedy School. Online verfügbar unter https://www.hks.harvard.edu/publications/trump-brexit-and-rise-populism-economic-have-nots-and-cultural-backlash (06.09.2020).

Jagers, Jan/Walgrave, Stefan (2007): Populism as political communication style: An empirical study of political parties' discourse in Belgium. In: European Journal of Political Research Jg. 46/3, S. 319-345.

Kamann, Matthias (2019): Die AfD und die „sogenannte Klimaschutzpolitik“, In: „Die Welt“, 29.09.2019. Online verfügbar unter https://www.welt.de/politik/deutschland/ article201093000/CO2-Emissionen-Die-AfD-und-die-sogenannte-Klimaschutzpolitik.html (30.04.2020).

Keller, Reiner (2008): Wissenssoziologische Diskursanalyse: Grundlegung eines Forschungsprogramms. Wiesbaden: Springer VS.
Kohlrausch, Bettina (2018): Abstiegsängste in Deutschland. In: Working Paper Forschungsförderung, 58, Düsseldorf: Hans-Böckler-Stiftung. Online verfügbar unter https://www.boeckler.de/pdf/p fofoe WP 0582018. pdf (06.09.2020).

Korte, Karl-Rudolf/Leggewie, Claus/Lewandowsky, Marcel (2015): Partei am Scheideweg: Die Alternative der AfD. In: Blätter für deutsche und internationale Politik, S. 59-67. Online verfügbar unter https://www.blaetter.de/ ausgabe/2015/juni/partei-am-scheideweg-die-alternative-der-afd (06.09.2020).

Kraemer, Klaus (2018): Sehnsucht nach dem nationalen Container. Zur symbolischen Ökonomie des neuen Nationalismus in Europa. In: Leviathan Jg. 46/2, S. 280-302.

Laclau, Ernesto (2018): On Populist Reason. London: Verso.

Manow, Philip (2018): Die politische Ökonomie des Populismus. Berlin: Suhrkamp.

Manow, Philip (2020): (Ent-)Demokratisierung der Demokratie. Berlin: Suhrkamp.

Mayring, Philipp/Fenzel, Thomas (2014): Qualitative Inhaltsanalyse. In: Baur, Nina/Blasius, Jörg (Hrsg.): Handbuch Methoden der empirischen Sozialforschung. Wiesbaden: Springer VS, S. 543-556.

Milanović, Branko (2016): Global Inequality: A New Approach for the Age of Globalization. Cambridge: Cambridge University Press.

Mouffe, Chantal (2018): Für einen linken Populismus. Berlin: Suhrkamp.

Mudde, Cas (2004): The Populist Zeitgeist. In: Government and Opposition Jg. 39/4, S. 542-563.

Nachtwey, Oliver (2016): Die Abstiegsgesellschaft. Berlin: Suhrkamp.

Nachtwey, Oliver (2019): Vorwort. In: Chibber, Vivek: Kapitalismus verstehen. Das ABC des Kapitalismus. Berlin: Brumaire, S. 1-5.

Radtke, Jörg/Canzler, Weert/Schreurs, Miranda A./ Wurster, Stefan (Hrsg.) (2019): Energiewende in Zeiten des Populismus. Wiesbaden: Springer VS. 
Reckwitz, Andreas (2017): Die Gesellschaft der Singularitäten. Berlin: Suhrkamp.

Rein, Martin/Schön, Donald (1993): Reframing Policy Discourse. In: Fischer, Frank/Forester, John (Hrsg.): The argumentative turn in policy analysis and planning. London: UCL Press, S. 145-166.

Schulz, Winfried (2011): Politische Kommunikation. Wiesbaden: Springer VS.

Stone, Deborah (2012): Policy paradox: The art of political decision making. New York: Norton.

Szyszka, Peter/Christoph, Cathrin (2015): Medienarbeit (Presse-/Medienarbeit). In: Fröhlich, Romy/Szyska, Peter/ Bentele, Günter (Hrsg.): Handbuch der Public Relations. Wiesbaden: Springer VS, S. 795-813.

Wahl, Peter/Aguiton, Christoph (2019): Gilets Jaunes: Anatomie Einer Ungewöhnlichen Sozialen Bewegung. Köln: PapyRossa.

Zürn, Michael (2018): Autoritärer Populismus vs. offene Gesellschaft - eine neue Konfliktlinie? In: Böll.brief Demokratie und Gesellschaft. Online verfügbar unter https://www.boell.de/sites/default/files/boll.brief 7 autoritarer populismus vs. offene gesellschaft.pdf?dimension $1=$ division demo $(06.09 .2020)$.

Zürn, Michael (2017): How Non-Majoritarian Institutions Make Silent Majorities Vocal: A Political Explanation of Authoritarian Populism. Unveröffentlichtes Working Paper aus drei Workshops, WZB Berlin Social Science Center and Freie Universität Berlin.

\section{DATENKORPUS AFD-PRESSEMITTEILUNGEN}

Alternative für Deutschland (AfD) (19.09.2019). AfD startet Kampagne: Grüne stoppen - Umwelt schützen! Online verfügbar unter https://www.afd.de/afd-startet-kampagne-gruene-stoppen-umwelt-schuetzen/ (30.04.2020).

Brandner, Stephan (18.12.2019): Die CO2-Klimahysterie ist kein guter Ratgeber - die Zeche zahlt der Bürger. Online verfügbar unter https://www.afd.de/stephan-brandner-dieco2-klimahysterie-ist-kein-guter-ratgeber-die-zeche-zahltder-buerger/ (30.04.2020).

Gauland, Alexander (15.02.2019): Die GRÜNEN sabotieren eine vernünftige deutsche Politik. Online verfügbar unter https://www.afd.de/alexander-gauland-die-gruenen-sabotieren-eine-vernuenftige-deutsche-politik/ (30.04.2020).

Gauland, Alexander (13.03.2019): Energiewende - Die Klimahysterie bedroht unseren Wohlstand. Online verfügbar unter https://www.afd.de/alexander-gauland-energiewende-die-klimahysterie-bedroht-unseren-wohlstand/ (30.04.2020).

Gauland, Alexander (18.11.2019). Die Politik der GRÜNEN geht zulasten der Bürger. Online verfügbar unter https://www.afd.de/alexander-gauland-die-politik-der-gruenen-geht-zulasten-der-buerger/ (30.04.2020).

Gottschalk, Kay (19.07.2019): Die Bahnfahrt muss billiger werden und nicht das Fliegen teurer! Online verfügbar unter https://www.afd.de/kay-gottschalk-die-bahnfahrt-mussbilliger-werden-und-nicht-das-fliegen-teurer/(30.04.2020).

Kalbitz, Andreas (06.08.2019): Frank Bsirskes klimastreikende Gewerkschafter verraten Arbeitnehmer. Online verfügbar unter https://www.afd.de/andreas-kalbitz-frank-bsirskes-klimastreikende-gewerkschafter-verraten-arbeitnehmer/ (30.04.2020).

Limmer, Sylvia (13.12.2019): Der „Green Deal“ ist ein massiver Angriff auf die freie Gesellschaft! Online verfügbar unter https://www.afd.de/sylvia-limmer-der-greendeal-ist-ein-massiver-angriff-auf-die-freie-gesellschaft/ (30.04.2020).

Meuthen, Jörg (22.01.2019): Wirtschaftssadistische Ökoideologen gefährden Wachstum in Deutschland. Online verfügbar unter https://www.afd.de/ joerg-meuthen-zur-iwf-prognose-\%E2\%88\%92-wirtschaftssadistische-oekoideologen-gefaehrden-wachstum-in-deutschland/ (30.04.2020).

Meuthen, Jörg (10.09.2019): Jörg Meuthen wirft Grünen Panikmache vor. Online verfügbar unter https://www. afd.de/joerg-meuthen-gruene-wollen-neue-schuldenfuers-weltklima-machen-nicht-mit-der-afd/ (30.04.2020).

Meuthen, Jörg (13.05.2019): GRÜNE wollen neue Schulden fürs ,Weltklima' machen - Nicht mit der AfD! Online verfügbar unter https://www.afd.de/klimadebatte-joerg-meuthen-wirft-gruenen-panikmache-vor/ (30.04.2020).

Meuthen, Jörg (28.11.2019): Klimanotstand - EU wird für Klimaflüchtlinge unzumutbar! Online verfügbar unter https://www.afd.de/joerg-meuthen-klimanotstand-eu-wird-fuer-klimafluechtlinge-unzumutbar/ (30.04.2020). 
Meuthen, Jörg (11.12.2019): Green Deal ist plumper Aktionismus aus politischen Gründen. Online verfügbar unter https://www.afd.de/joerg-meuthen-green-deal-ist-plumper-aktionismus-aus-politischen-gruenden/ (30.04.2020).

Padzderski, Georg (11.06.2019): Streitschrift „Die Grünen endlich angreifen, statt sie hochzureden“. Online verfügbar unter https://www.afd.de/georg-pazderski-streitschrift-die-gruenen-endlich-angreifen-statt-sie-hochzureden/ (30.04.2020).

Padzderski, Georg (19.09.2019): Deutscher Klima-Wahn ist ein ignoranter Alleingang. Online verfügbar unter https://www.afd.de/georg-pazderski-deutscher-klima-wahn-ist-ein-ignoranter-alleingang/ (30.04.2020).

Padzderski, Georg (07.10.2019): „Extinction Rebellion“-Extremisten nötigen Berliner. Online verfügbar unter https://www.afd.de/georg-pazderski-extinction-rebellion-extremisten-noetigen-berliner/ (30.04.2020).

Protschka, Stephan (20.09.2019): Klimapaket der Bundesregierung treibt Höfesterben weiter voran. Online verfügbar unter https://www.afd.de/stephan-protschka-klimapaket-der-bundesregierung-treibt-hoefesterben-weiter-voran/ (30.04.2020).

Protschka, Stephan (01.10.2019): Niederländische Landwirte protestieren zu Recht gegen Klimawandel-Vorwürfe. Online verfügbar unter https://www.afd.de/ stephan-protschka-niederlaendische-landwirte-protestieren-zu-recht-gegen-klimawandel-vorwuerfe/ (30.04.2020).

von Storch, Beatrix (27.11.2019): Für Meinungs- und Wissenschaftsfreiheit an deutschen Unis ist es fünf nach zwölf. Online verfügbar unter https://www.afd.de/beatrixvon-storch-fuer-meinungs-und-wissenschaftsfreiheit-andeutschen-unis-ist-es-fuenf-nach-zwoelf/ (30.04.2020).

Weidel, Alice (05.09.2019): Die Energiepolitik der Bundesregierung wird zum Albtraum für die Bürger. Online verfügbar unter https://www.afd.de/alice-weidel-die-energiepolitik-der-bundesregierung-wird-zum-albtraum-fuer-die-buerger/ (30.04.2020).

Weidel, Alice (11.09.2019): Der pseudo-Klimaschutz ist ein Programm zur Industrie- und Arbeitsplatzvernichtung. Online verfügbar unter https://www.afd.de/alice-weidel-der-pseudo-klimaschutz-ist-ein-programm-zur-industrie-und-arbeitsplatzvernichtung/ (30.04.2020).

Weidel, Alice (27.09.2019): Klimapaket Groko - Kfz-Steuer wird sich mehr als verdoppeln. Online verfügbar unter https://www.afd.de/alice-weidel-klimapaket-groko-kfzsteuer-wird-sich-mehr-als-verdoppeln/ (30.04.2020).

Weidel, Alice (16.10.2019): Fehlgeleitete Wirtschaftspolitik macht Deutschland zum Abstiegskandidaten. Online verfügbar unter https://www.afd.de/alice-weidel-fehlgeleitete-wirtschaftspolitik-macht-deutschland-zum-abstiegskandidaten/ (30.04.2020).

Weidel, Alice (18.10.2019): Die Klimapolitik der Bundesregierung geht immer zu Lasten der kleinen Leute. Online verfügbar unter https://www.afd.de/alice-weidel-die-klimapolitik-der-bundesregierung-geht-immer-zu-lasten-der-kleinen-leute/ (30.04.2020).

Weidel, Alice (04.12.2019): Stromausfall - per Regierungsblackout in die Katastrophe. Online verfügbar unter https://www.afd.de/alice-weidel-stromausfall-per-regierungsblackout-in-die-katastrophe/ (30.04.2020).

Weidel, Alice (13.12.2019): China düpiert die Elektroauto-Planwirtschaft in Deutschland und der EU. Online verfügbar unter https://www.afd.de/alice-weidel-chinaduepiert-die-elektroauto-planwirtschaft-in-deutschlandund-der-eu/ (30.04.2020).

\section{ZUM AUTOR}

Georg Sturm, 23, studiert im Master Internationale Beziehungen an der Freien Universität Berlin, der Humboldt-Universität zu Berlin und der Universität Potsdam. Seine Studienschwerpunkte sind Populismusforschung, Lateinamerikastudien und politische Theorie. Derzeit arbeitet er als freier Autor für die Tageszeitung (taz) und das Neue Deutschland (nd).

An dem Beitrag haben folgende Redaktionsmitglieder im Review, Betreuung und Lektorat mitgearbeitet: Cathrin Mund, Andreas Schulz, Hendrik Erz und Tanja Strukelj. 
Perspektive 


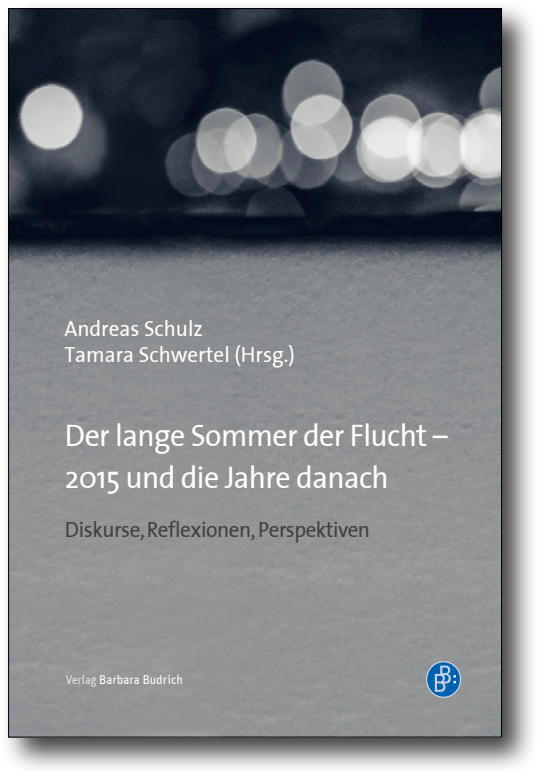

Was bleibt fünf Jahre nach dem Sommer 2015 und den anhaltenden Fluchtbewegungen? Welche Bedeutung kommt den Ereignissen von 2015 immer noch zu und in welche Lebensbereiche ragen diese hinein? Der vorliegende Sammelband nimmt sich dem Thema Flucht aus verschiedenen Perspektiven an. Er vereint dabei Beiträge von Nachwuchswissenschaftler*innen aus der Soziologie, Sozialpsychologie, Kulturund Sozialanthropologie sowie der Kommunikations- und Kulturwissenschaft und gibt Anstöße für die andauernden Debatten um das Thema Flucht nach Europa. Der Sammelband ist ein Projekt des soziologiemagazin e.V.
Andreas Schulz

Tamara Schwertel (Hrsg.)

\section{Der lange Sommer der Flucht - 2015 und die Jahre danach}

\author{
Diskurse, Reflexionen, Perspektiven
}

2020. 306 Seiten. Kart.

$39,00 €(D), 40,10 €(A)$

ISBN 978-3-8474-2467-3

Auch als eBook: 978-3-8474-1611-1

Der Verein setzt sich für die Sichtbarkeit nachwuchswissenschaftlicher Perspektiven ein und bringt im gleichnamigen Heft diese zu unterschiedlichen Themen der Gesellschaft zur Sprache. Auch auf dem Blog unter www.soziologiemagazin.de werden sozialwissenschaftliche Auseinandersetzungen gefördert.

Die Herausgeber*innen:

Andreas Schulz, MA,

Soziologie, Kultur- und Sozialanthropologie, Publizistik- und Kommunikationswissenschaft (Leipzig, Bern und Wien)

Tamara Schwertel, MA, Soziologie, Politikwissenschaft und Philosophie (Frankfurt am Main, Paris und Lyon) 


\title{
Heterotopie Ilmpark
}

Raum zur Suche nach einer neuen Umweltästhetik

von Mats Werchohlad

\begin{abstract}
Der Park an der Ilm in Weimar bietet für die hier behandelten Themen im Spannungsfeld von Umwelt und Gesellschaft einen besonderen Raum. Einerseits spiegeln und verdichten sich darin vielfältige gesellschaftliche wie ökologische Entwicklungen und Phänomene, andererseits enthält er das Potential, um darin gemeinschaftlich nach neuen Lösungsansätzen zu suchen. Um dieses Potential zur Entfaltung zu bringen, werden im Rahmen dieser Abhandlung die Eckpunkte und Argumentationslinien einer seit drei Jahren anhaltenden Auseinandersetzung meinerseits mit diesem Ort nachgezeichnet. Aus einer heterotopologischen Betrachtung sollen dabei neue Aspekte und Deutungsansätze für die Zeit der Weimarer Klassik und des Historischen Staatlichen Bauhaus zu Tage gefördert werden. Die Auseinandersetzung zielt darauf ab, den Park in seinen heterotopischen Eigenschaften wieder stärker hervortreten zu lassen und hierdurch abschließend als Ausgangspunkt für die Suche nach einer neuen Umweltästhetik zu konstituieren.
\end{abstract}

\section{Schlagwörter}

Klimawandel; Umweltästhetik; Heterotopie; Ilmpark; Bauhaus 


\section{Einleitung}

Zunehmend werden in historischen Parkanlagen Auswirkungen des Klimawandels sichtbar (vgl. Rohde/Krellig 2004). Zuletzt haben die coronabedingten Ausgangs- und Kontaktbeschränkungen außerdem zu einer beträchtlichen Mehr- und Übernutzung städtischer und historischer Parkanlagen geführt (vgl. Kropmanns 2020; Kurianowicz 2020). In diesem Sinne spiegeln und verdichten sich aktuelle ökologische und gesellschaftliche Entwicklunbesonderer Weise an diesen Orten.

Der Park an der Ilm in Weimar ist hierfür ein herausragendes Beispiel: Hier ist vor allem der wertvolle historische Baumbestand durch die anhaltende Trockenheit massiv

angegriffen und gefährdet. Unvorhersehbar werfen alte Bäume große Äste ab und führen damit zu einer Beeinträchtigung der Verkehrssicherheit. Zahlreiche Bäume mussten daher gefällt werden (vgl. Grau 2018). Pflanzen und Wiesen leiden, Wege und Uferbereiche werden durch starke Regenfälle unter- oder abgespült. Unbegehbare Wege, Baustellen und unsichere Bereiche führen immer häufiger zur Sperrung ganzer Parkpassagen (vgl. Grau et al. 2018). Ein sich veränderndes Nutzungsverhalten, neue Ansprüche und daraus resultierende Interessenskonflikte haben zuletzt zu einer erheblichen Vermüllung, Auseinandersetzungen und Vandalismus geführt und stellen die verantwortliche Klassik Stiftung mit der Bewirtschaftung des Parks vor substantielle Herausforderungen (vgl. Weber 2020).

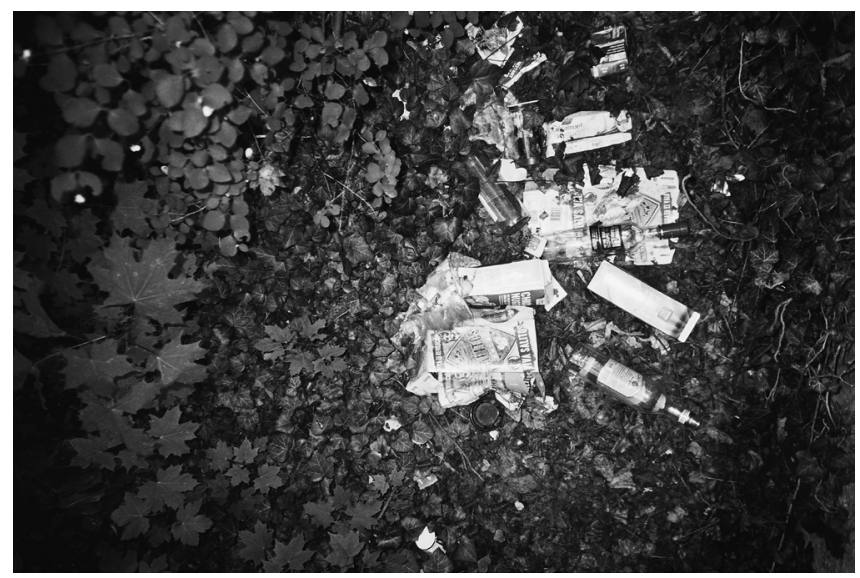

Abbildung 1: Der Park an der Ilm hinter dem russischen Ehrenfriedhof: Detailansicht (Foto: Mats Werchohlad) 
Die notwendige Neuausrichtung und Anpassung des Parkkonzepts sowie eine für 2021 geplante Bundesgartenschau in Erfurt werden von Seiten der Stiftung daher zum Anlass genommen, um mit einem Themenjahr unter dem Titel „Neue Natur“ einen zukunftsgerichteten Diskurs im Spannungsfeld Mensch - Umwelt - Natur anzustoßen. Der Park an der Ilm bietet hierfür einen spannenden räumlich-historischen Bezugspunkt: Als gartenkünstlerische Manifestation der Weimarer Klassik und als solche Teil des UNESCO-Weltkulturerbes steht der Park sinnbildlich für eine historische Idealkonzeption von Natur und ihrer Gestaltung (vgl. Müller-Wolf 2007), welche angesichts der aktuellen Entwicklungen jedoch immer weiter in Diskrepanz zur Wirklichkeit zu rücken scheint. Gleichzeitig sieht der Naturphilosoph Gernot Böhme in Johann Wolfgang von Goethes Naturverständnis, das in der Theorie des englischen Landschaftsgartens wurzelt, einen paradigmatischen Anknüpfungspunkt für die Suche nach einer neuen Naturästhetik (vgl. Böhme 1989).

Der Park bietet insofern das Potential, im Zusammenspiel der Weimarer Institutionen, zum Forschungs-, Erlebnis- und Verhandlungsraum für die kollektive Suche nach einer neuen Naturästhetik zu werden. Um dieses Potential jedoch vollständig entfalten zu können, so lautet die These und der Ansatz der vorliegenden Abhandlung, sollte dieser Ort (erneut) eine Reihe von spezifischen Eigenschaften und Funktionen aufweisen, die sich durch Michel Foucaults Konzept der Heterotopie beziehungsweise der heterotopologischen Wissenschaft (Foucault 2013) besonders gut beschreiben und neu implementieren ließen.

Diesem Ziel, den Park in seinen heterotopischen Eigenschaften (wieder stärker) hervortreten zu lassen, widmet sich eine seit etwa dreieinhalb Jahren, in verschiedenen Formen und Formaten laufende, wissenschaftliche und diskursive Auseinandersetzung mit diesem Ort und seiner Geschichte. Ausgangspunkt hierfür war ein Beitrag in einem 2017 durchgeführten Masterprojekt im Studienfach Urbanistik an der Bauhaus-Universität Weimar (Welch Guerra 2018) und eine daran anknüpfende Abschlussthesis (Werchohlad 2018). Inhaltlich wurde dabei dem räumlichen $\mathrm{Zu}$ sammenhang des Parks und der Entwicklungsgeschichte des historischen Bauhauses in Weimar nachgegangen. Zum Einsatz kamen dabei vielfältige, fachübergreifende Methoden und Ansätze. Ergänzt und weiterentwickelt wurden die Ergebnisse durch öffentliche Spaziergangs- und Gesprächsformate im Rahmen des Weimarer Kunstfestes 2019 und die Durchführung einer interdisziplinären Vortrags- und Seminarreihe an der Bauhaus-Universität Weimar. Die im Verlauf dieses Prozesses gesammelten Erfahrungen, Erkenntnisse und Ergebnisse bilden die Grundlage für die vorliegenden Ausführungen. 
Trotz, dass es sich bei der Annäherung in diesem Beitrag somit um keine dezidiert soziologische Herangehensweise handelt, eröffnen sich über den räumlichen Ausgangspunkt eine Vielzahl inhaltlicher Anknüpfungspunkte für das hier verhandelte Thema. Dieser Ansatz könnte für die soziologische Betrachtung in diesem Sinne einen ergänzenden Mehrwert bieten. Denn gerade die Auseinandersetzung mit Raum und den gesellschaftlichen Konfigurationen spezifischer Räume stand in der klassischen Soziologie lange Zeit im durch die Arbeiten Henri Lefebvres und Michel Foucaults sowie die unter dem Begriff Spatial Turn populär gewordene Deutung des Geographen und Stadtforschers Edward Soja (vgl. Günzel/Kümmerling 2010: 90) ist eine übergreifende, auf den Raum zielende Wende in den Sozialwissenschaften zu verzeichnen (vgl. Soja 1989; 2009: 249). Dennoch konstatiert der Soziologe Markus Schroer: „Noch immer tut sich die Soziologie schwer mit dem Raum“ (2006: 9). Anhand von Foucaults Konzept der Heterotopie soll dieser Beitrag dazu einladen, im Rahmen des Themenzusammenhangs dieses Magazins im und über den Park an der Ilm in einen fachübergreifenden Austausch zu kommen.

\section{Die Heterotopien}

Es gibt gleichfalls - und das wohl in jeder Kultur, in jeder Zivilisation wirkliche Orte, wirksame Orte, die in die Einrichtung der Gesellschaft hineingezeichnet sind, sozusagen Gegenplatzierungen oder Widerlager, tatsächlich realisierte Utopien, in denen die wirklichen Plätze innerhalb der Kultur gleichzeitig repräsentiert, bestritten und gewendet sind, gewissermaßen Orte außerhalb aller Orte, wiewohl sie tatsächlich geortet werden können. Weil diese Orte ganz andere sind als alle Plätze, die sie reflektieren oder von denen sie sprechen, nenne ich sie im Gegensatz zu den Utopien die Heterotopien. (Foucault 1992: 39)

Der Begriff der Heterotopie wurde vom Philosophen Michel Foucault in einem Radiovortrag aus dem Jahr 1966 entwickelt. Demnach schaffen Heterotopien durch Ordnungen oder Illusionen utopische, jedoch gleichsam reale Räume, die alle anderen Räume in Frage stellen können (vgl. Foucault 2013: 19). Zur Betrachtung und Analyse dieser Orte skizziert Foucault (vgl. 2013: 11) eine heterotopische Wissenschaft, die er als Heterotopologie bezeichnet. Diese Ausführungen wurden noch einmal für einen Vortrag unter dem Titel „Andere Räume" (Foucault 1992) überarbeitet. In sechs Grundsätzen versucht er darin, seine Vorstellung dieser in sich widersprüchli- 
chen und produktiven Räume (vgl. Ruoff 2013: 192) näher zu umreißen. Zunächst setzt Foucault (vgl. 1992: 40) voraus, dass alle Gesellschaften Heterotopien hervorbringen. Zweitens merkt er an, dass die Funktionen eines heterotopischen Ortes in Bezug auf zeitliche und gesellschaftliche Veränderungen von wechselhaftem Charakter sein können (vgl. Foucault 1992: 41). Als wesentliches Merkmal beschreibt er drittens die Fähigkeit dieser Orte, vielfältige Räume zu integrieren und in sich zu vereinen (vgl. Foucault 1992: 42f.). Viertens werden diese heterotopischen Eigenschaften vor allem wirksam, „wenn die Menschen mit ihrer herkömmlichen Zeit brechen" (Foucault 1992: 43). Ihre Zeitlichkeit - die Akkumulation verschiedener Zeiten in Form eines Archives oder umgekehrt die Flüchtigkeit eines Festes konstituieren diesbezüglich verschiedene Typen der Heterotopie. In Beziehung und Abgrenzung zu den umliegenden Räumen und Strukturen steht diese dabei für ein spezielles System der Öffnung und Isolation (vgl. Foucault 1992: 44). Im letzten Punkt seiner Ausführungen verweist Foucault noch einmal auf die besondere Eigenschaft, gegenüber den wirklichen, etablierten Strukturen als Illusions- oder Kompensationsraum zu fungieren (vgl. 1992: 45). Als Beispiele für verschiedene Arten von Heterotopien werden unter anderem Schiffe, Freudenhäuser, Friedhöfe und speziell Gärten angeführt (vgl. Foucault 1992: 42). In einem weiteren
Radiovortrag komplementierte Foucault seine Ausführungen zu Heterotopien. Unter dem Titel „Der utopische Körper“ (Foucault 2013) beschreibt er die Sehnsucht nach heterotopischen Orten. Diesen ursprünglichen Drang bezieht Foucault vor allem auf die menschliche Körperlichkeit und das Unvermögen, sich von dieser zu befreien (vgl. 2013: 36). Ein zentrales Element stellen diesbezüglich Spiegel und Spiegelillusionen dar.

Mit diesem Set an Eigenschaften, das Foucault zur Beschreibung von Heterotopien vorgibt, lassen sich somit spezifische Räume untersuchen und in den Blick nehmen. In den Fokus treten dabei ihre räumlichen Funktionen und Qualitäten für die Gesellschaft. Da sich diese in und mit ihrer Umwelt in ständigem Wandel befinden, soll nachfolgend nicht so sehr der Frage nachgegangen werden $o b$, sondern vielmehr wann und wie der Park in seinen heterotopischen Eigenschaften hervorgetreten ist.

\section{Krisenhafte Zeiten - Der Park während der Weimarer Klassik}

Welche neuen Aspekte eröffnet die heterotopologische Betrachtung des Parks in Bezug auf die Weimarer Klassik?

Kaum etwas in den romantisch und klassisch angelegten Partien des Parks 
an der Ilm offenbart heute Anzeichen der als krisenhaft empfundenen Phase seiner Entstehung. Wird der Park jedoch als Heterotopie begriffen, eröffnet sich in seiner lieblich arrangierten gärtnerischen Gestaltung und seinen - dem Anschein nach - natürlich gewachsenen Naturszenen ein Kompensationsraum, „der so vollkommen, so sorgfältig, so wohlgeordnet ist wie der unsrige ungeordnet, mißraten und wirr ist" (Foucault 1992: 45). Der Park lässt sich in diesem Sinne als kompensatorischer Gegenraum deuten, denn der „Holzbedarf von Glasmanufakturen, häufige Kriege und die Verschwendungssucht der herrschenden Dynastie hatten die Thüringer Wälder, die wesentliche Ressource des Landes, devastiert" (Grober 2010: 121). Aus diesem Grund wurden unter der Regentschaft Anna Amalias, die ab 1758 die Weimarer Regierungsgeschäfte für ihren noch minderjährigen Sohn übernahm, mitten im Siebenjährigen Krieg, die überhaupt ersten auf Nachhaltigkeit ausgerichteten Forstreformen umgesetzt (vgl. Grober 2010: 121). Gleichzeitig gewannen durch ihren auf Kultur- und Naturnähe zielenden Regierungsstil die in den Wäldern und Gärten gelegenen Orte und Sommerresidenzen außerhalb der Schloss- und Stadtmauern an Bedeutung (vgl. Busch-Salmen et al. 1998). Damit einher ging eine Zusammenführung der einzelnen Park- und Landschaftspartien rund um das Schloss (vgl. Huschke 1951: 52). Katalysiert wurden diese Tendenzen durch ein weiteres krisenhaftes Ereignis am 6. Mai 1774: den Brand des Weimarer Stadtschlosses (vgl. Huschke 1951: 52). Durch diesen Umstand fand die Weimarer Hofgesellschaft, samt ihres prominenten Neuzugangs Johann Wolfgang von Goethe, im Park einen Rückzugsort, an dem sich die Rousseausche Sehnsucht nach dem Zurück zur Natur (vgl. Warning 2015: 184) in den vielfältigen kleinen Salons und Festorten, Gartentheatern und Naturszenen in neuen Formen kultivieren ließ (vgl. Eckardt et al. 2009: 52). Den aufklärerischen Idealen eines standesübergreifenden Gemeinschaftsgeistes folgend, wird die Niederreißung der den Park umgebenden Zäune und Mauern als beinahe performativer und sinnbildlicher Akt beschrieben (vgl. Huschke 1955: 28). Mit dem Bau des Römischen Hauses finden sowohl der herzogliche Wunsch nach Zurückgezogenheit und Naturnähe als auch ein auf Repräsentation und Gesellschaft zielender Ort mitten in der zugänglichen Parköffentlichkeit eine Vereinigung (vgl. Berger 2001: 32). Durch seine neue Dimension steht das Römische Haus stellvertretend für einen Bruch in der Parkgestaltung: Von kleinmaßstäblichen romantischen Szenerien zu einer großmaßstäblichen, an der Klassik und den Gegebenheiten des Ortes orientierten Landschaftsgestaltung (vgl. Müller 2001: 109). Mit dem Baubeginn nur zwei Jahre nach dem prägenden Ereignis der Französischen Revolution lässt sich das Römische Haus, wie auch die neue 
Maßstäblichkeit in der Parkgestaltung, als räumliche Ausdrucksform für den Versuch interpretieren, ,,in neue Dimensionen von Kunst, Kultur und Wissenschaft vorzustoßen, um die Fähigkeit zu erlangen, die historische Herausforderung der Revolution zu ,gewältigen“" (Müller 2007: 157). Im Giebelfeld des Hauses findet sich dieser neue Anspruch versinnbildlicht, indem Allegorien für Kunst, Wissenschaft, Musik und Theater mit Obst- und Ackerbau zur universellen Vereinigung gelangen (vgl. Beyer 2001: 142).

Diesen Anspruch verfolgte auch Goethe in seinen Werken, die zu einem großen Teil in seinem Gartenhaus im Park verfasst wurden. In Goethes Roman „Die Wahlverwandtschaften" (Goethe 1809) finden diese Vereinigung und Grenzauflösung zwischen wirklicher Welt und Illusion, Wissenschaft und Kunst, Liebe und Vernunft, Zweifel und Wahnsinn im Spiegel gemeinschaftlicher Landschaftsgartengestaltung ihre Vereinigung und literarische Entsprechung (vgl. Haberl 2012). Abschließend ergänzt und erweitert Goethe damit beinahe exemplarisch den heterotopischen Raum des Parks. Denn nach Michel Foucault ist der Garten als konkreter Raum

seit der frühsten Antike ein Ort der Utopie. Wenn man den Eindruck hat, Romane ließen sich leicht in Gärten ansiedeln, so liegt das daran, dass der Roman zweifellos aus der Institution der Gärten entstanden ist. Das Schreiben von Romanen ist eine gärtnerische Tätigkeit. (2013: 15)

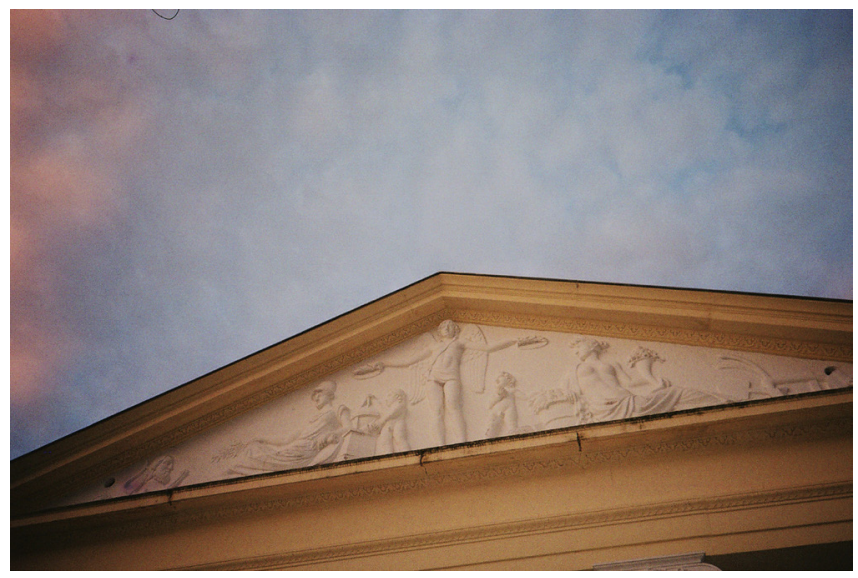

Abbildung 2: Das Giebelrelief von Johann Peter Kaufmann am Römischen Haus zeigt die Vereinigung von Kunst, Wissenschaft, Obst- und Ackerbau (Foto: Mats Werchohlad) 
Aus den krisenhaften Determinanten eröffnet sich im Park ein grenzauflösender, archivarischer, illusorischer, kompensatorischer und bühnenhafter Raum, der alle wesentlichen Kriterien und Eigenschaften des Heterotopiekonzepts aufweist. In seinen heterotopischen Eigenschaften tritt der Park an der Ilm somit als Spiegelund Bewältigungsraum der damaligen Strömungen und Krisenereignisse im Spannungsfeld von Natur, Individuum und Gesellschaft hervor. Der Park offenbart emotionale, wissenschaftliche und künstlerische Gestaltungsansätze, um der Komplexität der als krisenhaft wahrgenommenen Zeit eine wieder als natürlich empfundene, neu geordnete Wirklichkeit entgegenzusetzen.

\section{Der Park als Abbild einer roman- tisch verklärten Gegenwart}

Inwieweit steht eine hegemoniale Zuschreibung dieses Ortes seinen heterotopischen Potentialen entgegen?

Wer heute durch den Park spaziert, findet sich in einem idealisierten und vollkommen harmonischen Abbild jener krisenhaften Zeit wieder. Der Park an der Ilm, der zwischen den Landschaftsgärten der Schlösser Tiefurt und Belvedere eingebettet liegt, erscheint, trotz der ihn umgebenden urbanen Räume, als ein vollkommen und natürlich erhaltener Kosmos aus dem 18.
99

In seinen heterotopischen Eigenschaften tritt der Park an der Ilm somit als Spiegel- und Bewältigungsraum der damaligen Strömungen und Krisenereignisse im Spannungsfeld von Natur, Individuum und Gesellschaft hervor.

Jahrhundert. Als gartenkünstlerische Manifestation und räumliches Erbe der Weimarer Klassik ist der Park an der Ilm in dieser Qualität seit 1998 Teil des UNESCO-Weltkulturerbes (vgl. UNESCO 1998). Dass der Park in seiner heutigen, beinahe unveränderten Form in dieser Weise erhalten geblieben ist, lässt sich auf vielfältige Gründe zurückführen. Hervorzuheben ist hierfür eine beinahe kultisch betriebene Verehrung Goethes, die in der Weimarer Stadtgeschichte, im Erhalt, der Pflege und der Auseinandersetzung mit diesem Erbe eine besondere Kontinuität aufweist (vgl. Merseburger 1999). Die Mythisierung und damit einhergehende Musealisierung der Wirkstätten Goethes wurden, wie die Aufstellung seiner Büsten in der Anna Amalia Bibliothek zeigt (vgl. Knoche 2007: 237), schon zu seinen 
Lebzeiten betrieben (vgl. Müller 2007: 158). Gleiches lässt sich seit dem Tod des Herzogs Carl August und spätestens mit Goethes Tod 1832 für den Erhalt und die Nutzung der Gebäude sowie die landschafts- und denkmalpflegerischen Erwägungen im Park an der Ilm nachvollziehen (vgl. Huschke 1955: 117). Immer wieder setzten sich die herzoglichen Nachfahren, wie beispielsweise Großherzog Carl Alexander, auch gegen Vorschläge, wie die des renommierten Landschaftsarchitekten Fürst Pückler, zur Wehr, um den Park im Abbild dieser Zeit zu bewahren (Huschke 1951: 161). Jüngst durchgeführte Baumaßnahmen, wie die Rekonstruktion der romantischen Naturbrücke (vgl. Grau 2019), führen diese Bestrebungen in unserer heutigen Zeit fort und manifestieren damit die denkmalpflegerische Zielsetzung, das Klassischen Erbe erhalten zu wollen. Ihren Wiederschein findet diese Fixierung in der wissenschaftlichen Auseinandersetzung (vgl. Müller-Wolff 2007), in der die Goethezeit in Bezug auf den Park überrepräsentiert, andere Phasen bislang jedoch völlig vernachlässigt wurden. Ein weiterer Grund, warum sich der Park in seiner einheitlichen Erscheinung erhalten hat, mag in der Schwierigkeit liegen, „dass sich die Garten und Parkanlagen des 18. und 19. Jahrhunderts der Neugestaltung widersetzten. Offensichtlich ist ihre Ästhetik so vollkommen, dass sie keine Verstöße gegen diese Gesetze zulässt" (Krause 2001: 158). Die immensen gartenpflegerischen
Bemühungen der Klassik Stiftung Weimar und ihre substantiell steigenden Bewirtschaftungskosten spiegeln insofern den Anspruch, „das universelle Erbe für die künftigen Generationen zu vermitteln und zu bewahren" (Grau et al. 2019). Mit Hilfe der Parkordnung, die unter anderem das Betreten weiter Teile der Parkwiesen untersagt, sind auch die Besucher*innen angehalten, durch ihr Verhalten einen Beitrag zum Erhalt des Parks zu leisten. Durch die touristisch in Szene gesetzten und museal bespielten Orte und Gebäude kann den Parkbesucher*innen dabei gezielt ein sprichwörtlich in Bahnen gelenktes Bild dieser Epoche vermittelt werden.

Im Hinblick auf seine heterotopischen Eigenschaften lässt sich somit zunächst konstatieren, dass die denkmalpflegerische Ausrichtung und Erhaltung des Parks aus ihrer Tradition heraus homogenisierend wirken. Dies verdeutlicht sich beispielsweise in der Rekonstruktion der bereits erwähnten romantischen Naturbrücke, die die aus dem ästhetischen Gesamtkonzept fallende Vorgängerbrücke ersetzt. Auch das Tempelherrenhaus, das in verschiedenen Epochen eine besondere Bedeutung für den Park hatte, fügt sich seit einem Bombentreffer im zweiten Weltkrieg als bewachsene Ruine in das romantische Ideal des englischen Landschaftsgartens ein (vgl. Eckardt et al. 2009: 52). Zwar lässt sich argumentieren, dass der Park in seiner denkmalpflegerischen Konzeption 
insofern heterotopische Qualitäten aufweist, dass er „selber außer der Zeit und sicher vor ihrem Zahn sein soll“" (Foucault 1992: 43), doch stehen diese Fixierung auf das klassische Erbe sowie die mit den denkmalpflegerischen Auflagen einhergehenden Einschränkungen und Verbote in vielerlei Hinsicht in Konflikt zu diesem Konzept. Die Gartengestaltung des Parks, die zu ihrer Zeit ägyptische, griechische, gotische und zeitgenössische Stilformen und Architekturen in einer neuen Ästhetik verband (vgl. Müller-Wolff 2007: 14) und damit sowohl Orte als auch Zeiten zusammenführte, „die eigentlich unvereinbar sind“ (Foucault 2013: 14), verengt sich aus heutiger Perspektive auf die etwa fünfzig Jahre, in denen dieser Ort von Carl August und Goethe gestaltet wurde (vgl. Huschke 1951: 115). Deutlich anerkannt werden muss, dass der Park als künstlerischer Ausdruck und Idealbild der Vereinigung von Natur und Kultur auch heute noch eine besondere Wirkung entfaltet. In diesem Sinne erfüllt er als Freizeit- und Erholungsraum kompensatorische und somit heterotopische gesellschaftliche Funktionen. Vollkommen in den Hintergrund treten darüber jedoch die konflikthafte Dimension und krisenhaften Ursprünge seiner Entstehung, wie sie sich aus der heterotopischen Perspektive für die Zeit der Weimarer Klassik eröffnet.

Nachfolgend soll daher aufgezeigt und vorgeschlagen werden, in welchen Formen heterotopische Eigenschaften und Funktionen dieses Ortes wieder stärker betont werden könnten. Zunächst wird der Blick zu diesem Zwecke auf eine historische Schicht des Parks gerichtet, die in der wissenschaftlichen Rezeption und in den übergreifenden Vermittlungskonzepten zum Park bislang gänzlich unberücksichtigt geblieben ist.

\section{Der Park als Austragungsort der Konflikte am Staatlichen Bauhaus}

Inwiefern lassen sich heterotopischen Eigenschaften des Parks in anderen Zeitschichten aufzeigen?

Eine räumlich-entwicklungsgeschichtliche Betrachtung des Zusammenhangs zwischen dem Park an der Ilm und dem Staatlichen Bauhaus Weimar hinsichtlich der historischen Aufarbeitung der Parkgeschichte und der Bauhaus-Forschung hat es bislang nicht gegeben. In diese Forschungslücke fiel der Befund eines 2017 durchgeführten urbanistischen Studienprojektes an der Bauhaus-Universität Weimar, das die Aufarbeitung der städtebaulichen Geschichte der Schulinstitution zum Thema hatte. Eine Betrachtung für den Zeitraum 1919 bis 1925 beobachtete hierbei eine signifikante Häufung von Aktivitäten, Aneignungen und Raumnutzungen im und rund um den Park 
an der Ilm durch die Protagonist*innen des Staatlichen Bauhaus Weimar (vgl. Werchohlad 2018a). Dennoch spielt die Zeit des historischen Bauhauses in Weimar in den einschlägigen Monografien zum Park kaum eine beziehungsweise keine Rolle (vgl. Huschke 1955; Müller-Wolff 2007). Die Gründe hierfür mögen der bereits erwähnten überragenden Präsenz des klassischen Erbes an diesem Ort, der geringen Bautätigkeit des Bauhauses in den Anfangsjahren (vgl. Hahn 2002: 272) oder auch der Tatsache geschuldet sein, dass beispielsweise das Haus am Horn als erster und einziger Wohnbau des Staatlichen Bauhaus in Weimar (vgl. bspw. Behr 1973) kurz hinter beziehungsweise an der heutigen Parkgrenze liegt. An diesem Punkt setzte daher eine Masterarbeit mit dem Titel „Die Natur des Bauhaus - der Park an der Ilm als Austragungsort des Konflikts am frühen Staatlichen Bauhaus" an (Werchohlad 2018b). Zusammenfassend dargestellt lassen die Ergebnisse der Masterarbeit den Schluss zu, dass der Park in der Zeit des historischen Bauhauses zur Bühne und zum umkämpften Austragungsort der gesellschaftspolitischen, stadtöffentlichen und schulinternen Konflikte und ihrer in Verbindung stehenden reformpädagogischen, lebensreformerischen und künstlerischen Ansätze und Konzepte wurde. Damit bot der Park ebenso Raum für eine, in der wissenschaftlichen Rezeption gleichsam noch deutlich unterrepräsentierte Seite des Bauhauses: die künstlerische Auseinandersetzung in und mit der Natur und die Suche nach Strategien des naturverbundenen Lebens und Gestaltens. Aktiv und konfliktreich setzte sich das Bauhaus dabei mit der in Weimar betriebenen Traditions- und Denkmalpflege, dem Akademismus und dem traditionellen Erbe auseinander (vgl. Bestgen 2009). Die Schule griff bewusst die in Weimar vorherrschenden Konventionen und Gepflogenheiten an, geriet darüber einerseits in den Fokus konservativer und rechtsnationaler Angriffe, konnte die hierdurch gesteigerte Aufmerksamkeit und Öffentlichkeit zeitweise jedoch ebenso selbstbestimmt und im eigenen Interesse nutzen (vgl. Kröll 1974: 11).

Vielfältige esoterische und naturverbundene Gruppierungen, etwa Mitglieder der Wandervogel-Bewegung (vgl. Schlemmer 1994c: 158) oder sektenartige Gemeinschaften, wie unter anderem eine sich um den Meister Johannes Itten versammelnde Mazdaznan-Glaubensgemeinschaft (vgl. Buch 1994), fanden im Park sowohl eine öffentliche Bühne als auch einen spirituellen Rückzugsraum. Itten selbst hatte im Park, genauer gesagt in dem aus der Goethezeit stammenden Tempelherrenhaus, sein Atelier bezogen, diese Nutzungsmöglichkeit laut seines Freundes Oskar Schlemmer (1994a: 456) sogar zur Bedingung seines Antritts gemacht. Atemübungen, Sonnenbäder, Fastenrituale (vgl. Citroen 1993: 64) und die auf Selbstversorgung 


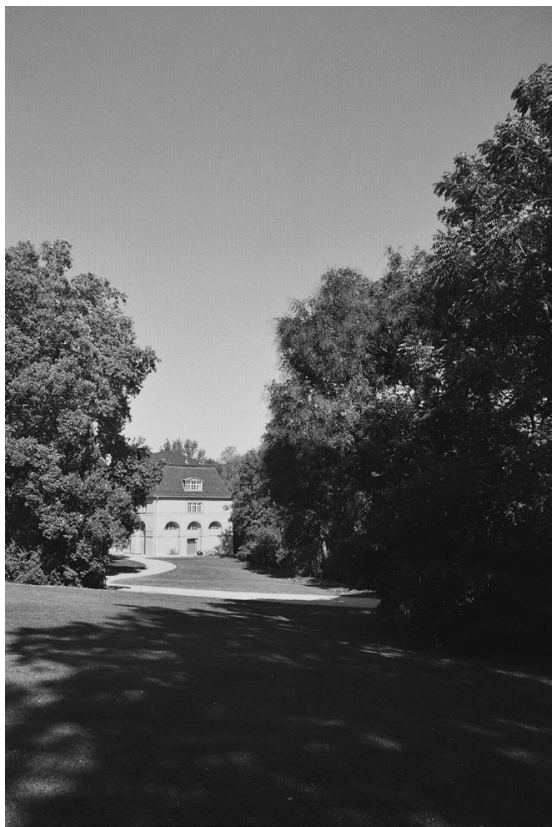

Abbildung 3: Das großherzogliche Reithaus an der Ilm: Hier sollte durch das Bauhaus eine „Probierwerkstatt“ zur fachübergreifenden Projektarbeit eingerichtet werden (Foto: Mats Werchohlad)

zielende Bewirtschaftung eines Ackers auf dem Gelände am Horn (vgl. Gropius 1987: 93) zeugen diesbezüglich von den vielfältigen naturverbundenen Ansätzen und Strategien, sich den Raum in diesem Sinne anzueignen. In der studentisch geleiteten Mensa wurden die selbst geernteten Erzeugnisse nach Rezepten der fernöstlichen Mazdaznan-Lehre zubereitet (vgl. Schlemmer 1994b: 458). Der Meister Lothar Schreyer, der in unmittelbarer Nachbarschaft zum Park im Dachboden des Hauses Charlotte von Steins wohnte, berichtet von einer abendlichen Unterhaltung, in der ihm ein Freund den Ratschlag gab: „Benutzen Sie stets den Weg durch den Park, wenn Sie ins Bauhaus gehen oder vom Bauhaus kommen, nicht die Straße. Im Park sind reinigende Kräfte“ (Schreyer 1956: 143f.). Der Park bot im Hinblick auf den Mystizismus und den Symbolismus der Anfangsjahre des Weimarer Bauhauses somit einen reichhaltigen ökologischen, historischen und diskursiven Resonanzraum. Nähert man sich dem historischen Bauhaus über diesen räumlich-historischen Zugang mit 
dem Park als Ausgangs- und Zugangsort, eröffnet sich ein symbolischer und mythischer Interpretationsraum, in dem sich abseits der festgeschriebenen historischen Narrative neue Verbindungslinien und Bezüge in der Auseinandersetzung und der Durchdringung von Natur und ihrer gesellschaftlichen Konstruktion offenbaren.

Die historische Aufbereitung kann somit verdeutlichen, dass sich der Park trotz oder gerade unter Einbeziehung des klassischen Erbes für neue Räume, Konzepte und Nutzungsformen öffnen lässt und diese integriert werden können. Manifest werden in der Betrachtung dieser Epoche die zahlreichen Konflikte, die mit den vielfältigen, alternativen und dem konservativen Geschmack zuwiderlaufenden Nutzungsformen im Park einhergehen. In dieser Phase treten insofern erneut und in besonderer Weise die heterotopischen
Eigenschaften des Parks hervor, durch die dieser Ort für das künstlerische Wirken am Staatlichen Bauhaus Weimar kaum zu unterschätzende räumlich-geistige Ressourcen schuf.

\section{Der Park als Spiegelraum einer konfliktreichen Gegenwart}

Durch welche Ansätze und Szenarien könnten diese heterotopischen Eigenschaften aufs Neue zur Entfaltung gebracht werden?

Unabhängig von dieser historischen Betrachtung, den wissenschaftlichen Strategien und Vermittlungsansätzen, die darauf abzielen, den Ort in seiner Ambivalenz und Vielschichtigkeit stärker hervorzuheben, trat der Park nun zuletzt von selbst immer stärker als Spiegelraum der ökologischen und damit einhergehenden

\section{9}

Nähert man sich dem historischen Bauhaus über diesen räumlich-historischen Zugang mit dem Park als Ausgangs- und Zugangsort, eröffnet sich ein symbolischer und mythischer Interpretationsraum, in dem sich abseits der festgeschriebenen historischen Narrative neue Verbindungslinien und Bezüge in der Auseinandersetzung und der Durchdringung von Natur und ihrer gesellschaftlichen Konstruktion offenbaren. 
gesellschaftlichen Herausforderungen und Konflikte hervor. Die Nähe zwischen Stadtund Parkraum (vgl. Günther et al. 1993: 344) verstärkt diese Tendenzen und macht sie besonders sichtbar. Dass damit schließlich der Erhalt des klassischen Erbes, des gartenkünstlerischen Gesamtkunstwerks in Gefahr geraten ist, stellt für Weimarer Verhältnisse eine Bedrohung dar, die kaum schwerer wiegen könnte. Bei der notwendigen Anpassung ihrer denkmalpflegerischen Ausrichtung steht die Stiftung insofern vor der Herausforderung, zukünftigen klimatischen Gegebenheiten sowie den neuen und gesteigerten Nutzungsansprüchen zu handeln. Fragen der politischen Teilhabe, Mitgestaltung und Vermittlung sind dabei zentral und müssen sensibel und wissensbasiert in offene und integrativ-wirkende Diskursräume überführt werden. Mit der Planung des Themenjahrs 2021, in dem zahlreiche Veranstaltungen, Interventionen und Vermittlungsangebote im Park umgesetzt werden sollen, schafft die Klassik Stiftung Weimar hierfür wichtige Voraussetzungen. Mit den verstärkten Aktivitäten, temporären Bauten und steigenden Besuchszahlen, die der Park während des Themenjahrs und im Rahmen der Bundesgartenschau erfahren wird, werden sich jedoch in gleichem Maße neue Fragen, Herausforderungen und Konflikte offenbaren.

Das Konzept der Heterotopie als Analysewerkzeug und Programm kann diesbezüglich darauf aufmerksam machen und daran erinnern, dass der Park an der Ilm in besonderer Weise die Qualitäten aufweist, diesen Konflikten und Herausforderungen einen Raum zu eröffnen. Darin ließe sich die Komplexität in ihren Ambivalenzen auf produktive, künstlerische und ästhetische Weise neu zusammenführen und vereinen. Der Klimawandel und die damit einhergehenden vielschichtigen gesellschaftlichen Umbrüche, in denen die „Menschen mit ihrer herkömmlichen Zeit brechen " (Foucault 1993: 43), verleiht der Suche nach solchen Orten eine enorme Dringlichkeit.

Mit diesem Programm stünde die Stiftung - als Institution - außerdem vor der herausragenden Aufgabe, ihr eigenes Selbstverständnis und damit die Mechanismen ihrer Reproduktion von Macht und Deutungshoheit zu hinterfragen. Denn durch ihre im Themenjahr verstärkte und sichtbarere Präsenz gerät die Stiftung wiederum in Gefahr, den Ort auf seine historischen Zuschreibungen und vorgegebenen Gestaltungsansätze zu reduzieren und zu fixieren. Um den Park in diesem Sinne zu einem wirklichen Gegenraum zu machen, einem Ort, der sich „allen anderen widersetzen“ (Foucault 2013: 10) kann, steht die Stiftung somit in der Verantwortung, vielfältige Nutzungsgruppen mit einzubeziehen und umgekehrt, Räume für unvorhergesehene Nutzungen zu öffnen. Für diesen Prozess ist 
die Stiftung gleichsam auf Hilfe von außen angewiesen. Mit der im Sommersemester 2020 an der Bauhaus-Universität Weimar durchgeführten öffentlichen Vortrags- und Diskussionsreihe Heterotopie Ilmpark wurden hierfür zahlreiche Anknüpfungspunkte geschaffen. In dieser brachten dreizehn Professuren aus verschiedenen Fachbereichen, Studierende der Universität und Mitarbeitende der Stiftung ihre Perspektiven und Positionen zu diesem Ort in Austausch. Dem Deutungs- und Wahrnehmungsraum des Parks konnten in diesem Rahmen geologische, denkmalpflegerische, künstlerische, sozialwissenschaftliche, architekturtheoretische, medienwissenschaftliche und weitere Schichten hinzugefügt werden. Aufzeichnungen, Protokolle, Videos und entwickelte Positionen zum Zustand und zur Zukunft des Parks wurden dazu auf einem Blog gesammelt, um kontinuierlich erweitert und für die Stiftung zur Disposition gestellt zu werden. In diesem Sinne schafft das Projekt zahlreiche Anknüpfungspunkte, die sich in fortlaufenden Kooperationen und Projekten im Themenjahr aufgreifen und weiterführen ließen.

\section{Der Park als Ausgangspunkt für die Suche nach einer neuen Umweltästhetik}

Übergreifend konnten die verschiedenen Perspektiven und Beiträge der Vortragsreihe in ihrer Vielfältigkeit und Bandbreite verdeutlichen, dass der Park, in seinen heterotopischen Eigenschaften, einen besonderen Rahmen schafft, um Lehrinhalte und wissenschaftliche Positionen im Spannungsfeld von Umwelt und Gesellschaft in konkreter wie abstrakter Weise neu zusammenzuführen und zu verorten. Bereits 2018 hatte die Leiterin der Gartenabteilung, Catrin Seidel diesbezüglich gefordert:

Langfristige Strategien brauchen als Grundlage eine interdisziplinäre, wissenschaftliche Erforschung unserer Gartenkunstwerke. (Grau et al. 2018)

Die Vortragsreihe und das Themenjahr könnten in diesem Sinne als Plattform zur Entwicklung eines fachübergreifenden Studienprogramms genutzt werden, das den Park an der Ilm zum Forschungs-, Erlebnis- und Verhandlungsraum für die Suche nach einer neuen Umweltästhetik macht.

Die zentralen Aspekte und die Notwendigkeit einer solchen Umweltästhetik sind durch den Naturphilosoph Gernot Böhme schon früh umrissen und als Gast der Vortragsreihe in einem live übertragenem Vortrag und Gespräch im Römischen Haus noch einmal für den spezifischen Ort aktualisiert vorgebracht und diskutiert worden. Nach Böhme (vgl. 1994: 84) müsse eine solche Umweltästhetik die dichotomen Kategorienbildung von Natur und Technik überwinden. In ihr äußere sich ein Bewusstsein des Selbst als unauflöslicher Teil der 
Natur, das in der Lage ist, Gestaltungsstrategien in und mit der Natur zu entwerfen (vgl. Böhme 1994: 84). Zentral steht dabei die Erkenntnis, dass Natur heutzutage immer zugleich sozial konstituierte Natur meint (vgl. Böhme 1989: 94). Der Park als künstlich gestaltete, gleichsam natürlich wirkende Landschaft steht hierfür paradigmatisch (vgl. Böhme 1989: 92). Insofern zielt die Suche nach einer neuen Umweltästhetik auf eine Vorstellung von Natur, die die Grenzen des Parks weit hinter sich lässt und alle Bereiche und Formen einer menschlich durchdrungenen Umwelt, inklusive der neuen Medien mit einschließt (vgl. Böhme 2013: 11). Dieses neue Naturverständnis offenbart sich für Böhme im Zwischenbereich von Kunst und Wissenschaft:

Es ist deshalb für die eigentliche Wissenschaft bereits ein Stück Phantasie und Spekulation notwendig. Das Wissen über die Natur auszusprechen heißt so bereits produktiv über die Natur hinauszugehen, heißt Kunst. (Böhme 1989: 101)

Die Vereinigung dieser scheinbar unauflöslichen Wechselbeziehung sei als ästhetisches Erlebnis im Medium der Atmosphäre zu erreichen (vgl. Böhme 2013: 15). Insofern liegt in der Gestaltung von geistig, körperlich und emotional erfahrbaren Atmosphären eine wesentliche Voraussetzung und somit notwenige Kompetenz bei der Suche nach einer neuen Umweltästhetik (vgl. Böhme 2013: 15).
Gerade im Park lässt sich das szenische Arrangement solcher, mannigfaltiger Atmosphären nachvollziehen. Böhme führt aus, dass sich die dem Park zugrunde liegende Gartentheorie Cay Lorenz Hirschfelds (1990) als quasi-objektiver Katalog lese, durch den sich die verschiedenen gewünschten Stimmungen und Atmosphären gartenkünstlerisch erzeugen und kompositorisch arrangieren ließen (vgl. Böhme 1989: 90).

Im Hinblick auf die Zukunft dieses Ortes stellt sich somit die Frage, ob sich im Park neue, andere und zusätzliche Räume eröffnen und integrieren lassen, in denen sich die wachsenden Widersprüche, klimatischen Phänomene und steigenden Komplexitäten ebenfalls atmosphärisch vermitteln, erleben und ergründen lassen. Ein Studienprogramm könnte sich diese Aufgabe zum Ziel setzen und den Ort dafür als Ausgangspukt nutzen. Im Park ließen sich die darin entwickelten Fragestellungen, Ansätze und Ideen durch seine heterotopischen Qualitäten, in räumlicher Hinsicht zur Entfaltung bringen. Historische, ökologische, biologische, technische und ästhetische Lehrinhalte könnten so mit praktischen Lehrkonzepten zur Körperlichkeit und Wahrnehmungsschulung in neue Zusammenhänge gebracht werden. Der Park würde auf diese Weise zum Ort, um historische Lehrkonzepte und zeitgenössische Fragestellungen in Verbindung zu setzen, noch unbekannte Räume und Atmosphären $\mathrm{zu}$ schaffen und darüber zu einer neuen Umweltästhetik zu gelangen. 


\section{LITERATUR}

Behr, Adalbert (1973): Das Musterhaus des Staatlichen Bauhauses in Weimar von 1923. In: Wissenschaftliche Zeitschrift der Hochschule für Architektur und Bauwesen 20, S. 167-177.

Berger, Joachim (2001): Carl August als Bauherr und Bewohner des Römischen Hauses. In: Beyer, Andreas (Hrsg.): Das Römische Haus in Weimar. München: Hanser, S. 25-39.

Bestgen, Ulrike (2009): Wie kann Weimar zu neuer Blüte gelangen?: ,Gropius the Romantic' und der vermeintliche Kampf gegen die klassische Weltanschauung. In: Ackermann, Ute/Bestgen, Ulrike/Klassik Stiftung Weimar (Hrsg.): Das Bauhaus kommt aus Weimar. Berlin/ Weimar: Klassik Stiftung Weimar, S. 19-23.

Beyer, Andreas (2001): Die Restaurierung des Römischen Hauses 1996-1999. In: Beyer, Andreas (Hrsg.): Das Römische Haus in Weimar. München: Hanser, S. 132-147.

Böhme, Gernot (1989): Für eine ökologische Naturästhetik. Frankfurt am Main: Suhrkamp.

Böhme, Gernot (2013): Atmosphäre. Essays zur neuen Ästhetik. Berlin: Suhrkamp.

Busch, Ludger (1994): Das Bauhaus und Mazdaznan. In: Bothe, Rolf/Hahn, Peter (Hrsg.): Das frühe Bauhaus und Johannes Itten: Katalogbuch anläßlich des 75. Gründungsjubiläums des Staatlichen Bauhauses in Weimar. Weimar/ Bern/ Berlin: Gerd Hatje, S. 83-90.

Busch-Salmen, Gabriele/Salmen, Walter/Michel, Christoph (Hrsg.) (1998): Der Weimarer Musenhof: Dichtung, Musikund Tanz, Gartenkunst, Geselligkeit, Malerei. Stuttgart: J.B. Metzler.

Dietzsch, Folke F. (1990): Die Studierenden am Bauhaus: eine analytische Betrachtung zur strukturellen Zusammensetzung der Studierenden, zu ihrem Studium und Leben am Bauhaus sowie zu ihrem späteren Wirken. Weimar: Hochschule für Architektur und Bauwesen.

Döring, Jörg/Thielmann, Tristan (Hrsg.) (2009): Spatial Turn: das Raumparadigma in den Kultur- und Sozialwissenschaften. Bielefeld: transcript.

Eckardt, Michael/Rohde, Theres Sophie/Bauhaus-Universität Weimar (Hrsg.) (2009): Bauhaus-Spaziergang: in Weimar unterwegs auf den Spuren des frühen Bauhauses. Weimar: Verlag der Bauhaus-Universität.
Foucault, Michel (1992): Andere Räume. In: Barck, Karlheinz/Gente, Peter/Paris, Heidi (Hrsg.): Aisthesis. Wahrnehmung heute oder Perspektiven einer anderen Ästhetik. Leipzig: Reclam, S. 34-46.

Foucault, Michel (2013) Die Heterotopien. Der utopische Körper. Zwei Radiovorträge. Berlin: Suhrkamp.

Grau, Benjamin (2018): Trockenheit gefährdet Baumbestand. In: „Blog der Klassik Stiftung Weimar“, 06.07.2018. Online verfügbar unter https://blog.klassik-stiftung.de/ trockenheit-gefaehrdet-baumbestand (14.01.2020).

Grau, Benjamin (2020): Neubau der Naturbrücke im Park an der Ilm. „Blog der Klassik Stiftung Weimar“, 05.12.2019. Online verfügbar unter https://blog.klassik-stiftung.de/ neubau-der-naturbruecke/ (31.08.2020).

Grau, Benjamin/Lentz, Christian/Seidl, Catrin (2018): Die Weimarer Parks leiden unter Wetterextremen. In: „Blog der Klassik Stiftung Weimar“, 07.12.2018. Online verfügbar unter https://blog.klassik-stiftung.de/weimarer-parks-leiden-unter-wetterextremen (14.01.2020).

Grober, Ulrich (2010): Die Entdeckung der Nachhaltigkeit: Kulturgeschichte eines Begriffs. München: Kunstmann.

Gropius, Walter (1987 [1923]): Idee und Aufbau des Staatlichen Bauhauses Weimar. In: Probst, Hartmut/Schädlich, Christian/Hochschule für Architektur und Bauwesen Weimar (Hrsg.): Walter Gropius - Ausgewählte Schriften. Band Teil 3. Berlin: Verlag für Bauwesen, S. 71-72.

Günther, Gitta/Huschke, Wolfram/Steiner, Walter (Hrsg.) (1993): Weimar: Lexikon zur Stadtgeschichte. Weimar: Böhlau.

Günzel, Stephan/Kümmerling, Franziska (Hrsg.) (2010): Raum: Ein interdisziplinäres Handbuch. Stuttgart: Metzler.

Hahn, Peter (2002): Kampf der Geister Itten und Gropius am frühen Bauhaus. In: Denaro, Dolores (Hrsg.): Johannes Itten Wege zur Kunst. Ostfildern-Ruit: Hatje, S. 256-273.

Hirschfeld, Christian Cay Lorenz (1990): Theorie der Gartenkunst. Berlin: Union.

Huschke, Wolfgang (1951): Die Geschichte des Parkes von Weimar. Weimar: Böhlau.

Huschke, Wolfgang (1955): Park um Weimar: ein Buch von Dichtung und Gartenkunst. Weimar: Hermann Böhlaus. 
Knoche, Michael (2007): Die Weimarer Bibliothek als Büchersammlung, Museum und Erinnerungsort. In: Seemann, Hellmut (Hrsg.): Anna Amalia, Carl August und das Ereignis Weimar. Göttingen: Wallstein.

Krause, Karoline (2001): Ein klassischer Park für das Volk - Rezeption der Gartenkunst und Gartendenkmalpflege am Beispiel des Parks an der Ilm. In: Ehrlich, Lothar/Mai, Gunther (Hrsg.): Weimarer Klassik in der Ära Honecker. Weimar: Böhlau.

Kropmanns, Peter (2020): Kulturgüter oder Liegewiesen: Wie die Pariser ihre Parks kaputtmachen. In: „Frankfurter Allgemeine Zeitung“, 27.07.2020. Online verfügbar unter https://www.faz.net/1.6877071 (30.08.2020).

Kurianowicz, Tomasz (2020): „Corona, what?! Im Park wird um die Freiheit gekämpft. In: „Berliner Zeitung“11.08.2020. Online verfügbar unter https://www.berliner-zeitung.de/ kultur-vergnuegen/coronaparty-berghain-corona-coronaleugner-park-protest-hasenheide-gleisdreieckpark-protest-jugendkultur-elektro-dj-berghain-im-park-wird-um-die-freiheit-gekaempft-li.97394 (30.08.2020).

Merseburger, Peter (1999): Mythos Weimar: Zwischen Geist und Macht. Stuttgart: DVA.

Müller, Gerhard (2007): Goethe und Carl August Freundschaft und Politik. In: Seemann, Hellmut (Hrsg.): Anna Amalia, Carl August und das Ereignis Weimar. Göttingen: Wallstein.

Müller, Ulrich (2001): „Ziehet das Genie des Orts zu Rathe; [...] es mahlet, indem ihr pflanzet, und zeichnet Entwürfe, indem ihr anleget. “In: Beyer, Andreas (Hrsg.): Das Römische Haus in Weimar. München: Hanser.

Müller-Wolff, Susanne (2007): Ein Landschaftsgarten im Ilmtal: die Geschichte des herzoglichen Parks in Weimar. Köln: Böhlau.

Rohde, Michael/Krellig, Heiner (2014): Historische Gärten im Klimawandel: Empfehlungen zur Bewahrung. Leipzig: Edition Leipzig.

Ruoff, Michael (2013): Foucault-Lexikon: Entwicklung Kernbegriffe - Zusammenhänge. Paderborn: Wilhelm Fink.

Schlemmer, Oskar (1994a [1920]): Brief an Otto Meyer-Amden, 7. August 1920. In: Bothe, Rolf/Hahn, Peter (Hrsg.) Das frühe Bauhaus und Johannes Itten: Katalogbuch anläßlich des 75. Gründungsjubiläums des Staatlichen Bauhauses in Weimar. Weimar/ Bern/ Berlin: Gerd Hatje, S. 456.
Schlemmer, Oskar (1994b [1921a]): Brief an Otto Meyer-Amden Weimar, 14. Juli 1921. In: Bothe, Rolf/ Hahn, Peter (Hrsg.): Das frühe Bauhaus und Johannes Itten: Katalogbuch anläßlich des 75. Gründungsjubiläums des Staatlichen Bauhauses in Weimar. Weimar/ Bern/ Berlin: Gerd Hatje, S. 458.

Schlemmer, Oskar (1994c [1921b]): Brief an Otto Meyer-Amden vom 7. Dezember 1921. In: Bothe, Rolf/ Hahn, Peter (Hrsg.): Das frühe Bauhaus und Johannes Itten: Katalogbuch anläßlich des 75. Gründungsjubiläums des Staatlichen Bauhauses in Weimar. Weimar/ Bern/ Berlin: Gerd Hatje, S. 458.

Schroer, Markus (2006): Räume, Orte, Grenzen: auf dem Weg zu einer Soziologie des Raums. Frankfurt am Main: Suhrkamp.

Seidl, Catrin/Ertl, Theresa (2019): Zwischen Überschwemmungen und Dürren: Prof. Dr. Norbert Kühn im Interview über Auswirkungen des Klimawandels in historischen Gärten. In: „Blog der Klassik Stiftung Weimar“, 23.05.1919. Online verfügbar unter https://blog.klassik-stiftung.de/zwischen-ueberschwemmungen-und-duerren/ (14.01.2020).

Soja, Edward W. (1989): Postmodern geographies: The reassertion of space in critical social theory. London/ New York: Verso.

Soja, Edward W. (2009): Vom ,Zeitgeist' zum ,Raumgeist‘. New Twists on the Spatial Turn“. In: Döring, Jörg/Thielmann, Tristan (Hrsg.): Spatial Turn: das Raumparadigma in den Kultur- und Sozialwissenschaften. Bielefeld: transcript, S. 241-263.

UNESCO (1998): Übersetzung der Erklärung zum außergewöhnlichen universellen Wert - Klassisches Weimar. In: Auswärtiges Amt. Online verfügbar unter https:// www.auswaertiges-amt.de/de/aussenpolitik/internationale-organisationen/welterbestaetten/212964 (01.08.2020).

Van Zantwijk, Temilo (2007): Das Ereignis Weimar-Jena als Symbol einer geistigen Welt. In: Seemann, Hellmut (Hrsg.): Anna Amalia, Carl August und das Ereignis Weimar. Göttingen: Wallstein, S. 118-132.

Warning, Rainer (2015): Utopie und Heterotopie. In: Dünne, Jörg/Mahler, Andreas (Hrsg.): Handbuch Literatur \& Raum. Berlin/ Boston: De Gruyter.

Weber, Christiane (2020): Vandalismus im Weimarer Ilmpark hat deutlich zugenommen. In: „Thüringer Allgemeine“, 06.08.2020. Online verfügbar unter https://www.thueringer-allgemeine.de/regionen/weimar/vandalismus-hat-im-ilmpark-deutlich-zugenommen-id230112640.html (30.08.2020). 
Werchohlad, Mats (2018a): Die Natur des Bauhaus. In: Welch Guerra, Max (Projektleiter) (Hrsg.): Die Bauhaus-Universität Weimar und die politische Geschichte hinter ihrem Städtebau : Forschungsprojekt : Professur Raumplanung und Raumforschung, Institut für Europäische Urbanistik, Bauhaus-Universität Weimar. (unveröffentlicht).

Werchohlad, Mats (2018b): Die Natur des Bauhaus Der Park an der Ilm als Austragungsort des Konflikts am Staatlichen Bauhaus. Professur Raumplanung und Raumforschung, Institut für Europäische Urbanistik, Bauhaus-Universität Weimar. (unveröffentlicht).

\section{ZUM AUTOR}

Mats Werchohlad, geboren 1990, studierte im Bachelor Maschinenbau an der TU Berlin, machte anknüpfend daran einen Master im Fach Urbanistik an der Bauhaus-Universität Weimar und belegte zusätzliche Kurse in Medienwissenschaften.
Seitdem erkundet er interdisziplinäre $\mathrm{Zu}$ gänge im Schnittfeld von Technik, Bildung und Raum an Orten wie Smart Cities oder englischen Landschaftsgärten. Seit seinem Abschluss arbeitet er an der Professur für Raumplanung und Raumforschung und aktuell an der Entwicklung eines fachübergreifenden Studiengangs im Verständnis der Liberal Arts and Sciences.

An dem Beitrag haben folgende Redaktionsmitglieder im Review, Betreuung und Lektorat mitgearbeitet: Veronika Riedl, Tamara Schwertel, Andreas Schulz, Tonka Radisch und Nils Haacke.

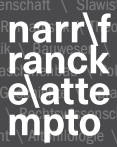

\section{BUCHTIPP}

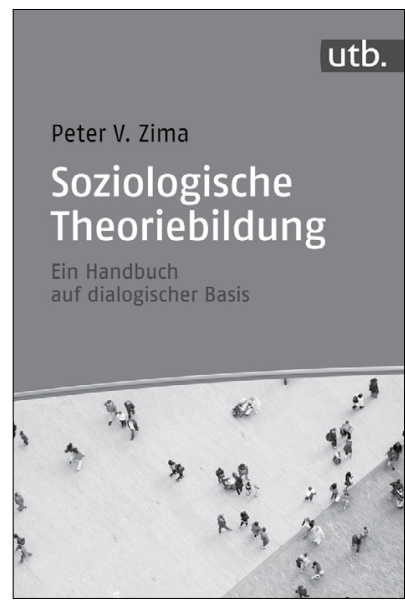

Peter V. Zima

\section{Soziologische Theoriebildung}

Ein Handbuch auf dialogischer Basis

2020, 1090 Seiten

$€[D] 34,99$

ISBN 978-3-8252-5370-7

eISBN 978-3-8385-5370-2

Dieses Handbuch bietet eine Übersicht über die soziologische Theoriebildung der letzten 200 Jahre. Beginnend mit Hegel, Marx und Comte, werden zusammen mit den Klassikern (Durkheim, M. Weber, Parsons) und der Spätmoderne (Luhmann, Habermas, Touraine) die wichtigsten soziologischen Theorien bis hin zur Postmoderne (Baudrillard, Sennett, Foucault) behandelt. Dabei wird jeweils auch der historischphilosophische Kontext rekonstruiert. Anders als die meisten Einführungen in die soziologische Theorie hat dieser Band eine dialogische Struktur: Der Bezug der Theorien aufeinander lässt ihre Stärken, Schwächen und Besonderheiten hervortreten. Im metatheoretischen Dialog werden die kommentierten Theorien getestet. Sie werden konstruktivistisch als Erzählstrukturen aufgefasst, die aus besonderen, kontingenten Perspektiven hervorgehen. Die Kapitel verknüpfen jeweils vier Ebenen miteinander: Dialogizität, Narrativität, Terminologie und Historizität. Der Band wendet sich an fortgeschrittene Studierende und eignet sich hervorragend als Begleiter für das ganze Studium der Soziologie. 


\section{Literatur zum Thema}

\section{Buchempfehlungen der Redaktion}

von Luisa Bischoff

$\begin{aligned} & \text { Die soziale } \\ & \text { Die soziale } \\ & \text { Konstitution } \\ & \text { der Umwelt }\end{aligned} \begin{aligned} & \text { Konstitution } \\ & \text { der Umwelt. } \\ & \text { von Klaus Kraemer } \\ & \text { vS Verlag für Sozialwissen- } \\ & \text { schaften } 2008 \\ & \text { ISBN: } 9783531158303\end{aligned}$
$49,99 €$

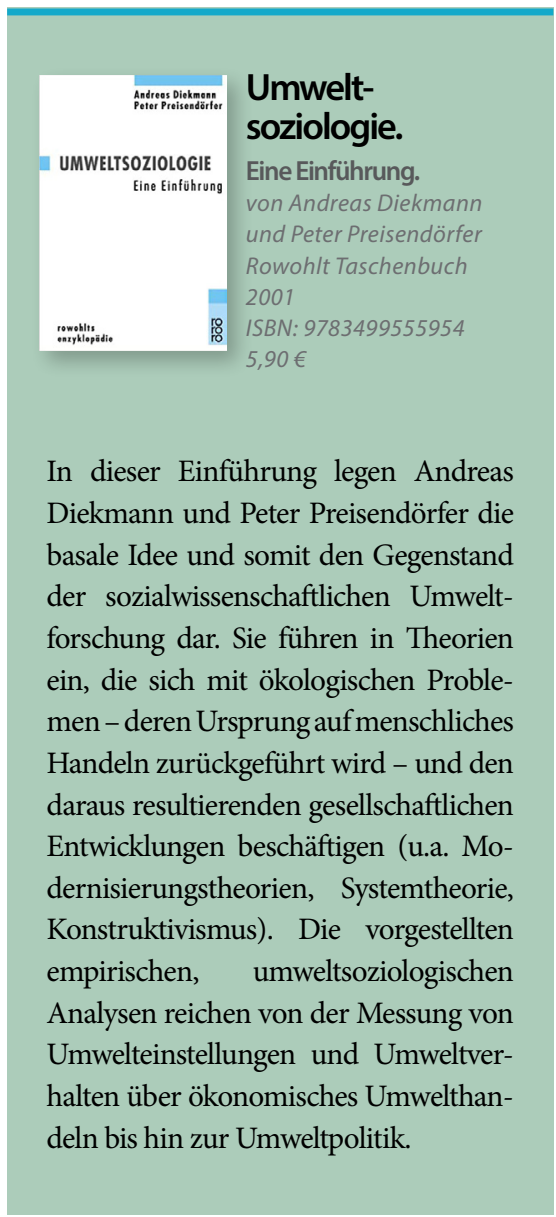




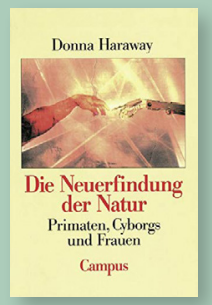

\section{Die Neu- erfindung der Natur.}

Primaten, Cyborgs und Frauen.

von Donna Haraway

Campus 1995

ISBN: 9783593352411

$21,50 €$
Diese Essaysammlung spiegelt entlang von zentralen Begriffen das theoretische Denken der feministischen Naturwissenschaftlerin und Wissenschaftshistorikerin Donna Haraway wider, von Cyborgs, über situiertes Wissen bis zu Primaten. Dabei „entzaubert“ sie Wissenschaftsdiskurse und -praktiken, indem sie nicht nur hegemoniale Dualismen wie MannFrau oder Mensch-Tier dekonstruiert, sondern auch die Auflösung der Natur selbst imaginiert. Sie zeigt auf, wie nicht nur die Grenzziehung zwischen Mensch und Tier mit dem Aufkommen der Evolutionstheorie fluide wurde, sondern auch, wie zunehmend Grenzen zwischen beispielsweise Mensch und Maschine - ergo zwischen Natur und Technik verschwimmen. Haraway fasst Technik jedoch mehr als Möglichkeit denn als Bedrohung auf und plädiert für eine neue soziale Praxis, die die Grenzziehungen zwischen Natur und Kultur sowie die damit einhergehenden Herrschaftsverhältnisse nachhaltig destabilisiert.

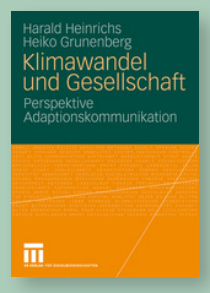

\section{Klimawandel und Gesellschaft.}

Perspektive Adaptationskommunikation.

von Harald Heinrichs und

Heiko Grunenberg

VS Verlag für Sozialwissenschaften 2009

ISBN: 9783531915166 $54,99 €$

Die Autoren fragen in diesem Buch nach den regionalen Auswirkungen und Reaktionen des Klimawandels in Deutschland. Sie fokussieren auf emergierende Kommunikations- und Partizipationsprozesse, die für eine gesellschaftliche Antwort auf den Klimawandel notwendig werden und entwickeln ein theoretisches Konzept der Adaptionskommunikation. Anhand einer Fallstudie zu Hochwasser in Bremen und Hamburg, zeigen die Autoren auf, wie Betroffene nicht nur kommunikativ, sondern auch partizipativ in solche Prozesse eingebunden werden können. Indem dargelegt wird, wo bezüglich der gesellschaftlichen Klimawandel-Adaption in Deutschland noch Forschungslücken sind und wie diese Adaption praktisch gestaltet werden kann, ist das Buch nicht nur für Umweltsoziolog*innen relevant, sondern für alle, die sich aktiv und lokal gegen den Klimawandel einbringen wollen. 


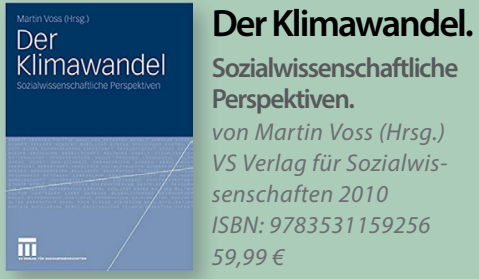

Im Gegensatzzur Umweltsoziologiesteckt die sozialwissenschaftliche Forschung zum Klimawandel und zur Anpassung an ihn noch in den Kinderschuhen. Wie vielfältig sozialwissenschaftliche Klimaforschung ist, wird an der interdisziplinären Ausrichtung des Sammelbandes deutlich: Es wird nicht nur eine transdisziplinäre Diskussion geführt, sondern auch die Frage gestellt, welche Implikationen der Klimawandel für die Sozialforschung hat. Gleichzeitig finden sich in dem Sammelband beispielsweise eine Diskursanalyse zu Eisbären und Eisbergen, eine Diskussion zur „Ethik in Zeiten des Klimawandels“ ebenso wie Analysen zu psychologischen Folgen, zu Lebensstilen, zu Gouvernementalität, zur (In) Stabilität durch Massenkommunikation, zur Klimagerechtigkeit oder zur Rolle von Religiosität. Der Sammelband bietet einen aktuellen Einblick in die Klimawandelforschung und schließt mit einem möglichen Forschungsprogramm für künftige sozialwissenschaftliche Auseinandersetzungen mit dem Klimawandel.

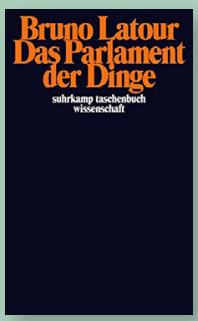

\section{Das Parlament der Dinge.}

Für eine politische

Ökologie.

von Bruno Latour

Suhrkamp 2010 [2001]

ISBN: 9783518295540

$20,00 €$

Mit diesem Essay legte der Philosoph und Soziologe Bruno Latour den Grundstein für seine politische Ökologie, die er aktuell in "Kampf um Gaia. Acht Vorträge über das neue Klimaregime“ (2017) und „Das terrestrische Manifest" (2018) weiterdenkt. Er beginnt damit, den Begriff Natur radikal in Frage zu stellen und fordert ein alternatives Verständnis, das Natur in einem wissenschaftlich-konzipierenden Prozess der Wahrheitsfindung verortet und nicht als präsoziale Gegebenheit fasst. Dies impliziert, dass der Natur kein Sosein, keine Essenz zu Grunde liegt, sondern dass sie neu gedacht werden kann: in Latours Denken als „politische Epistemologie“. Darunter versteht er exemplarisch am Beispiel der (globalen) grünen Politik, dass diese eine Kommunikation - gar ein Kollektiv - zwischen menschlichen und nichtmenschlichen (aber ebenfalls sozialisierten) Wesen anstreben solle, um nicht länger in unproduktivem Ökologie-Politik- bzw. Natur-KulturDenken zu verharren. 


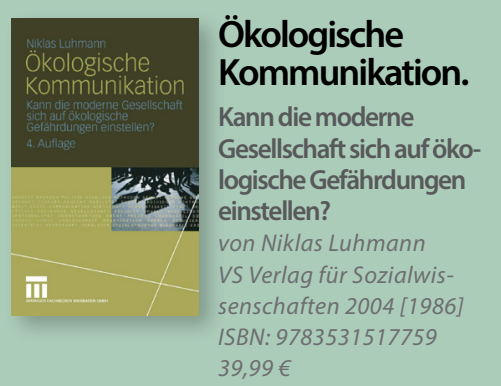

Niklas Luhmann setzt sich in diesem Buch zur ökologischen Kommunikation mit den gesellschaftlichen (Un)Möglichkeiten der Reaktionen auf Umweltprobleme auseinander. In seiner Arbeit beschreibt er, wie die Eigenlogik gesellschaftlich basaler Funktionssysteme - wie die der Politik, Wirtschaft, Wissenschaft oder Religion - ökologische Kommunikation beschränkt und angemessene Strategien konterkariert. Diese Kommunikationsschwierigkeit versteht er vor dem Hintergrund, dass viele Umweltprobleme menschengemacht sind, als umso schwerwiegender. Selbst Versuche des politisch-initiierten Klimaschutzes oder Protestformen gegen die ökologische Kommunikation der einzelnen Systeme bleibt der Eigenlogik der Systeme und damit den kommunikativen Beschränkungen verhaftet. Als mögliche Lösung identifiziert Luhmann dabei eine ausgewogene Resonanz zwischen den Systemen und ist damit nicht nur für gegenwärtige Umwelt- bzw. Klimawandelbewegungen, sondern auch in Hinblick auf Harmut Rosas Resonanz-Theorie anschlussfähig.

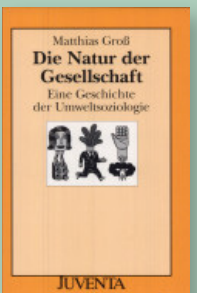

\section{Die Natur der Gesellschaft.}

Eine Geschichte der Umweltsoziologie.

von Matthias Groß

Juventa 2001

ISBN: 9783779910718 $35,00 €$

Matthias Groß, einer der führenden Umweltsoziolog ${ }^{\star}$ innen im deutschsprachigen Raum, zeichnet in seiner Dissertation wissenschaftstheoretisch nach, wie sich die anfängliche Umweltsoziologie aus der europäischen (Marx, Durkheim, Weber) und der amerikanischen (u.a. Ross, Giddings) Tradition formierte. An diese soziologischen Klassiker kaum Anschluss nehmend entstanden in den 1920er Jahren humanökologische und regionalsoziologische Verknüpfungen von Stadt, Land und Gesellschaft (v.a. Park und Simmel). Abschließend bespricht Groß die sich seit den 1970er Jahren herausbildenden (sozial)konstruktivistischen Umwelt-Gesellschafts-Konzeptionen und zeigt damit nicht nur auf, wie Umweltprobleme wissenschaftshistorisch eingeordnet wurden und werden, sondern auch, wie sich die daran anschließenden gesellschaftlichen Diskurse historisch verschieben.

Die Buchempfehlungen wurden von Luisa Bischoff erstellt und von Andreas Schulz und Tonka Radisch lektoriert. 


\section{MACH MIT!}

\section{... im Redaktionsteam}

Eine E-Mail genügt! Wir suchen stets neue Gesichter mit frischen Ideen. Aktuell brauchen wir Unterstützung insbesondere für das Lektorat, den Satz, die Autor*innenbetreuung sowie für die Durchführung von Interviews für unseren YouTube-Channel.

Wenn ihr nicht zum ständigen Redaktionsteam gehören wollt, gibt es die Möglichkeit, uns im Rahmen des Kuratoriums (bzw. Freundinnenkreises) mit Rat und Tat zur Seite zu stehen.

\section{... als Autor*in in unserem Magazin}

Schickt uns zu unserem aktuellen Call4Papers eure wissenschatlichen Artikel. Außerdem nehmen wir in unseren Serviceteil „Perspektiven” gerne auch Rezensionen, Tagungsberichte, Interviews oder andere soziologische Inputs mit auf.

\section{... als Blogger*in}

Schickt uns eure Ideen für Rezensionen aktueller soziologischer Bücher, eigene soziologische Blog-Beiträge oder Interview-Vorschläge an: redaktion[at]soziologiemagazin.de

Meldet euch bei uns oder leitet einen Hinweis auf uns in eurem soziologisch interessierten Umfeld weiter. Wir freuen uns! 


\section{Tagungen und Termine}

(1) Die ökologische Frage: Herausforderungen für die soziologische Theorie

Die Tagung der DGS-Sektion Soziologische Theorie findet am 21. und 22. Januar 2021 in Frankfurt am Main statt.

2 Medien - Demokratie - Bildung: Normative Vermittlungsprozesse und Diversität in mediatisierten Gesellschaften

Das Interdisciplinary Media Ethics Center der TU Dortmund veranstaltet diese Tagung vom 20. bis zum 22. Januar 2021 in Dortmund.

3 Figuren und Perspektiven auf die Masse und das Individuum in der kapitalistischen Moderne (XIX-XXI Jahrhundert)

Die interdisziplinäre Tagung des „Jungen Forums“ findet am 26. und 27. Februar 2021 am Zentrum Marc Bloch in Berlin statt.

4 Die Grundlagen der Menschenrechte: moralisch, politisch oder sozial?

Die Tagung der Sektion Politische Theorie und Ideengeschichte der Deutschen Vereinigung für Politikwissenschaft findet am 11. und 12. März 2021 an der TU Dresden statt.

5 Revolution der Paarbeziehungen? Der Wandel des Beziehungslebens in Bundesrepublik und DDR

Die Tagung findet am Leibniz-Zentrum für Zeithistorische Forschung in Potsdam am 11. und 12. März 2021 statt.

6 Spring School Wissenssoziologische Diskursanalyse

Vom 15. bis zum 17. März 2021 findet die Spring School an der Universität Augsburg statt.

7 Die diskursive Konstruktion von Wirklichkeit V - Interdisziplinäre Perspektiven einer wissenssoziologischen Diskursforschung

Die Tagung findet am 18. und 19. März 2021 an der Universität Augsburg statt.

8 Theorien in der qualitativen Forschung

Das Institut für Publizistik- und Kommunikationswissenschaft veranstaltet die 3. Tagung des Netzwerks Qualitative Methoden, die vom 30. März bis zum 1. April 2021 an der Freien Universität Berlin stattfindet. 


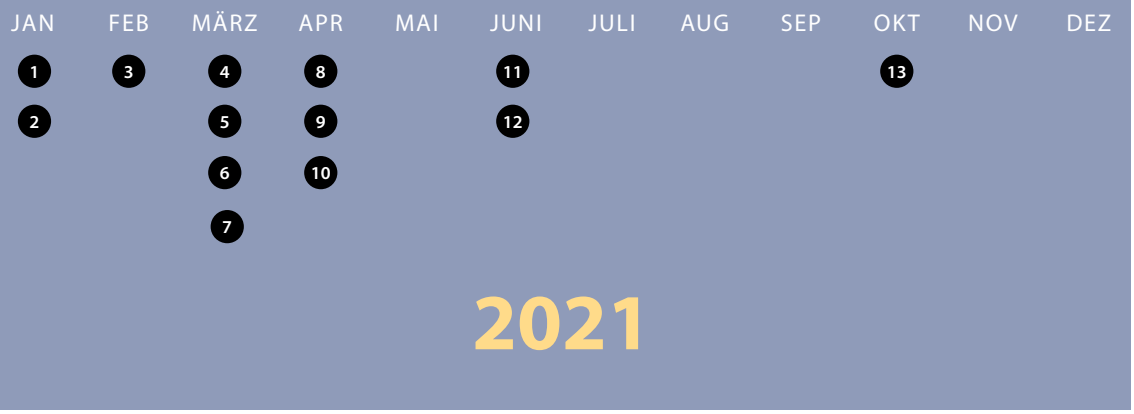

9 Territorien, Staat und Nation in der Wirtschafts- und Sozialgeschichte

Die Gesellschaft für Sozial- und Wirtschaftsgeschichte (GSWG) und der Wirtschaftshistorische Ausschuss des Vereins für Socialpolitik (VfS) veranstalten gemeinsam ihren 4. Kongress für Wirtschafts- und Sozialgeschichte vom 7. bis zum 9. April 2021 in Wien.

10 \#Kommunikation \#(R)Evolution. Zum Wandel der Kommunikation in der digitalen Gesellschaft

Die Dreiländertagung für Kommunikationswissenschaft findet vom 7. bis zum 9. April 2021 online mit punktuellen Präsenz-Elementen in Zürich statt.

\section{(11) Rethinking Transparancy: Challenging Ideals and Embracing Paradoxes}

Der dritte Global Summit 2020 wurde verlegt und findet vom 18. bis zum 20. Juni 2021 an der Freien Universität Berlin statt.

(12 Radical Health. Doing Medicine, Health Care, and Anthropology of the Good

Die Konferenz der Arbeitsgemeinschaft Medical Anthropology der Deutschen Gesellschaft für Sozial- und Kulturanthropologie und der Arbeitsgemeinschaft Ethnologie und Medizin (AGEM) wurde verschoben und findet vom 25. bis zum 27. Juni 2021 an der Freien Universität Berlin statt.

13 Vielfältige Familien: Elternschaft und Familie/n jenseits von Heteronormativität und Zweigeschlechtlichkeit

Das Zentrum für transdisziplinäre Geschlechterstudien der Humboldt-Universität Berlin veranstaltet diese Konferenz am 7. und 8. Oktober 2021 in Berlin.

Die Terminübersicht 2021 wurde von Tonka Radisch recherchiert und von Andreas Schulz lektoriert. 


\section{Redaktionsteam}

Andreas Schulz (MA, M.A.), hat u.a. Soziologie sowie Publizistik- und Kommunikationswissenschaft in Leipzig, Bern und Wien studiert. Aufgaben: Vereinsvorstand, Heft- und Lektoratskoordination, Review, Autor*innenbetreuung und Lektorat.

Cathrin Mund (M.A.), promoviert am Lehrstuhl für Kultur- und Religionssoziologie an der Universität Bayreuth. Aufgaben:Vorstandsmitglied, Heft- und Lektoratskoordination, Review, Autor*innenbetreuung, Betreuung Wissenschaftlicher Beirat und Lektorat.

Daniel Bräunling (B.A.), studiert Soziologie in Tübingen. Aufgaben: Review und Autor*innenbetreuung.

Hendrik Erz (M.A.), hat Geschichte, Politikwissenschaften und Soziologie in Bonn studiert. Derzeit ist er Doktorand am Institut für Analytische Soziologie (IAS) an der Universität Linköping, Schweden. Aufgaben: Vorstandsmitglied, Social Media, Blog.

Luisa Bischoff (M.A.), arbeitet im Graduiertenkolleg,Doing Transitions' in Frankfurt/ Main. Aufgaben: Lektorat.

Markus Kohlmeier (M.A.), promoviert an der Universität Duisburg-Essen. Aufgaben: Vorstandsmitglied, Anzeigen, Review und Autor*innenbetreuung.

Marlene Müller-Brandeck (M.A.), hat Soziologie und Philosophie an der LudwigMaximilians-Universität München studiert. Aufgaben: Layout, Satz und Bildredaktion.

Nils Haacke (M.A.), hat Soziologie an der Albert-Ludwigs-Universität Freiburg studiert. Aufgabe: Review und Autor*innenbetreuung.

Tamara Schwertel (M.A.), studierte Soziologie mit Schwerpunkt empirische Methoden der Sozialforschung sowie Philosophie in Frankfurt/Main. Aufgaben: Stellvertretender Vereinsvorstand und Review.

Tanja Strukelj (B.A.), studiert Soziologie in Frankfurt/Main. Aufgaben:Vorstandsmitglied, Social Media und Lektorat.

Tonka Radisch, studiert Soziologie in Frankfurt/Main. Aufgaben: Lektorat.

Veronika Riedl (B.A.), studiert Soziologie an der Universität Innsbruck. Aufgaben: Review, Autor*innenbetreuung und Satz. 


\section{DANKSAGUNG}

Das Soziologiemagazin wird - samt dem dazugehörigen Verein - ausschließlich von ehrenamtlich arbeitenden Menschen getragen: Studierende und Absolvent*innen der Soziologie und/oder verwandter Fächer, aber auch Promovierende sowie den wissenschaftlichen Mitarbeiter*innen und Professor*innen, die sich bei uns als wissenschaftliche Beiräte engagieren. An all diejenigen möchten wir auch diesmal ein herzliches und großes Dankeschön aussprechen. Danke für Eure und Ihre Energie, für die investierte Zeit und Mühe, für Diskussionen und Absprachen sowohl in der Redaktion als auch mit den Autor*innen. Ein solches Engagement ist nicht selbstverständlich und soll deshalb an dieser Stelle dezidiert bedacht, genannt und gewürdigt werden! Des Weiteren durften wir uns auch diesmal mit zahlreichen und diversen Beiträgen auseinandersetzen; vielen Dank an die dazugehörigen Autor*innen, die Lust, Zeit und vielleicht in manchen Fällen auch Mut gefunden haben, ihre Artikel einzusenden und sich dem Review-Verfahren zu stellen. Ohne solche Einsendungen und Rückmeldungen wäre unsere Arbeit frustrierend oder sogar schlicht unmöglich. Außerdem bedanken wir uns beim Verlag Barbara Budrich für die produktive und zuverlässige Zusammenarbeit. So, und das letzte große Dankeschön geht an die Leser*innen unserer Magazine und des Blogs und an die Menschen, die uns auf Facebook, Twitter und YouTube folgen. Aufgrund Eurer starken Unterstützung macht es uns wiederum großen Spaß, das Magazin - mit allem, was dazu gehört - auf die Beine zu stellen und damit auch weiterhin eine Publikationsplattform für Studierende und Promovierende der Sozialwissenschaften zu bieten. 


\section{$M \underset{\text { The Art of Data nalysis }}{\operatorname{MaX}}$}

Allround-Software für Datenbearbeitung, Visualisierung, Analyse und Berichterstellung

MAXQDA ist ein leistungsfähiges Analysetool für zahlreiche Datenarten und vielfältige methodische Ansätze wie Qualitative Inhaltsanalyse, Mixed-Methods-Analyse, Grounded Theory, Online Research Methods und viele mehr.

Für Bildungseinrichtungen \& Studierende bieten wir spezielle Lizenzen an: maxqda.de/lizenzen

MAXQDA jetzt testen: maxqda.de/demo 


\section{Impressum}

\author{
HERAUSGEBER \\ soziologiemagazin e.V. \\ Ludwig-Maximilians-Universität München \\ Institut für Soziologie \\ Konradstraße 6 \\ 80801 München \\ RECHTSSITZ: Halle (Saale) \\ VEREINSVORSTAND (VISDPR) \\ Andreas Schulz (Vorsitzender) \\ Tamara Schwertel (stellv. Vorsitzende) \\ Markus Kohlmeier (Finanzen) \\ Tanja Strukelj \\ Hendrik Erz \\ Cathrin Mund \\ vorstand@soziologiemagazin.de
}

\section{REDAKTION}

Andreas Schulz, Cathrin Mund, Daniel Bräunling, Franziska Deutschmann, Frederic Markus Gerdon, Hendrik Erz, Leonard Mach, Lucas Steger, Luisa Bischoff, Markus Kohlmeier, Marlene Müller-Brandeck, Nils Haacke, Tamara Schwertel, Tanja Strukelj, Tonka Radisch, Veronika Riedl

\section{FRAGEN BITTE AN}

redaktion@soziologiemagazin.de

LAYOUT UND SATZ: Marlene Müller-Brandeck,

Veronika Riedl

TITELBILD: Marlene Müller-Brandeck

\section{ANZEIGEN}

Ansprechpartner: Markus Kohlmeier

anzeigen@soziologiemagazin.de

Es gilt die Anzeigenpreisliste vom 01.05.2015

\section{WISSENSCHAFTLICHER BEIRAT}

Prof. Dr. Clemens Albrecht, Prof. Dr. Brigitte Aulenbacher, Prof. Dr. Birgit Blättel-Mink, Prof. Dr. Ulrich Bröckling, Prof. Dr. Aldo Haesler, Prof. Dr. Ernst von Kardorff, Prof. Dr. Hubert Knoblauch, Prof. Dr. em. Reinhard Kreckel, Prof. Dr. Thomas Kron, Dr. Diana Lindner, Prof. Dr. Kurt Mühler, Dr. Yvonne Niekrenz, Dipl. Sozialwirt Harald Ritzau, Dr. Cornelia Schadler, Dr. Imke Schmincke, Dr. Jasmin Siri, Dr. Irene Somm, Prof. Dr. Manfred Stock, Dr. Sylvia Terpe, apl. Prof. Dr. Udo Thiedeke, Prof. Dr. Georg Vobruba, Dr. Greta Wagner
ERSCHEINEN UND BEZUGSBEDINGUNGEN

Jährlich zwei Hefte. Open Access

PREIS: Einzelheft Print EUR 13,00;

Abonnement Print: EUR 22,00/ Jahr, Abonnement Print ermäßigt EUR 18,00/ Jahr (inkl. MwSt., zzgl. Versandkosten);

E-JOURNAL: kostenlos

Das digitale Angebot finden Sie auf:

sozmag.budrich-journals.de und auf

www.soziologiemagazin.de

\section{BESTELLUNGEN PRINT}

bitte an den Buchhandel oder den

Verlag Barbara Budrich

Stauffenbergstr. 7

D-51379 Leverkusen-Opladen

Tel.: +49 (0)2171.344.594

Fax: +49 (0)2171.344.693

info@budrich.de

www.budrich.de

www.budrich-journals.de

www.shop.budrich.de

Heft 22, Jg. 13, 2020

(c) 2020 Verlag Barbara Budrich GmbH

Opladen | Berlin | Toronto

ISSN 2198-980X

(C) 2020 Dieses Werk ist bei der Verlag Barbara Budrich $\mathrm{GmbH}$ erschienen und steht unter der Creative Commons Lizenz Attribution-ShareAlike 4.0 International (CC BY-SA 4.0): https://creativecommons.org/licenses/by-sa/4.0/.

Diese Lizenz erlaubt die Verbreitung, Speicherung, Vervielfältigung und Bearbeitung bei Verwendung der gleichen CC-BY-SA 4.0-Lizenz und unter Angabe der Urheberlnnen, Rechte, Änderungen und verwendeten Lizenz.

Dieses Heft steht im Open-Access-Bereich der Verlagsseite zum kostenlosen Download bereit (https:// doi.org/10.3224/soz.v13i2.00).

Eine kostenpflichtige Druckversion (Print on Demand) kann über den Verlag bezogen werden.

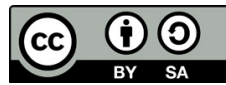




\section{SOZIOLOGIE}

\section{Nichts als die Wahrheit? Verschwörungsglaube und konspirationistisches Denken}

Ob auf Hygiene-Demos oder den Social-Media-Accounts von Prominenten - sogenannte Verschwörungstheorien haben derzeit Konjunktur. Insbesondere während der CoronaPandemie rückten sie wieder zunehmend in den Fokus der öffentlichen wie medialen Aufmerksamkeit. Aber auch schon vor der Pandemie waren diverse Verschwörungsmythen im Umlauf: Bevor sich Thesen um eine CoronaVerschwörung und die damit angestrebte Neuordnung der Welt verbreiteten, erhielten insbesondere Mythen rechter Akteur*innen Aufwind. Sie warnten in Bezug auf Flucht- und Migrationsbewegungen vor dem "Großen Austausch“, die sogenannte „Reichsbürgerbewegung " zweifelte die politische Handlungsfähigkeit Deutschlands an und es gab Akteur*innen, die den Klimawandel für frei erfunden hielten.

Trotz der jüngsten Aufmerksamkeit ist konspirationistisches Denken kein Phänomen des 21. Jahrhunderts: Der Historiker Dieter Groh (1992: 267ff.) bezeichnet es gar als „anthropologische Konstante“, da Verschwörungstheorien $\mathrm{zu}$ allen Zeiten und in allen Gesellschaften vorkämen. Seit dem Mittelalter zirkulieren zahlreiche antisemitische Verschwörungsmythen und im 18. und 19. Jahrhundert entstanden erste Mythen rund um Freimaurerbünde und Illuminaten sowie deren Weltherrschaftsstreben.

Bei Verschwörungsmythen muss es sich, wie vielfach angenommen, nicht allein um randständige Theorien handeln, die jenseits eingeschworener Gruppen abgelehnt werden: Die kollektive Praxis der Hexen- und Jüd*innenverfolgungen zeigt, dass Verschwörungsideologien Eingang in hegemoniale Diskurse finden und zu herrschenden Wahrheiten aufstreben können. Seine Manifestation findet konspirationistisches Denken nicht zuletzt in Formen der ,post-truth politics, wie etwa die Politologen Joseph Uscinski (2019) und Eiríkur Bergmann (2018) zeigen.

Versteht man Verschwörungsmythen als eine spezielle Form sozialen Wissens, stellt sich die Frage nach ihrer gesellschaftlichen Funktion. Die Wissenssoziologen Andreas Anton und Kollegen gehen davon aus, dass es durch Verschwörungstheorien möglich werde, „Ereignisse oder Prozesse, die sich ansonsten nur schwer einordnen ließen, sinnhaft zu deuten“" (Anton et al. 2014: 15). Interessant ist aber auch die Frage danach, wie sich die Mehrheitsgesellschaft mit heterodoxen Verschwörungstheorien auseinandersetzt: Wirkt das Label als „Verschwörungstheorie“ möglicherweise sogar stigmatisierend, um einem Hegemonieverlust entgegenzuwirken? (vgl. u. a. Knobloch 2018)

Was hat es mit Verschwörungsideologien und konspirationistischem Denken auf sich? Worauf reagieren Menschen, die Verschwörungsmythen in die Welt setzen und welche sozialen Funktionen kommen diesen Mythen zu? Wie werden Verschwörungsmythen verbreitet - und wie rezipiert? Welche Akteur*innen sind beteiligt? Und inwiefern (be)dienen solche Mythen Populist*innen? Zeitdiagnosen und Auseinandersetzungen mit aktuellen Verschwörungstheorien, historischsoziologische Perspektiven, aber auch die Auseinandersetzung mit spezifischen Gruppen wie der „Reichsbürgerbewegung“ oder den Corona-Leugner*innen können interessante Einsichten in aktuelle Diskurse und liefern.

Wir suchen eure Perspektiven, Analysen und Gedanken! Sendet eure Texte zum Thema bis zum 1. Dezember 2020 an einsendungen[at]soziologiemagazin.de.

Referenzen abrufbar unter:www.soziologiemagazin.de 